PFC/RR-94-6

\title{
Current Distribution in Cable-in-Conduit Conductors
}

\author{
Matthew A. Ferri
}

May 1994

\author{
Plasma Fusion Center \\ Massachusetts Institute of Techology \\ Cambridge, Massachusetts 02139
}

\section{DISCLAIMER}

\begin{abstract}
This report was prepared as an account of work sponsored by an agency of the United States Government. Neither the United States Government nor any agency thereof, nor any of their employees, makes any warranty, express or implied, or assumes any legal liability or responsibility for the accuracy, completeness, or usefulness of any information, apparatus, product, or process disclosed, or represents that its use would not infringe privately owned rights. Reference herein to any specific commercial product, process, or service by trade name, trademark, manufacturer, or otherwise does not necessarily constitute or imply its endorsement, recommendation, or favoring by the United States Government or any agency thereof. The views and opinions of authors expressed herein do not necessarily state or reflect those of the United States Government or any agency thereof.
\end{abstract}

This research was performed under appointment to the Magnetic Fusion Energy Technology Fellowship Program administered by Oak Ridge Institute for Science and Education for the U.S. Department of Energy.

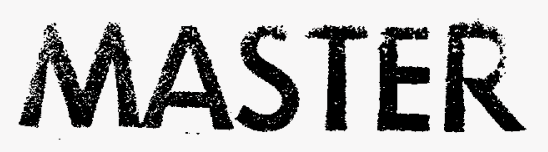


(c) 1994 Matthew A. Ferri. All rights reserved.

The author hereby grants to MIT permission to reproduce and to distribute publicly paper and electronic copies of this thesis document in whole or in part.

The Goverment reserves for itself and others acting on its behalf a royalty free, nonexclusive, irrevocable, world-wide license for governmental purposes to publish, distribute, translate, duplicate, exhibit, and perform any such data copyrighted by the contractor. 


\section{DISCLAIMER}

Portions of this document may be illegible in electronic image products. Images are produced from the best available original document. 


\title{
Current Distribution in Cable-in-Conduit Superconductors
}

by

\author{
Matthew A. Ferri
}

Submitted to the Department of Nuclear Engineering and the Department of Electrical Engineering and Computer Science on May 6, 1994, in partial fulfillment of the requirements for the Degrees of Master of Science in Nuclear Engineering and Master of Science in Electrical Engineering and Computer Science

\begin{abstract}
A numerical study of the current distribution in Cable-In-Conduit Conductors (CICC's) experiencing linearly ramping transport currents and transverse magnetic fields was conducted for both infinitely long, periodic cables and finite length cables terminated in low resistance joints. The goal of the study was to gain insight into the phenomenon known as Ramp Rate Limitation, an as yet unexplained correspondence between maximum attainable current and the ramp time taken to reach that current in CICC superconducting magnets. A discrete geometric model of a 27 strand multiply twisted CICC was developed to effectively represent the flux linkages, mutual inductances, and resistive contact points between the strands of an experimentally tested cable.
\end{abstract}

The results of the numerical study showed that for fully periodic cables, the current imbalances due to ramping magnetic fields and ramping transport currents are negligible in the range of experimentally explored operating conditions. For finite length, joint terminated cables, however, significant imbalances can exist. Unfortunately, quantitative results are limited by a lack of knowledge of the transverse resistance between strands in the joints. Nonetheless, general results are presented showing the dependency of the imbalance on cable length, ramp time, and joint resistance for both ramping transverse magnet fields and ramping transport currents.

At the conclusion of the study, it is suggested that calculated current imbalances in a finite length cable could cause certain strands to prematurely "quench"-become non-superconducting--thus leading to an instability for the entire cable. This numerically predicted "current imbalance instability" is compared to the experimentally observed Ramp Rate Limitation for the 27 strand CICC sample. Using the unknown quantity of the transverse joint resistance as the single fitting parameter, the results of the hypothesis reasonably match several major trends in the data. It is concluded that the results are encouraging but require further refinement and a direct measurement of the transverse joint resistance.

Thesis Supervisor: Dr. Joseph V. Minervini

Title: Acting Head of the Fusion Technology and Engineering Division MIT Plasma Fusion Center 


\section{Acknowledgments}

I would like to thank my advisors, Dr. Joseph Minervini, Professor Jeffrey Freidberg and Dr. Stephen Umans, for their help and encouragement in preparing this thesis. I would also like to thank my colleagues in the Superconducting Magnet Development Group at the MIT Plasma Fusion Center for their many contributions to this thesis, especially Drs. Chen-Yu Gung, Makoto Takayasu, and Vytaly Vysotsky. Finally, I would like to thank my fellowship sponsor for making my studies at MIT possible.

This research was performed under appointment to the Magnetic Fusion Energy Technology Fellowship Program administered by Oak Ridge Institute for Science and Education for the U.S. Department of Energy. 


\section{Table of Contents}

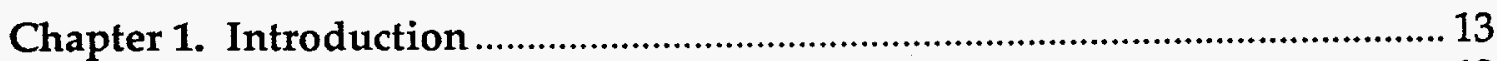

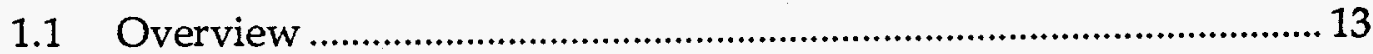

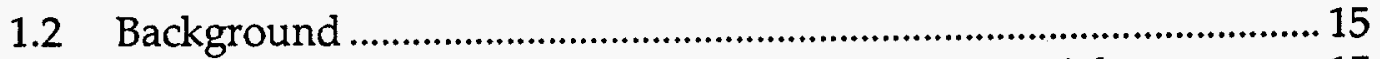

1.2.1 Basic Properties of Superconducting Materials ................... 15

1.2.2 Cable-in-Conduit Conductors ............................................... 18

1.2.3 Ramp Rate Limitation............................................................. 22

Chapter 2. Current Distribution in Linearly Ramped Cable-in-Conduit

Superconducting Magnets..................................................... 27

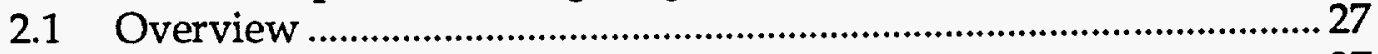

2.1.1 The Experimental Magnets ....................................................... 27

2.1.2 Causes of Uneven Current Distribution .................................29

Uneven Joint Resistances ........................................................ 29

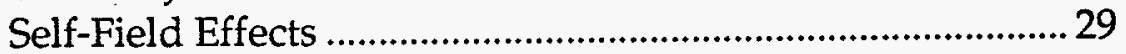

External-Field Effects ................................................................. 30

2.2 Approach to Studying Current Distribution...................................... 30

2.2.1 Properties of the 27 Strand Experimental Cable.................. 31

2.2.2 Model Assumptions ..................................................................32

2.2.3 Discretized Cable Geometry ………….................................... 33

The Maps: ...................................................................................34

A Comparison between "Pure Twisting" and

"Discretized" Models: ................................................. 38

2.2.4 Circuit Description from Maps ............................................ 41

Parallel and Transverse Currents: ........................................... 41

Inductive Elements: ................................................................ 42

Resistive Elements:......................................................................4 43

Flux Linkages: .........................................................................45

Governing Equations: .................................................................46

2.3 Current Distribution in CICC for Several Scenarios ............................46

Self-Field Cases:......................................................................... 47

External-Field Cases: ..................................................................... 47

2.3.1 Current Distribution in Infinitely Long, Periodic

CICC ...............................................................................4

2.3.1.1 Self-Field, Time-Dependent Solution: ......................48

Mathematical Model: ....................................................... 49

Results: ...........................................................................5 53

2.3.1.2 External-Field, Time-Dependent Solution:............56

Mathematical Model: ......................................................5 57

Results:....................................................................... 58

2.3.1.3 External-Field, Steady-State Solution:....................61

Mathematical Model:.........................................................61

Results: ...........................................................................6 65 


\subsubsection{Current Distribution in Finite Length, Joint}

Terminated CICC .............................................................68

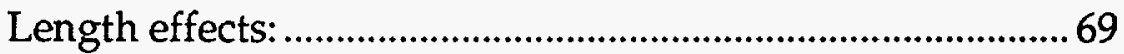

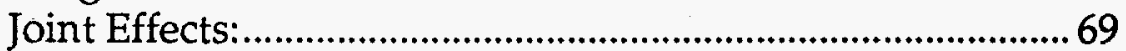

Time scales: ............................................................................... 70

2.3.2.1 Self-Field, Steady-State Solution: ............................. 71

Mathematical Model: ......................................................71

Results: .......................................................................... 73

2.3.2.2 External-Field, Time-Dependent Solution:............76 76

Mathematical Model: ....................................................77

Results: ................................................................

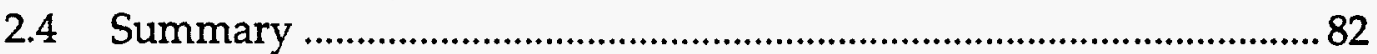

Infinite, Periodic Cases: ........................................................ 82

Finite, Joint Terminated Cases: ............................................83

Chapter 3. Ramp Rate Stability in CICC Magnets .............................................. 85

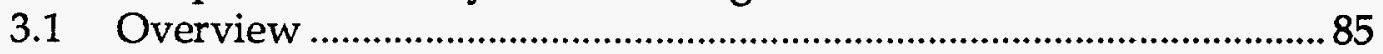

3.2 Simplified Stability Model Based on Current Distribution for

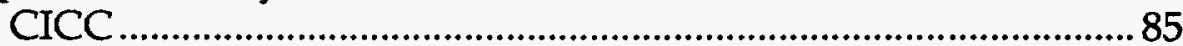

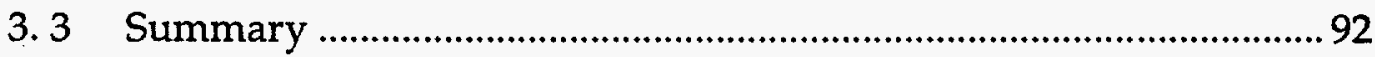

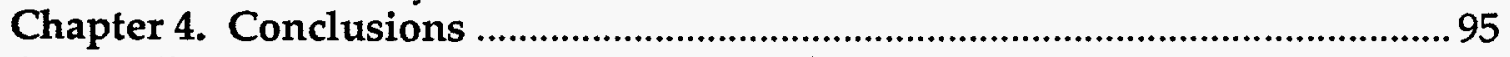

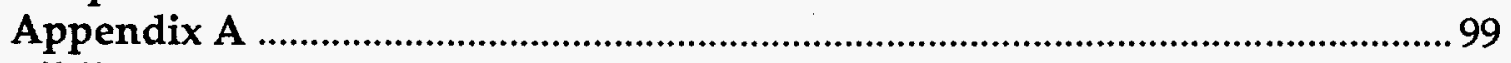

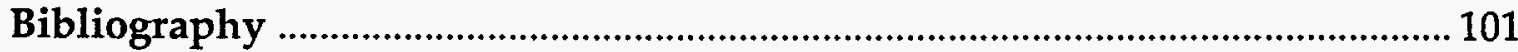




\section{List of Figures}

1.1 An example of a Cable-in-Conduit Conductor (CICC) shown in cross-section. Typically, the strands are twisted into triplets which are further twisted into bundles which are then twisted into subcables and so on until the entire cable is formed. Each strand consists of numerous superconducting filaments (not shown in this figure). (Hoenig [1980])

1.2 The critical surface plot for a commercially available $\mathrm{Nb}$-Ti alloy. At points below the surface, the alloy is superconducting, above the surface, it is normal. Source: reprinted with permission from Wilson, Superconducting Magnets, Copyright $\odot 1983$ by Oxford University Press.

1.3 A "simply twisted" cable. Strands on the outside remain on the outside over the length of the cable. Strands near the center stay near the center. (Hoenig, et al., [1975]).

1.4 A "fully transposed" cable in the form of a multiply twisted "rope." Source: reprinted with permission from Wilson, Superconducting Magnets, Copyright $(1) 1983$ by Oxford University Press.

1.5 Quench current vs. ramp time for the US-DPC experiment. The time developments of the current and magnetic field are shown (inset). The degraded performance at faster ramp times is known as the "Ramp Rate Limitation." (Painter, et al., [1992])

2.1 Schematic of the 27 strand US-DPC simulation experiment. A single loop coil formed from the CICC was placed in the bore of a $12 \mathrm{~T}$ Bitter magnet. The ratio of background field to transport current was kept constant as they were both linearly ramped in time.

2.2 The first 8 strand configurations of the 48 segment period of the 27 strand cable model. See text (Section 2.2.3) for explanation. .35

2.3 Between each segment of the cable, the strands rotate according to their prescribed twist pitches. Each triplet (heavy, solid triangle) rotates $52.5^{\circ}$. Each bundle (heavy, dotted triangles) rotates $22.5^{\circ}$, and the cable (lighter, dashed triangle) rotates $7.5^{\circ}$. See text (Section 2.2.3) for explanation.

2.4 The geometry of a simplified representation of the 27 strand cable is shown. The position of each strand in polar coordinates at each axial position $\mathrm{z}$ is $(\mathrm{r}(\mathrm{z}), \mathrm{q}(\mathrm{z}))$ and is a summation of the three rotating vectors which define the individual twist pitches of each stage of the cable, as shown. 
2.5 A comparison of the trajectory of strand 1 for two different geometric models of a 27 strand CICC: a) the discrete model used in calculations, b) a simpler, continuous model.

2.6 A section of the "Mesh Network Circuit" formed by three twisted strands with intermittent contacts. (The first strand is repeated at the bottom of the to facilitate the drawing.) The parallel and transverse currents (I 's and i's, respectively) are shown as they are defined in the text. The flux linkage areas ( $\left.\mathrm{A}^{\prime} \mathrm{s}\right)$ are shown as if the strands were co-planar--the effects of twisting are difficult to show, but must be included in the solution. The resistors all have a value of R1 and the inductors all have self-inductance L1. The mutual inductances (not shown) must be calculated from the geometry.

2.7 A schematic of the transverse resistance measurement used to establish the value of R1 used in numerical analyses. See text for details.

2.8 Normalized current vs. normalized axial position at several dimensionless times for a periodic 27 strand cable experiencing a linearly ramping transport current. The imbalances are caused by the self-field effect. The current distributions for strands 1, 2, and 3 are shown. The distributions for other strands can be related to 1 , 2 , or $3 u$ sing Table 2. For the experimental parameters, time $t=t^{\prime}$ (sec).

2.9 Normalized current vs. normalized axial position at several dimensionless times for a periodic 27 strand cable experiencing a linearly ramping external magnetic field. The current distributions for strands 1, 2, and 3 are shown. For the experimental parameters, time $\mathrm{t}=\mathrm{t}^{\prime}(\mathrm{sec})$

2.10 Normalized current vs. normalized axial position as time approaches infinity for a periodic 27 strand cable experiencing a linearly ramping external magnetic field. The current distributions for strands 1 through 9 are shown. The distributions for other strands can be related to 1 through 9 using Table 3 .

2.11 The transverse currents between strands 1 and 2 can be solved indepently from other currents in the cable. The necessary KVL equations are indicated by "loops" in the figure. Periodic boundary conditions are used to give an equal number of equations as unknowns.

2.12 Normalized current vs. normalized axial position in the steady state limit for a periodic 27 strand cable experiencing a linearly ramping external magnetic field. The current distributions for strands 1 through 9 are shown. The distributions for other strands can be related to 1 through 9 using Table 3 . 
2.13 Comparison of the direct steady state solution and the asymptotically approached limits of the transient analysis for a ramping external field.

2.14 Maximum induced current in the cable vs. "doubling factor" for ramping external fields. See text for explanation.

2.15 The self-field effect for linear ramping transport current in a finite length CICC can be approximated using a model composed of mutually coupled inductors joined in parallel.

2.16 Current Imbalances vs. Normalized length of cable for linearly ramping transport currents. See text for details.

2.17 Current Imbalances vs. multiple of final twist pitch length for linearly ramping transport currents using a simplified geometric model. The twist pitches of each stage of cable are equal to their nominal values. The results should be compared to Figure 2.18.

2.18 Current Imbalances vs. multiple of final twist pitch length for linearly ramping transport currents using a simplified geometric model. The twist pitches are 2-\% longer than the nominal value for the triplets, $10 \%$ longer for the bundles, and $5 \%$ longer for the final stage The results should be compared to Figure 2.17.

2.19 Idealized model of a four strand cable terminated in low resistance joints. Each strand is connected to the others at each joint through a resistance, $\mathrm{Rj}$. The strands are inductively coupled over their lengths but can otherwise be considered insulated. See text for further details.

2.20 Dimensionless current vs. dimensionless time for the strand experiencing the maximum induced current due to a linearly ramping external magnetic field for cables of four dimensionless lengths: 24, 72, 120, and 1224 segment lengths. Each current approaches the same asymptotic value, but the time constant of each curve is length dependent.

2.21 The maximum induced current in the cable is length dependent. At lengths approaching an integer multiple of the period length (48 segment lengths), the induced currents are minimal. At integerplus-half multiples of the period length, the induced currents are maximized.

2.22 Histogram of the steady state currents induced by a linearly ramping magnetic field for each strand in the 27 strand sample for a cable length equal to an integer-plus-half multiple of the period length.

3.1 Histogram of the current imbalance due to a linearly ramping transport current for each strand in the 27 strand sample for a cable length equal to the length of the actual experimental cable, $0.84 \mathrm{~m}$ 
(200 segment lengths). Strands with a normalized current equal to unity are carrying $1 / 27$ th of the transport current.

3.2 The maximum induced current in the 27 strand sample due to a linearly ramping external magnetic field vs. time. Both current and time are dimensionless. The total sample length is $0.84 \mathrm{~m}(200$ segment lengths) with $0.30 \mathrm{~m}$ (72 segment lengths) experiencing a transverse magnetic field. The dimensionless time constant of the exponential time-development is found to be tau $=2.0$, giving the dashed-line curve-fit shown.

3.3 Measured data from Ramp-Rate Limitation tests on a 27 strand CICC. Solid circles $(\bullet)$ represent points at which the cable quenched during a linear ramp to the quench current in time, tramp. Open circles $\left(^{\circ}\right)$ represent currents which were reached without quench for ramp time, tramp. (Takayasu, et al., [1992]) 


\section{Chapter 1}

Introduction

\subsection{Overview}

Next generation fusion devices such as ITER and TPX are relying on superconducting magnets consisting of Cable-In-Conduit Conductors (CICC) to provide the "magnetic bottle" needed for plasma confinement. A CICC superconductor is composed of multiple strands of conductor, each of which is composed of numerous superconducting filaments embedded in a nonsuperconducting matrix, typically made of copper. The strands are twisted together and compacted within a structural conduit which provides a passage for a liquid helium coolant. A cross-section of a typical CICC is diagrammed in Figure 1.1.

Recently, lower than expected limiting currents have been encountered in CICC superconducting magnets when the current is ramped at a constant rate. It has been suggested that this "Ramp Rate Limitation" may be due to an uneven distribution of current amongst the cabled strands during transient conditions. It is the purpose of this thesis to investigate this hypothesis and determine the importance of current distribution to the overall performance of cable-in-conduit conductors under transient conditions.

The remainder of this chapter will be a review of a few basic properties of superconductors along with an introduction to stability analysis. The important aspects of CICC superconductors will also be outlined and then a brief history of

\footnotetext{
* ITER is the International Thermonuclear Experimental Reactor, a joint project of the American, Russian, Japanese and European fusion programs, and TPX is the Tokamak Physics Experiment being planned by the U.S. fusion community.
} 


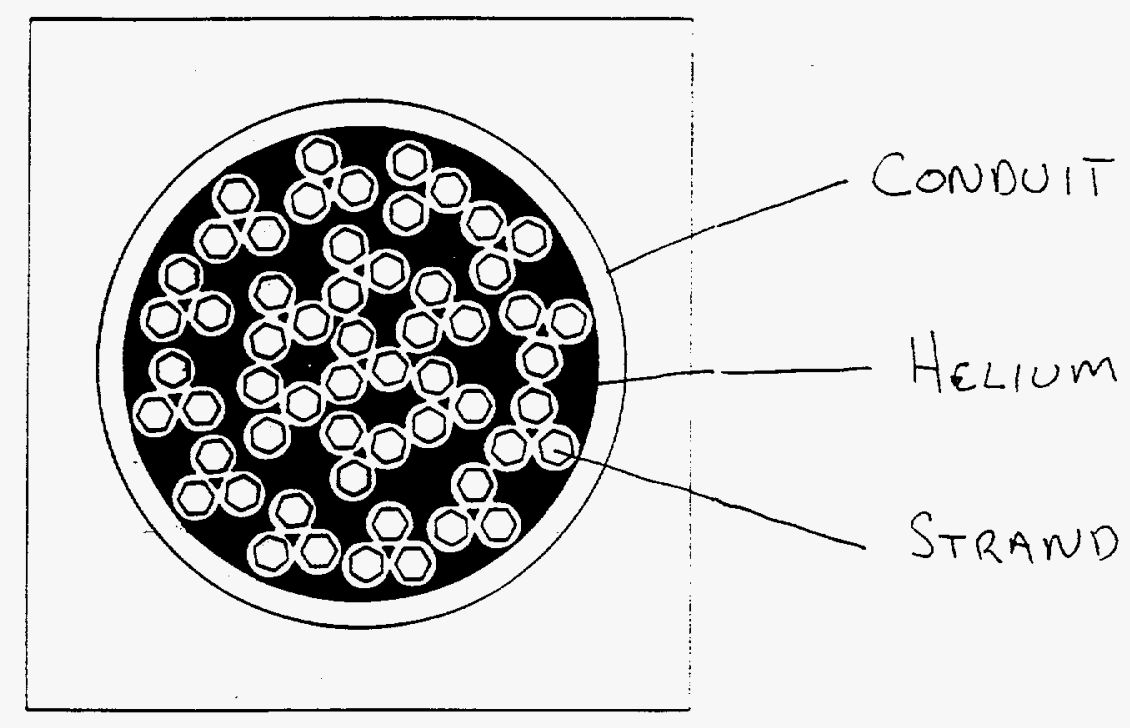

Figure 1.1 An example of a Cable-in-Conduit Conductor (CICC) shown in crosssection. Typically, the strands are twisted into triplets which are further twisted into bundles which are then twisted into sub-cables and so on until the entire cable is formed. Each strand consists of numerous superconducting filaments (not shown in this figure). (Hoenig [1980])

the Ramp Rate Limitation phenomenon will be given, along with some of the possible explanations of the phenomenon.

The second chapter will focus on the patterns of current distribution in CICC magnets using a type of cable in which the strands are multiply-twisted. The chapter begins with a brief discussion of the features of two experimental magnets before developing a discrete numerical model of the simpler of the two. Although the model is specific to one specific cable configuration, a similar model could be applied to other cable geometries. Using the model, patterns of current distribution are studied for a number of scenarios. Each scenario is distinguished by three characteristics: length scale, time scale, and forcing function. Here, forcing function refers to either a linearly-ramping current or a linearly-ramping magnetic field. Different methods of solution are required for each scenario and each will be presented. More detailed results can be found for 
cables considered to be of a periodic nature and infinite in length, but it is shown that current imbalances amongst strands is much greater for finite-length cables terminated in "idealized" joints.

The third chapter will use the results of Chapter Two to form a new stability analysis of the Ramp Rate Limitation. Traditional stability analyses assume a uniform current distribution and focus on the temperature of the cable as the stability criterion. The new analysis will relate the calculated current imbalances to the critical current of a single strand to form a new criterion based on current distribution. Although this analysis makes many simplifications, its predictions reasonably match experimental data.

\subsection{Background}

This section reviews the relevant features of cable-in-conduit conductors (CICC) and introduces the concept of Ramp Rate Limitation. Some basic properties of superconducting materials themselves are first discussed because they are the basis of any stability analysis.

\subsubsection{Basic Properties of Superconducting Materials}

Superconducting materials exhibit their unique properties when operated within certain limits. Traditionally, these limits are defined as the critical temperature, $\mathrm{T}_{\mathrm{C}}$; the critical current density, $\mathrm{J}_{\mathrm{C}}$; and the critical magnetic field, $\mathrm{B}_{\mathrm{C}}$. These three quantities are interdependent and form a three dimensional "critical surface" in a phase space with coordinates of temperature, magnetic field, and current density. The critical surface defines the boundary between the superconducting state (below the surface) and the normal state (above the surface). The critical surface can be considered a material property of the 
superconductor although it is affected by manufacturing techniques. The critical surface for a typical Nb-Ti alloy is shown in Figure 1.2 as an example.

Any superconducting device must be designed so that the superconductor remains "comfortably" beneath its critical surface in phase space. Any disturbance in temperature, field, or current density which moves the operating point above the critical surface will cause the superconductor to go normal. This process is commonly called "Quenching." Thus, the stability of the device against quenching is directly related to how close the nominal operating point is to the critical surface.

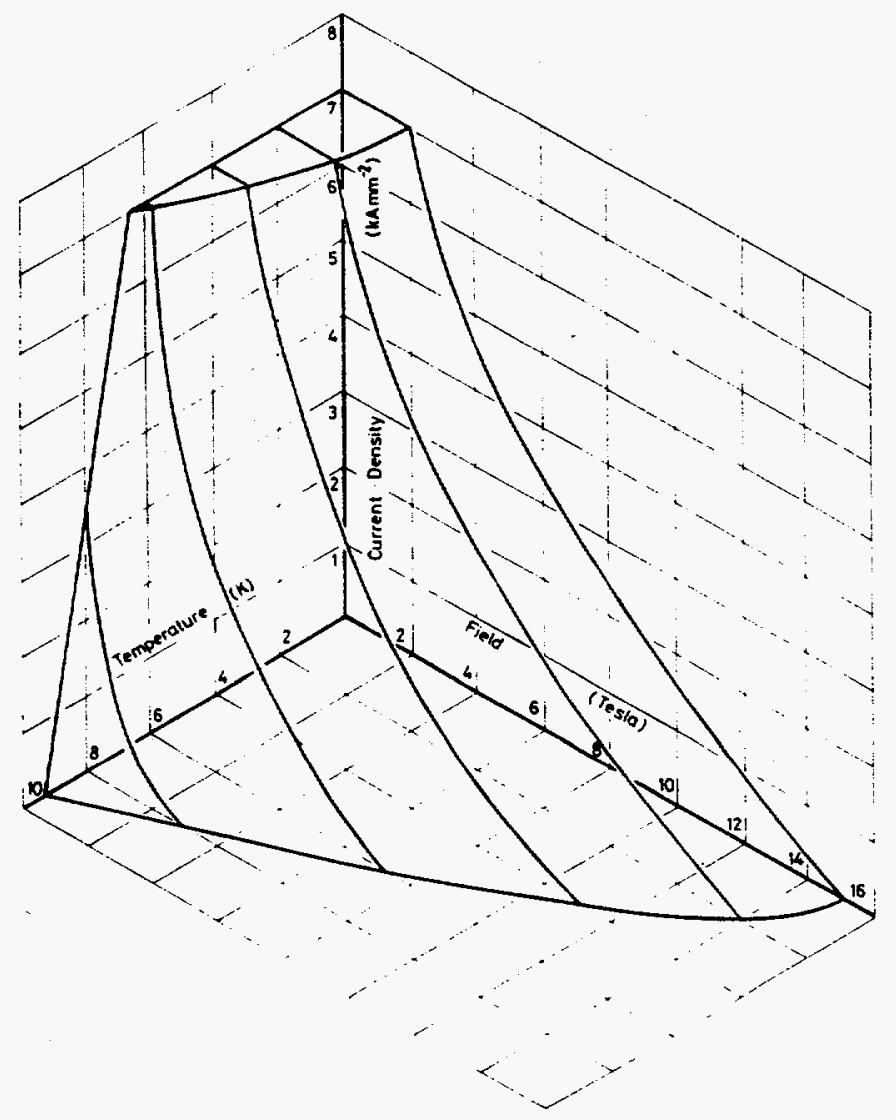

Figure 1.2 The critical surface plot for a commercially available Nb-Ti alloy. At points below the surface, the alloy is superconducting, above the surface, it is normal. Source: reprinted with permission from Wilson, Superconducting Magnets, Copyright (C) 1983 by Oxford University Press. 
The properties of a material in the superconducting state are very different from its properties in the normal state. For stability analysis, the most important difference is the sudden change in the electrical resistivity. Surprisingly, most materials which possess a zero-resistivity superconducting state have relatively high resistivities in the normal state. At cryogenic temperatures, the normal state resistivity of practical superconductors is $10-100 \mu \Omega-\mathrm{cm}$ while, for comparison, the resistivity of copper is less than $0.1 \mu \Omega-\mathrm{cm}$ (Orlando [1991]). For this reason, and since superconductors are usually operated at very high current densities (often greater than $10^{9} \mathrm{~A} / \mathrm{m}^{2}$ (Wilson [1983])), any local transition to the normal state will be associated with tremendous joule heating. Such local joule heating would quickly and irrevocably drive the surrounding superconductor above the critical temperature and into the normal state.

To reduce this "catastrophic" effect, superconducting strands of wire are formed as a composite material: superconductor plus stabilizer. In the event of a local normal spot in the superconductor, the current can flow around the high resistivity region by traveling through the stabilizer (typically made of copper). The joule heating associated with a normal zone is thus greatly reduced. In a good design, a coolant (typically liquid helium) will be able to absorb the heat generated and cool the strand back down to the operating temperature, where it will again be superconducting. This is known as "Quench Recovery."

A stability analysis of a superconducting system determines the size of disturbance which will cause a "Quench" and whether it will lead to a "Quench Recovery" or continue to quench the entire conductor. Traditional stability analyses usually study the stability of a conductor with respect to disturbances in temperature. The current density and magnetic field are assumed to be uniform and constant. Computer Codes such as HESTAB (Bottura, Minervini [1987]) iteratively determine the minimum disturbance energy which would cause the 
wire to joule heat to a point where the temperature remains above the critical temperature despite convective cooling provided by the liquid helium.

The applicability of such a stability analysis to the problem of ramp rate limitation will be discussed at the end of this chapter.

\subsubsection{Cable-in-Conduit Conductors}

Cable-in-Conduit Conductors (CICC) were developed at MIT in the mid1970 's as the initial step in developing large superconducting magnets for fusion reactors and magneto-hydrodynamic (MHD) generators (Hoenig [1980]). In the CICC design, multiple strands of superconducting wire are cabled together and enclosed in a conduit which provides structural support as well as a leak-tight passage for helium coolant. The principal advantage of this geometry is that the surface area to volume ratio is much higher than that of a "monolithic" design. The increased surface contact with the helium coolant provides improved stability with respect to perturbations in temperature. This and other advantages of CICC's for large scale applications are discussed in Hoenig (1980).

The multiple strands of a CICC introduce new concerns for magnet applications. Unless completely insulated, the strands are in electrical contact with their neighbors and there are paths for currents to flow from strand to strand. If the strands are not twisted, induced loop voltages will be proportional to the field rate of change and to the dimensions of the cable (Faraday's Law). Since cables can be very long, significant induced voltages can occur even for small-diameter cables in slowly changing fields. These voltages will drive eddy currents which travel along the length of one superconducting strand and return down the length of a neighboring strand. The only resistance encountered is at the contact points where the current traverses strands. If this resistance is low, notable eddy currents can exist and the resulting joule heating will significantly 
contribute to the AC losses in the cable. This loss must be compensated by additional cooling to maintain the conductor below the critical temperature (Wilson [1983]).

$\mathrm{AC}$ loss is a particular concern for $\mathrm{AC}$ magnets, but is also of interest for DC magnets which must be brought from zero magnetic field to their operating magnetic field in a reasonable amount of time. Fortunately, twisting the strands together effectively reduces the magnetic coupling between strands, putting a handle on AC loss. Twisted strands in a changing external magnetic field experience an electric field which changes direction every half twist pitch length. The maximum induced voltages will thus be proportional to the twist pitch length rather than the length of the cable (Wilson [1983]).

Another way to reduce the coupling between strands is to decrease the conductivity between them. The optimum choice of electrical conductivity between the strands balances the requirement of low AC loss (low conductivity) and adequate stability (high conductivity--to ease current transfer around local normal zones) (Wilson [1983]). For magnets that are designed to be ramped or cycled in time, $\mathrm{AC}$ loss is a primary concern and highly resistive oxide coatings are often used. Completely insulating the strands from each other is an option, but experience has shown that the performance of such magnets can be "surprisingly low" (Turck [1992]).

Although a simple twisting of all the strands, as diagrammed in Figure 1.3, reduces eddy currents due to transverse magnetic fields, it does not help to reduce self-field effects. The self-field is the magnetic field of a wire generated by the transport current flowing through it. The self-field effect in multi-strand cables is similar to the "skin-effect" in homogeneous conductors--a diffusion process in which any change in current distribution begins at the surface and diffuses inward at a rate inversely proportional to resistivity (Haus, Melcher 


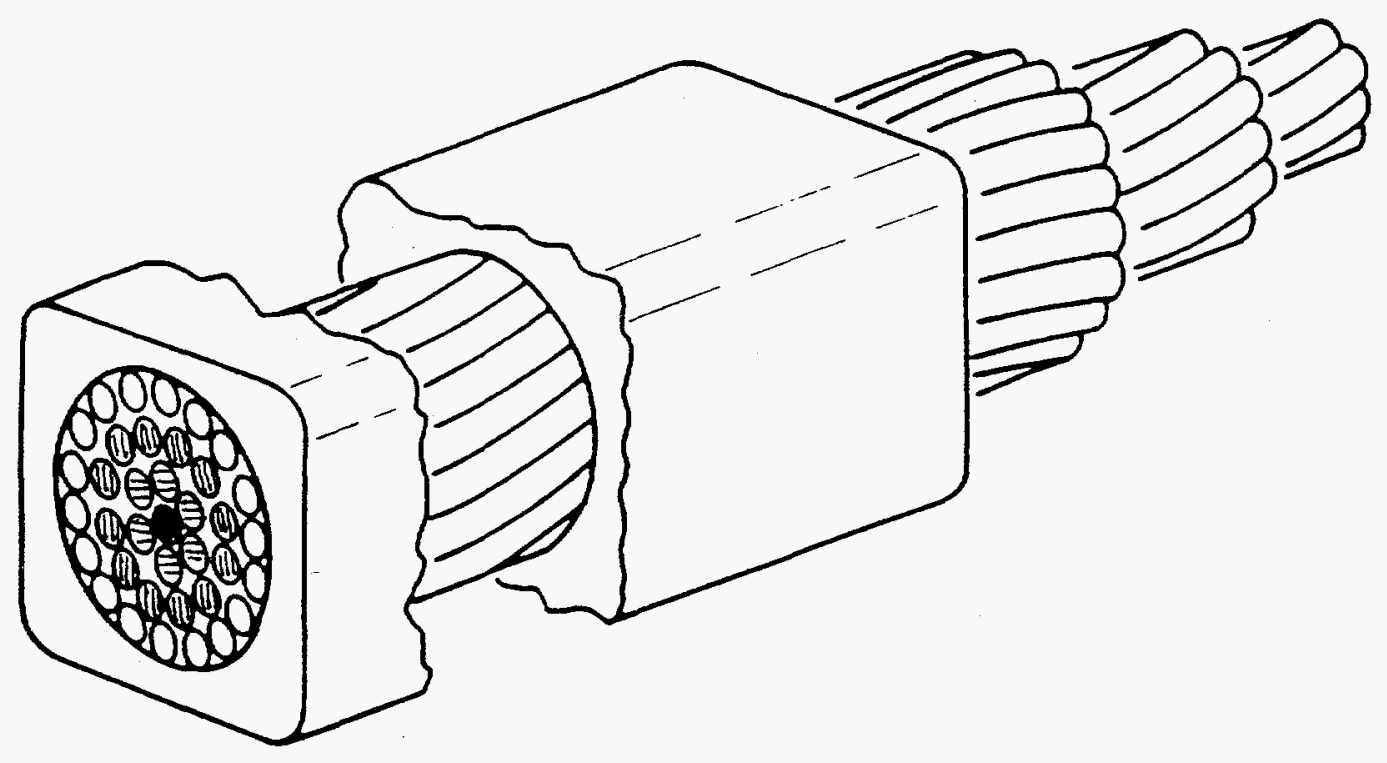

Figure 1.3 A "simply twisted" cable. Strands on the outside remain on the outside over the length of the cable. Strands near the center stay near the center. (Hoenig, et al., [1975])

[1989]). By analogy, any change in the current distribution in a cable will first be felt by strands on the outside before diffusing inward at a rate inversely proportional to the contact resistance. The high contact resistance preferable for reducing $\mathrm{AC}$ losses would therefore unfortunately limit the rate at which current diffuses from outside strands inward in time-varying fields. The resulting current imbalance would reduce the overall performance of the cable.

A "fully transposed" cable, however, is one which eliminates the self-field effect by ensuring that no net self-field flux exists between the strands (Wilson [1983]). This is equivalent to saying that the self-inductance of every strand is the same and that the mutual-inductances between strands exactly balance. This can be achieved by a cabling pattern in which the strands traverse the cable cross-section in such a way that each spends an equal amount of time at each position in the cable space--i.e., the strands spiral radially inward then outward along the length of the cable. 
One such cabling pattern which achieves a fully transposed geometry is a derivative of the Litz wires often used for radio frequency work (Wilson [1983]). It begins by twisting a small number of wires into a "rope" with a tight twist pitch. Then several small ropes are twisted together with a somewhat looser twist pitch into a bundle. The process continues, twisting bundles into larger cables with looser twist pitches. An example of a cable made of five ropes of four strands each is shown in Figure 1.4. The Cable-in-Conduit Conductors discussed later in this thesis are similarly comprised of multiply-twisted strands. It should be mentioned that a cable of this nature is only fully transposed if its final length is an integer multiple of the transposition length, equal to the lowest common multiple of the twist pitch lengths of each stage of the cable. If this criterion is not met, the self-field effects are dependent on the difference between the final length and the closest integer multiple of the transposition length. To minimize this quantity, the twist pitch length of each stage can be made an

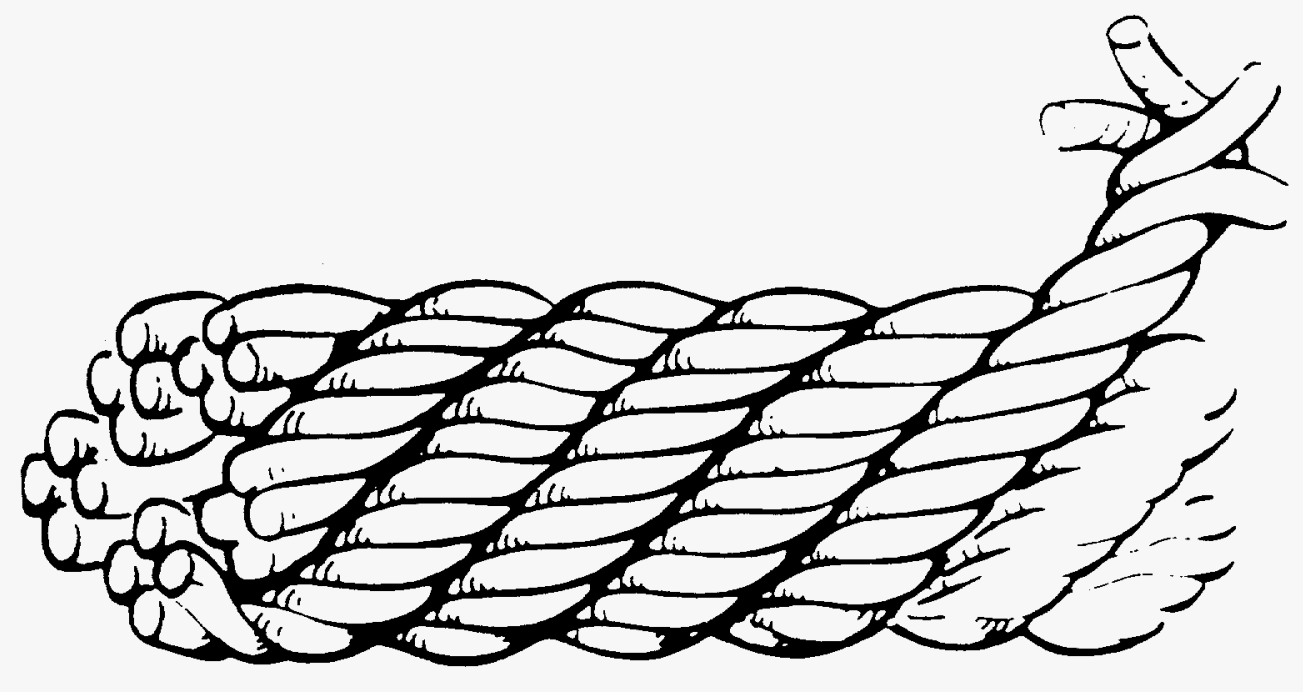

Figure 1.4 A "fully transposed" cable in the form of a multiply twisted "rope." Source: reprinted with permission from Wilson, Superconducting Magnets, Copyright $₫ 1983$ by Oxford University Press. 
integer multiple of the previous stage twist pitch so that the transposition length is as small as possible.

\subsubsection{Ramp Rate Limitation}

To demonstrate the ability of CICC magnets to operate at the conditions required for a fusion device, the United States Demonstration Poloidal Coil (USDPC) was designed and built by MIT and industrial partners. Tested in Japan in late 1990, the magnet performed well in DC tests but exhibited an unexpected ramp rate limitation when ramped at high rate to high currents and fields. Figure 1.5 shows the measured limit for linear current ramping as a plot of maximum attainable current versus the ramp time taken to reach that current. It is evident from the figure that the design current of $\sim 30 \mathrm{kA}$ could only be reached at slow ramp rates (i.e., longer ramp times) despite the fact it was designed to ramp to full current in approximately one second (Painter, et al. [1992]). Although there has been much conjecture about the cause of the limitation (see, for example, Takayasu, et al. (1992), Bruzzone (1992), Bromberg (1993), MIT Plasma Fusion Center (1991)), a consensus of opinion has not been reached.

To further study the problem experimentally, small scale experiments were conducted by the MIT Plasma Fusion Center using the facilities of the Francis Bitter National Magnet Lab. These tests used 27 strand cables made of the same wire of which the 225 strand US-DPC was built. Again, a well-defined ramp rate limit was discovered (Takayasu, et al. [1992]). The results of the simulation of the US-DPC test are shown in Figure 1.6, where solid circles signify points at which the cable quenched and open circles signify currents which were achieved without quench. The Ramp Rate Limitation is again apparent. Although faster ramp rates are known to increase the $\mathrm{AC}$-losses associated with superconductors in changing fields and currents, these losses are 


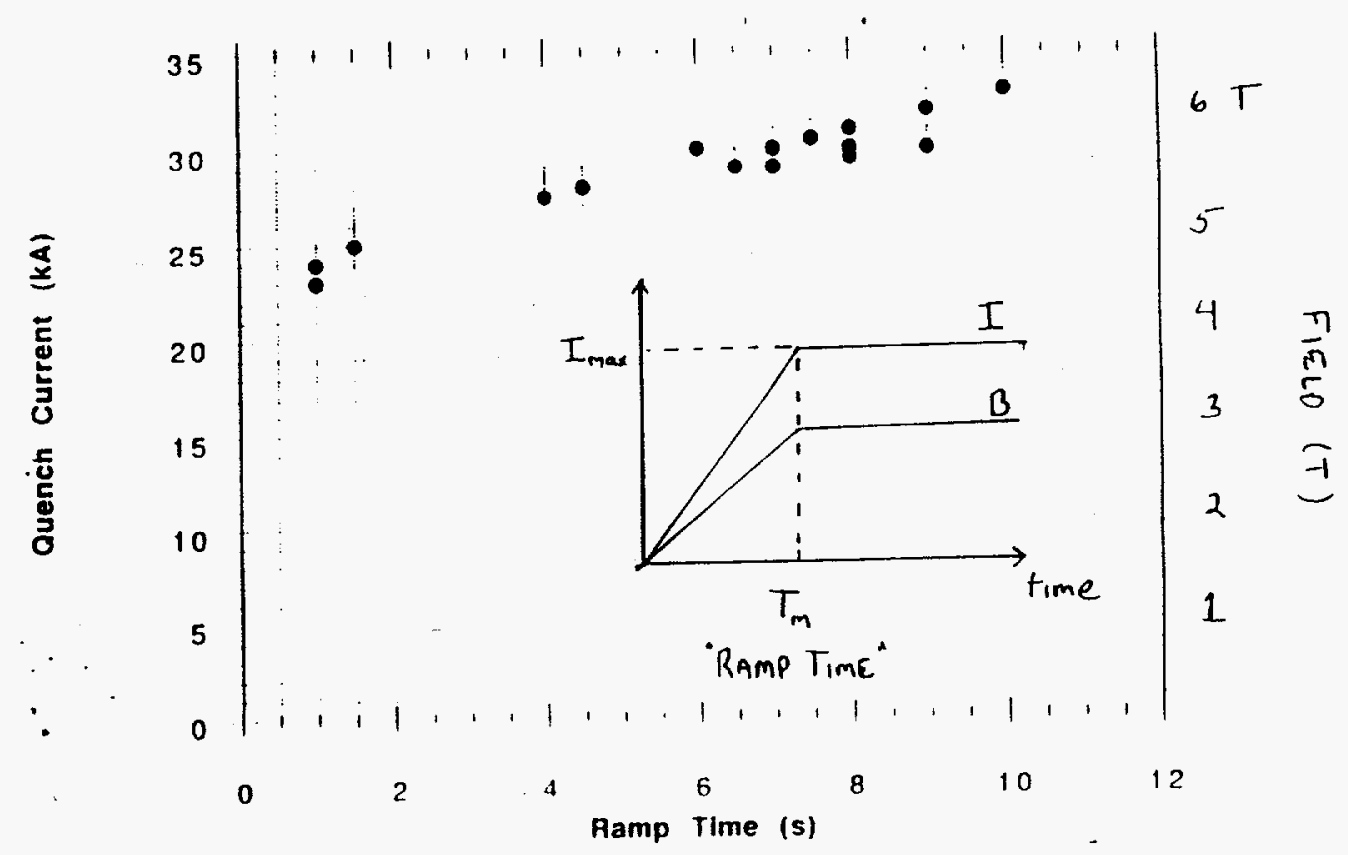

Figure 1.5 Quench current vs. ramp time for the US-DPC experiment. The time developments of the current and magnetic field are shown (inset). The degraded performance at faster ramp times is known as the "Ramp Rate Limitation."

(Painter, et al., [1992])

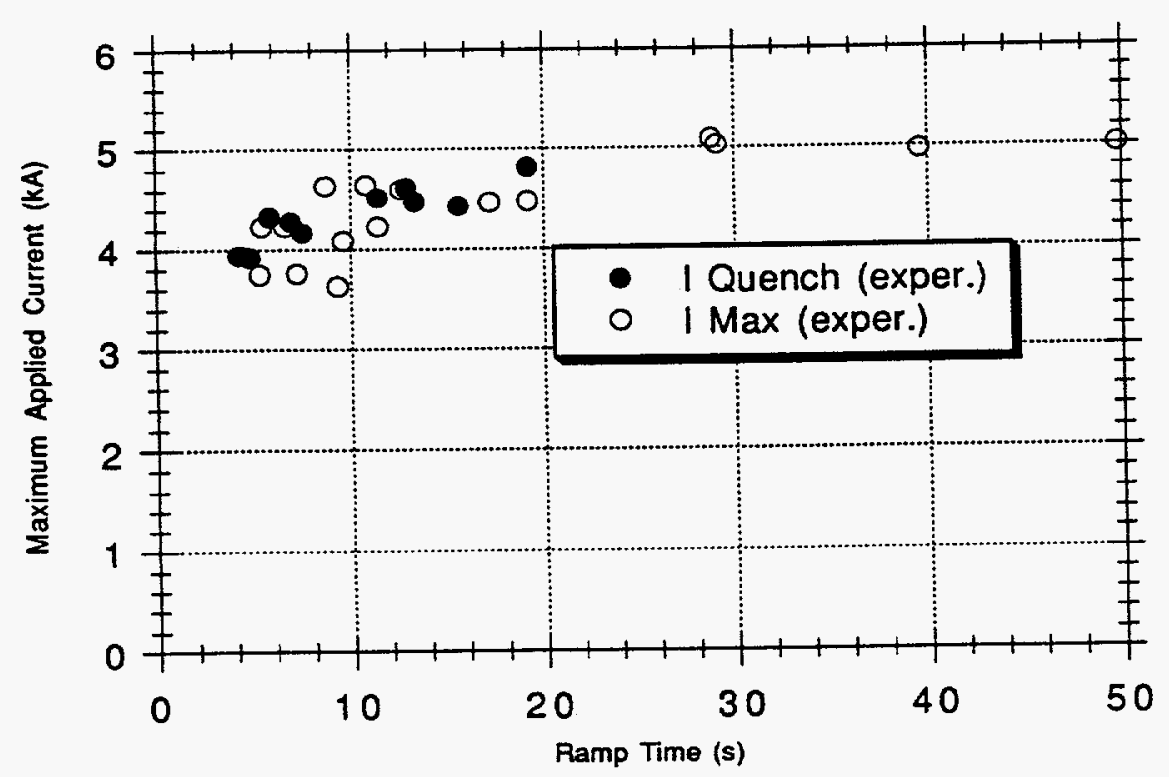

Figure 1.6 Results of the 27 strand US-DPC simulation tests also exhibited a "Ramp Rate Limitation." Solid circles $(\bullet)$ represent points at which the cable quenched during a linear ramp to the quench current in time, tramp. Open circles $\left(^{\circ}\right)$ represent currents which were reached without quench for ramp time, tramp. (Takayasu, et al., [1992]) 
not large enough to explain the degree of degradation encountered at shorter ramp times (Painter, et al. [1992]).

Similar experiments (Vysotsky [1993]) have shown that the Ramp Rate Limitation is due to an instability and not an integrated loss of any kind. This was shown by starting the magnet at an initial bias current and then ramping to the quench current. The quench current was found to be dependent only on the ramp rate, not the initial current. For this reason, it seemed appropriate to study Ramp Rate Limitation using a stability analysis of the CICC.

As mentioned above, traditional stability analyses usually study the stability of a conductor to disturbances in temperature. From these studies, it can be determined if the cooling (heat transfer to helium) is enough to bring the conductor back down to its operating temperature after any foreseeable perturbation in temperature. Such perturbations would be caused by energy deposited in the strands from such causes as AC losses, nuclear heating, or frictional heating associated with wire motion. For this reason the measure of stability of a cable is often given as a "stability margin"--the volumetric energy perturbation which would marginally lead to a non-recovering quench, typically measured in mJ/cc of wire (Bottura, et al. [1991]).

Stability codes which determine the stability margin of CICC conductors are now heavily used design tools for steady-state magnet applications. They are even accurate enough to predict the complicated dual-stability regimes encountered in certain CICC designs (Bottura, Minervini [1991]). In studies of the US-DPC coil and the sub-sized 27 strand cable, however, the stability margin was found to be on the order of $100 \mathrm{~mJ} / \mathrm{cc}$ for operating scenarios at which premature quench was experienced (Steeves, Minervini [1991]). This amount of energy is much larger than any foreseen energy deposition in either system 
(Painter, et al. [1992]). For this reason, the source of the Ramp Rate Limitation is still a mystery.

The standard stability analyses preferred to date may fail to predict a Ramp Rate Limitation because they were not originally designed to model transient current and field conditions. In ramping magnetic and electric fields, the assumptions of uniform field and current distribution inherent in traditional analyses may break down. If significant eddy currents or self-field imbalances exist in the cable, some strands may carry higher than expected currents and thus have a lower than predicted "local" stability margin. In some cases, the cable may only be as stable as the most unstable strand. It is this possibility which will be investigated in this thesis.

A similar effect has already been explored for single-strand superconductors. To correctly predict the performance of single strands in ramping electric and magnetic fields, it is first necessary to use continuum electrodynamics to determine the current and field distribution in the strand. A stability theory which includes these effects and the verifying experiments are presented in Mints, Rakhmanov (1988).

As in the single strand case, to investigate stability of cables in ramping fields it is necessary to determine the current distribution amongst the strands. Chapter Two of this thesis thus studies current distribution in multiply-twisted cables before a "new stability analysis" based on those results is presented in Chapter Three. 



\section{Chapter 2}

\section{Current Distribution in Linearly Ramped Cable-in-Conduit Superconducting Magnets}

\subsection{Overview}

Having explained in Chapter One why knowing the current distribution may be important in determining stability limits, Chapter Two now looks at finding the distributions for the cases of linearly-ramping external magnetic fields and linearly-ramping transport currents. After determining what current imbalances may occur, the stability implications are investigated using simplified stability modeling in Chapter Three.

Many authors have studied current distribution in cables and have determined that the effects can be appreciable (for example, Knoopers, et al. (1985), Amemiya, et al. (1992) and Torii, et al. (1993)). Unfortunately, all previous reports have used simplifying assumptions which limit the applicability of their results for the problem of CICC's. Specifically, the effects of the complicated, twisted nature of the cabled strands and the effects of transverse conductance between the strands have not yet been treated appropriately. This chapter will include these effects in numerical models which give a more accurate description of current distribution in multiply-twisted cable-in-conduit conductors.

\subsubsection{The Experimental Magnets}

The US-DPC magnet is in many ways typical of CICC magnets. The magnet is formed as a pancake wound solenoid in which the magnetic field produced in the bore is linearly related to the current. Correspondingly, the rate of change of the field is proportional to the rate of change of the current. The 
magnetic field experienced by the conductor, however, is not uniform over the cable length and this field distribution would have to be included in any study of induced currents. The details of the magnet design and field distribution can be found in reference (Painter, et al. [1992]).

The operating conditions of the US-DPC experiment were simulated in the small-scale 27 strand experiment by simultaneously ramping a transport current through the cable and a background field provided by a $12 \mathrm{~T}$ Bitter magnet (Takayasu, et al. [1992]). Unlike the field produced by the US-DPC, the background field at the test sample is uniform over the length of the conductor. The relation between the background field B and transport current I was fixed in such a way that the current of each strand of the 27 strand cable was equal to that of the US-DPC coil (225 strands) at a given magnetic field. The experimental setup is shown schematically in Figure 2.1.

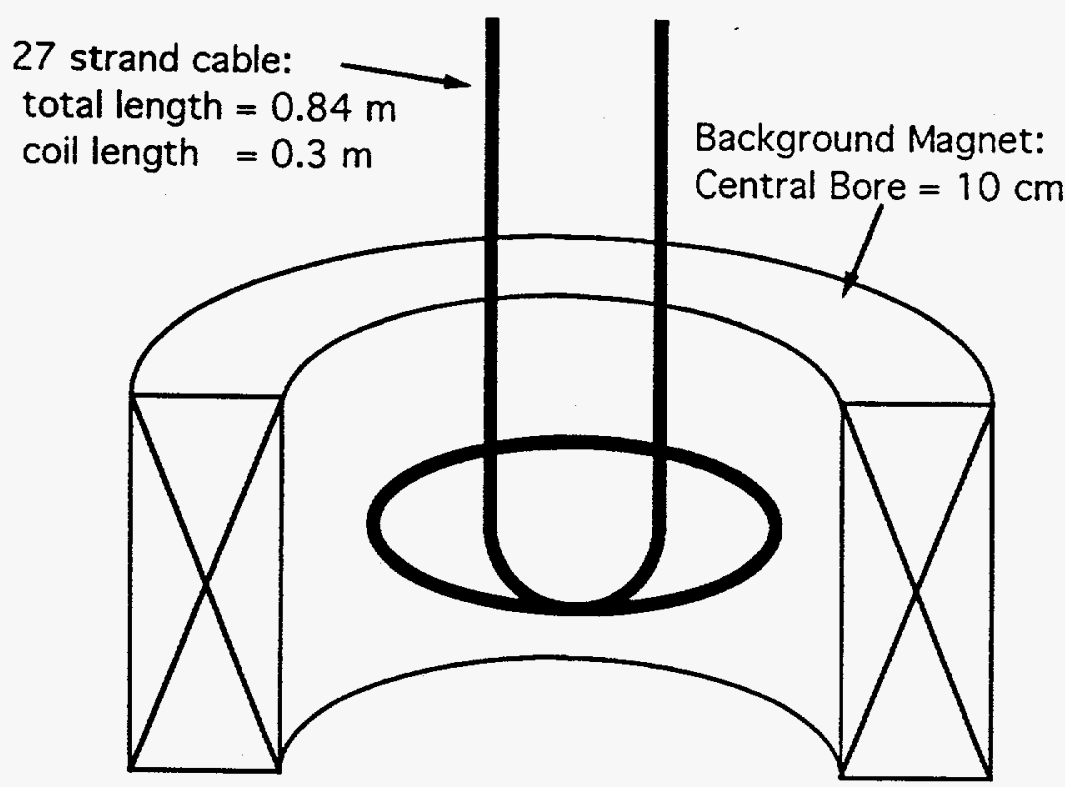

Figure 2.1 Schematic of the 27 strand US-DPC simulation experiment. A single loop coil formed from the CICC was placed in the bore of a $12 \mathrm{~T}$ Bitter magnet. The ratio of background field to transport current was kept constant as they were both linearly ramped in time. 


\subsubsection{Causes of Uneven Current Distribution}

A non-uniform current distribution amongst the cabled strands of a CICC conductor can be caused by several factors. In general, unless each strand provides an identical current path in terms of its resistive and inductive properties, imbalances will exist.

\section{Uneven Joint Resistances}

The superconducting cable has to be connected to the power supply bus bar in a specially designed joint. These joints are designed to minimize total crossover resistance and thus small differences in the resistance of individual strands to the bus bar can be relatively large. Although this is a valid concern, a poorly manufactured joint would affect the DC performance of a magnet as well as the ramping performance. Since the DC performance of the magnets studied in ramp rate experiments showed no degradation, (Painter, et al. [1992]) the resistive imbalances are not considered to be a contributing factor to Ramp Rate Limitation and are not explicitly discussed in this thesis.*

\section{Self-Field Effects}

As mentioned above, the "self-field" effect is similar to the "skin effect." CICC conductors use almost fully transposed cabling patterns which can potentially eliminate self-field effects but, unless the cable is perfectly periodic and its length is exactly equal to an integer multiple of the transposition length, self-field effects will not be completely canceled. Also, the presence of transconductance along the length of the cable allows the current to "tend outward" locally even if each strand uniformly weaves in out of the center of the cable. These local effects are transient in nature, though, and the overall current

\footnotetext{
"Recently published results show a frequency dependency for the "current sharing length"--the length scale over which current imbalances due to any uneven joint resistances re-equilibrate (Kawabata, et. al. [1994]).
} 
distribution will be related to whole length effects at sufficiently long times for linearly-ramping currents. This process is discussed further below.

\section{External-Field Effects}

In a magnet comprised of CICC, the external-field is defined as the field due to currents in strands other than those being studied. In the case of the small-scale experiment, this is the background magnetic field. As mentioned in Chapter One, even though the strands are twisted, flux changes through the tiny linkages (minimized by tight twist pitches) cause current loops to flow along short lengths of strands and through the transverse conductive path where the strands come into contact. Additionally, if the cable length is not a multiple of the final twist pitch length (or if the twist pitches themselves vary over the cable length from their nominal values), flux linkages will not cancel and a changing magnetic field will drive net circulating currents along the length of the strands through the low resistance joints. This net eddy current can be much larger than the local eddy currents encountered in a periodic cable.

\subsection{Approach to Studying Current Distribution}

The 27 strand cable used in the small-scale US-DPC simulation experiments will be used throughout this chapter as the basis for current distribution modeling because of its manageable size and the available experimental database from which to draw comparisons. The 27 strand sample

is typical of other cables, however, and thus the techniques developed can be applied to other cable geometries. 


\subsubsection{Properties of the 27 Strand Experimental Cable}

The cable consists of 27 strands of a Nb3Sn superconducting composite with a strand diameter of $0.78 \mathrm{~mm}$. The strands are plated with chrome-oxide to decrease the trans-conductance and then they are multiply-twisted into a cable of 3 bundles of 3 sub-bundles of 3 strands each. Details of the twisting will be discussed below. The cable is contained in an Incoloy 908 conduit with a $5.5 \mathrm{~mm}$ inner diameter and a $6.7 \mathrm{~mm}$ outer diameter. The cable is $0.84 \mathrm{~m}$ long and was formed into a single-turn coil of $95 \mathrm{~mm}$ diameter; thus $30 \mathrm{~cm}$ of the cable experiences a transverse external-field (Takayasu, et al. [1992]). The geometry is shown in Figure 2.1.

A measure of the transverse conductance between the cabled strands was obtained by Takayasu (1992). The tests were performed on a cable constructed similar to the one above except one of the 27 strands was separated at the terminations and connected to a separate power supply. Using four-point voltage taps (Wilson [1983]), the net conductance between the single strand and the 26 others could be found as a function of background field and transport current. The results were given as a contact conductance per unit length, G, defined as:

$$
G=\frac{1}{R} \quad(\mathrm{mho} / \mathrm{m})
$$

where $R=$ the measured resistance for a $1 \mathrm{~m}$ cable. The measured transconductance was found to be fit well by the expression (Takayasu [1992]):

$$
G=214.7 B * I+2099.0 \quad(\mathrm{mho} / \mathrm{m}),
$$

where $B$ is the background field in Tesla and I is the transport current in kA. The dependence on the product $B^{*} I$ represents the increased conductance due to contractile Lorentz' forces in the cable.

It should be mentioned that other experimenters have attempted to measure contact resistance between chrome-plated strands similar to the ones 
used here but have obtained results which are greater than the correlation in Eq. (2.2) by up to a factor of 1,000 (Egorov, et al. [1992], Turck [1992]). These other experiments, however, used only 2 or three strands instead of a full cable and thus the nature of the contacts between strands may have been quite different. While this discrepancy has not yet been entirely explained, the measurements made on the 27 strand sample will be used exclusively since it is to that cable to which we will primarily compare calculated results.

\subsubsection{Model Assumptions}

The presence of transverse conductance makes the problem of current distribution in multi-strand cables difficult to study analytically and thus numerical techniques will be used instead. The numerical model will make several assumptions:

1. The cable will be assumed to be straight with its longitudinal axis pointing in the $\mathrm{z}$-direction. This assumption will be generally valid for single coil magnets with a coil radius $>>$ cable radius.

2. The external-field will be assumed to be uniform over the length of the cable.

3. Unless otherwise noted, the twist pitches of each cable stage exactly equal their nominal value.

4. The "randomizing" effects of cable compaction on geometry will be neglected, although we do use special Maps which give the "most compact" cable geometries consistent with the nominal twist pitches of the cable.

While assumptions 1 and 2 limit the application of the model to single loop coils in uniform magnetic fields, (as is the case being studied here, with the coil radius of $4.8 \mathrm{~cm}$ much greater than the cable radius of $0.28 \mathrm{~cm}$ ), these assumptions could be relaxed at the price of increasing the detail of the geometric modeling. 


\subsubsection{Discretized Cable Geometry}

Having made the above assumptions, it is possible to generate a geometric model of the cable being studied. As described in section 2.2.1, a typical cable is composed of successive twisted stages, with the twist pitch of each stage an integer multiple of the previous one. In the 27 strand sample, (as in the full USDPC cable) each twist pitch length is double the previous one. We will use the terms triplet, bundle, and cable to refer to the first, second, and last stages of the $3 \times 3 \times 3$ strand cable. The nominal twist pitches of the triplets, bundles, and cable are $51 \mathrm{~mm}, 102 \mathrm{~mm}$, and $203 \mathrm{~mm}$, respectively (Painter, et al. [1992]).

When current distribution within a strand needs to be calculated, a "continuum electrodynamical model" is usually used (Carr [1983]). For a cable however, the distinct strands and the intermittent contacts make the problem a discrete one by nature. Kirchoff's laws (integral forms of Maxwell's Equations) are good for describing discrete systems and will be the tools used here. It is therefore necessary to discretize the cable geometry.

An appropriate geometric model will approximate the paths of the strands to determine: the contact points between the strands, the "local" and "total" external flux linkages, and the mutual-inductances. Although we cannot exactly match the true geometry, such a model will still allow for a meaningful analysis of the cable. The hardest aspect of the cable to model is the nature of the intermittent contacts between the strands. The following model was chosen over simpler models because it was thought to more realistically approximate the inter-strand contacts.

After "trial and error," the final twist pitch length of the cable (equal to the transposition length for the 27 strand cable) was axially discretized into 48 longitudinal segments. The choice of 48 segments was made because it allowed for a representation of the cable which preserves the correct twist pitch rotations 
between segments. The length of each segment is $4.2 \mathrm{~mm}$, the transposition length $(203 \mathrm{~mm})$ divided by 48 .

The configuration of strands at the midpoint of each of the 48 segments is determined by the various twist pitch lengths in the cable. The full cable will complete one poloidal rotation $\left(360^{\circ}\right)$ over one final twist pitch length, equivalent to 48 segments. Thus, the cable has a $7.5^{\circ}$ poloidal twist per segment. The twist pitch length of the second-stage bundle is half that of the cable and, thus, the bundle would nominally twist $15^{\circ}$ per segment. However, the bundle is twisted even further when it is incorporated into the cable, so the two twists must be added to give an actual bundle twist of $22.5^{\circ}$ per segment. Similarly, the nominal triplet twist would be $30^{\circ}$ per segment, but it is then twisted into a bundle and then finally into a cable so we must add the three nominal twist pitches to give an actual triplet twist of $52.5^{\circ}$ per segment.

The Maps:

Figure 2.2 shows a set of strand configurations at the midpoint of each of the first eight segments of the cable model. These maps, labeled Map A through Map $\mathrm{H}$, represent the most compact configurations possible which maintain the prescribed twist pitches for the 27 strand cable with. Moving down the length of the cable, the strand in the position labeled " 1 " in Map A moves to position " 1 " in Map B, and so on until Map H (the eighth segment). The other strands move similarly.

Figure 2.3 shows how the nominal twist pitches are included in the model geometry. The twisting of a stage is defined by the angular rotation of a triangle connecting the centers of the sub-elements of that stage. For example, strands 1,2 , and 3 comprising the first triplet form a triangle which has a $52.5^{\circ}$ twist per segment, as prescribed above. Similarly, for the bundle composed of strands 1-9, the centers of the three triplets-- $(1,2,3),(4,5,6)$, and $(7,8,9)$--form a triangle which 

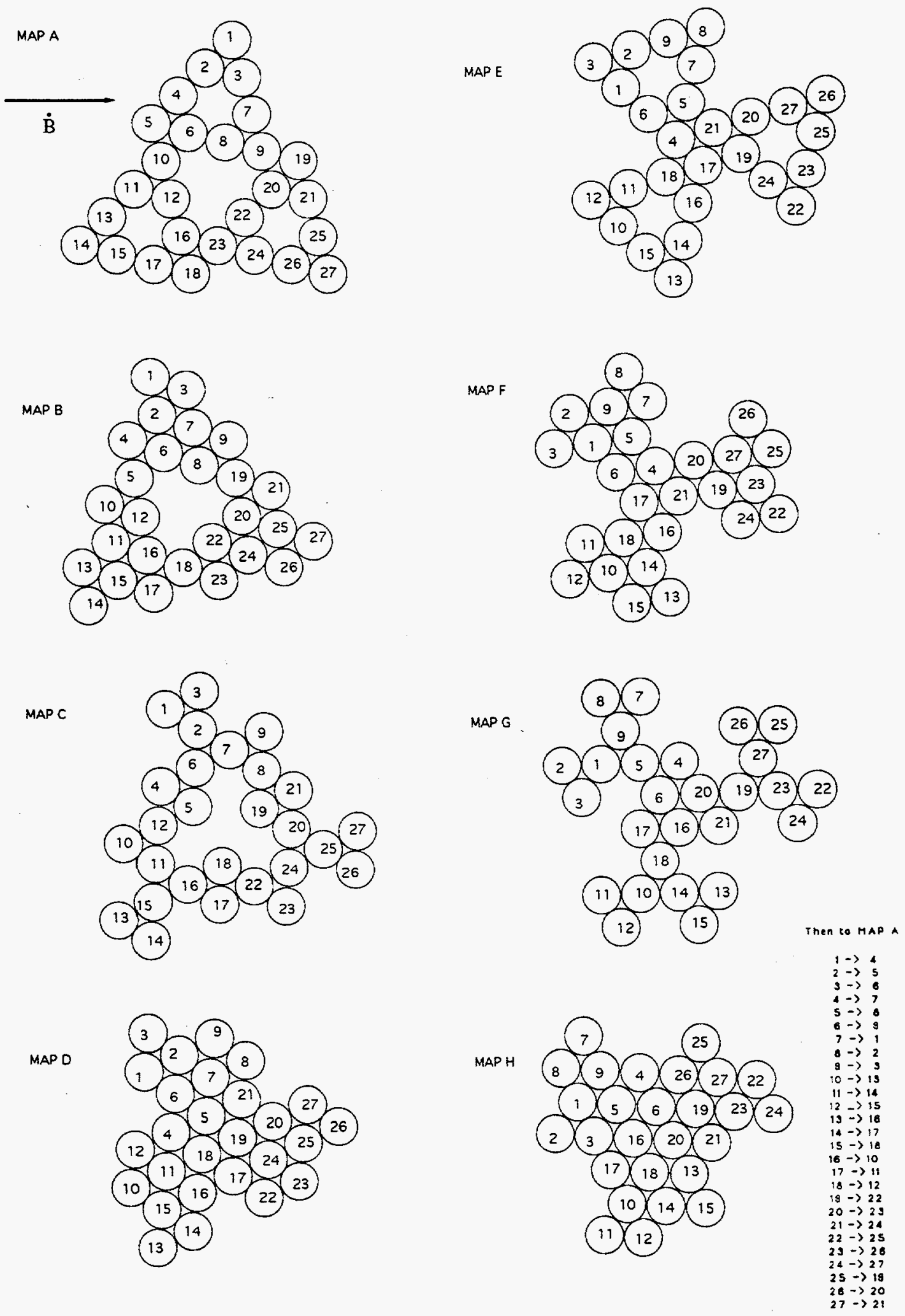

Figure 2.2 The first 8 strand configurations of the 48 segment period of the 27 strand cable model. See text (Section 2.2.3) for explanation. 


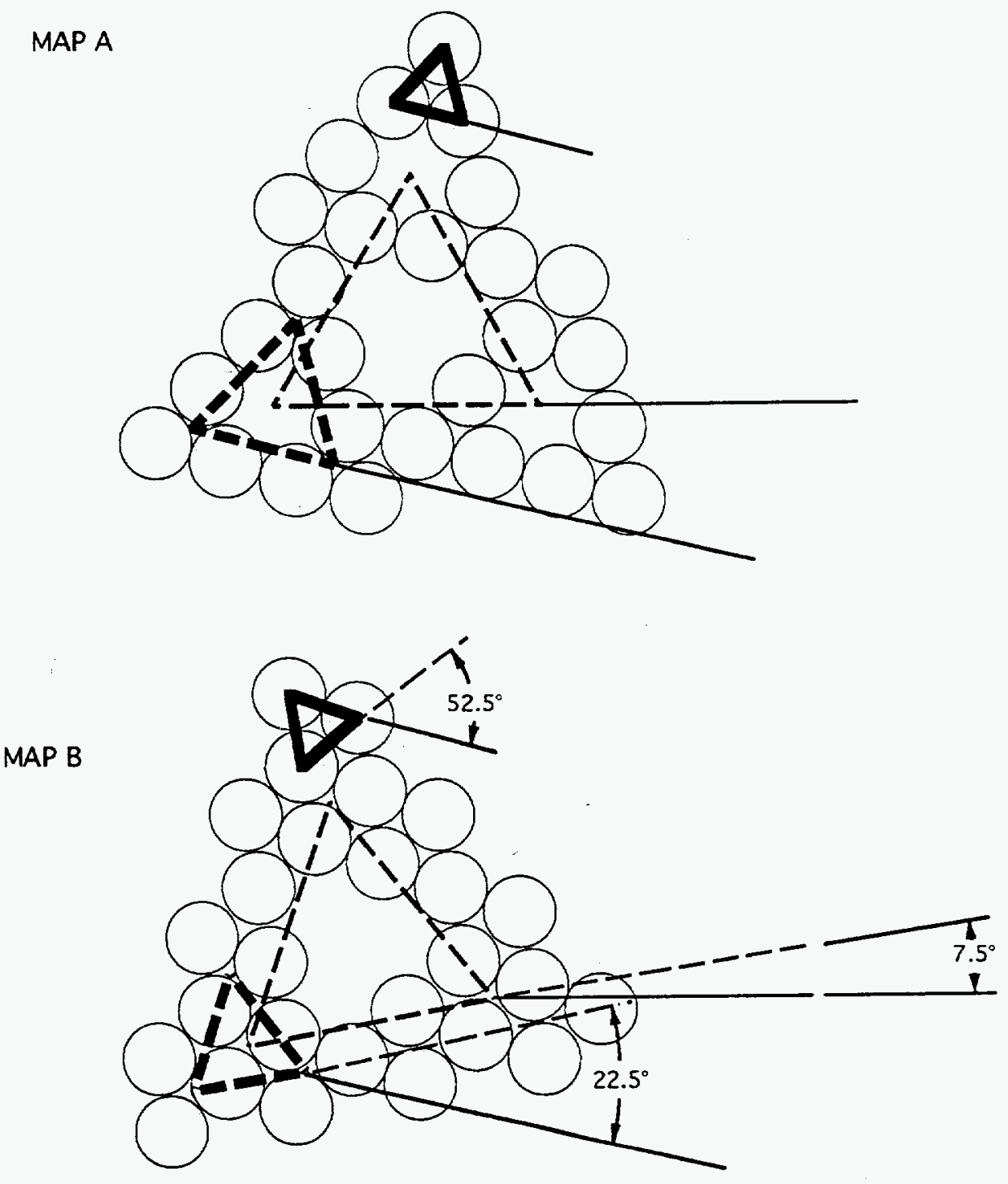

Figure 2.3 Between each segment of the cable, the strands rotate according to their prescribed twist pitches. Each triplet (heavy, solid triangle) rotates $52.5^{\circ}$. Each bundle (heavy, dotted triangles) rotates $22.5^{\circ}$, and the cable (lighter, dashed triangle) rotates $7.5^{\circ}$. See text (Section 2.2.3) for explanation. 
makes a $22.5^{\circ}$ twist per segment. Last, the triangle defined by the centers of the three bundles make a rotation of $7.5^{\circ}$ per segment. Thus, the configurations presented in the Maps preserve the correct multiple twisting nature of the 27 strand cable.

All 48 segments which make up one period of the cable can be represented at their midpoint by Maps A-H from Figure 2.2. The configurations of the cable in the next eight segments of the cable are equivalent to the first eight with a $60^{\circ}$ counter-clockwise rotation added to the orientation of the Maps A-H. Thus, from a segment whose configuration is indicated by $\mathrm{Map} \mathrm{H}$, the axial midpoint of the next cable segment is again in the configuration of Map A, but with a $60^{\circ}$ counter-clockwise rotation. Furthermore, the strand labeled " 1 " in the eighth segment (Map H) now moves to the position labeled " 4 " in the ninth segment (Map A, with $60^{\circ}$ counter-clockwise twist). Similar "translations" for each of the strands continuing from Map H to Map A are given in the figure and in Table 1. Continuing from segment 9 (Map A), the strand in this example proceeds to the position labeled " 4 " in each of the subsequent maps up through segment 16 , (Map H), when it must again be "translated" before proceeding again to Map A (appropriately twisted another $60^{\circ}$ in the counter-clockwise direction). The path of any strand can thus be followed from segment to segment for any length of cable

Note that after traversing 24 segments, a strand will end up in the same map, in the same position from which it started only the Map will have rotated $180^{\circ}$ from its original orientation. This "odd-symmetry" property will be used in solving for the current distribution in the cable in some cases. After 48 segments, the cable returns to the same orientation from which it begins, completing one period. 


\begin{tabular}{||c|c|c|}
\hline$A$ & $B$ & $C$ \\
\hline 1 & 4 & 7 \\
\hline 2 & 5 & 8 \\
\hline 3 & 6 & 9 \\
\hline 4 & 7 & 1 \\
\hline 5 & 8 & 2 \\
\hline 6 & 9 & 3 \\
\hline 7 & 1 & 4 \\
\hline 8 & 2 & 5 \\
\hline 9 & 3 & 6 \\
\hline
\end{tabular}

\begin{tabular}{||c|c|c|}
\hline$A$ & $B$ & $C$ \\
\hline 10 & 13 & 16 \\
\hline 11 & 14 & 17 \\
\hline 12 & 15 & 18 \\
\hline 13 & 16 & 10 \\
\hline 14 & 17 & 11 \\
\hline 15 & 18 & 12 \\
\hline 16 & 10 & 13 \\
\hline 17 & 11 & 14 \\
\hline 18 & 12 & 15 \\
\hline
\end{tabular}

\begin{tabular}{|c|c|c|}
\hline$A$ & $B$ & $C$ \\
\hline 19 & 22 & 25 \\
\hline 20 & 23 & 26 \\
\hline 21 & 24 & 27 \\
\hline 22 & 25 & 19 \\
\hline 23 & 26 & 20 \\
\hline 24 & 27 & 21 \\
\hline 25 & 19 & 22 \\
\hline 26 & 20 & 23 \\
\hline 27 & 21 & 24 \\
\hline
\end{tabular}

Table 1. A strand which is in Map H (Figure 2.2) in the position shown in Column A next proceeds to Map A at the position designated in Column B. The next time it reaches Map $\mathrm{H}$, it continues on to Map A in the position shown in Column C. Continuing onward, the next time the strand passes Map H, it returns to Map A in its original position, again shown in Column $\mathrm{A}$.

See text for further details.

\section{A Comparison between "Pure Twisting" and "Discretized" Models:}

The above model is somewhat complicated but gives a better representation of the contacts between strands than would a simpler model based purely on the twist pitches. Since the geometry of the simpler model is easier to visualize, however, it is presented as a clarifying comparison to the more intricate model used here.

The more basic model starts with the strand configuration shown in Figure 2.4. Proceeding from this initial set-up down the length of the cable, the triplets, the bundles, and the cable all rotate continuously about their respective centers according to their individual twist pitches. In cylindrical coordinates, the position of each strand in the cross-section can be considered a sum of three vectors:

$$
\left(r(z), \theta\left(z_{2}\right)\right)=\left(r_{1}, \theta_{1}(z)\right)+\left(r_{2}, \theta_{2}(z)\right)+\left(r_{3}, \theta_{3}(z)\right)
$$

where the position $\left.\left(r_{2}\right), \theta(z)\right)$ is a function of the axial position and is referenced to an origin at the center of the cable. The vectors $\left(r_{s}, \theta_{s}\left(z_{2}\right)\right), s=1,2$ or 3 , are referenced to the origins shown in Figure 2.4. While each $r_{s}$ is constant, the angles change continuously according to the equation: 


$$
\theta_{s}(z)=\theta_{s}(0)+z \frac{d \theta_{s}}{d z}
$$

where, for each stage $s, \frac{d \theta_{s}}{d z}$ is the rate of twist pitch and $\theta_{s}(0)$ is the angular coordinate at $z=0$.

In Figure 2.5, the resulting path for the strand labeled " 1 " in Figure 2.4 is compared to the path for the strand labeled " 1 " in Figure 2.2. The paths are shown in the plane perpendicular to the cable axis for one period of cable length

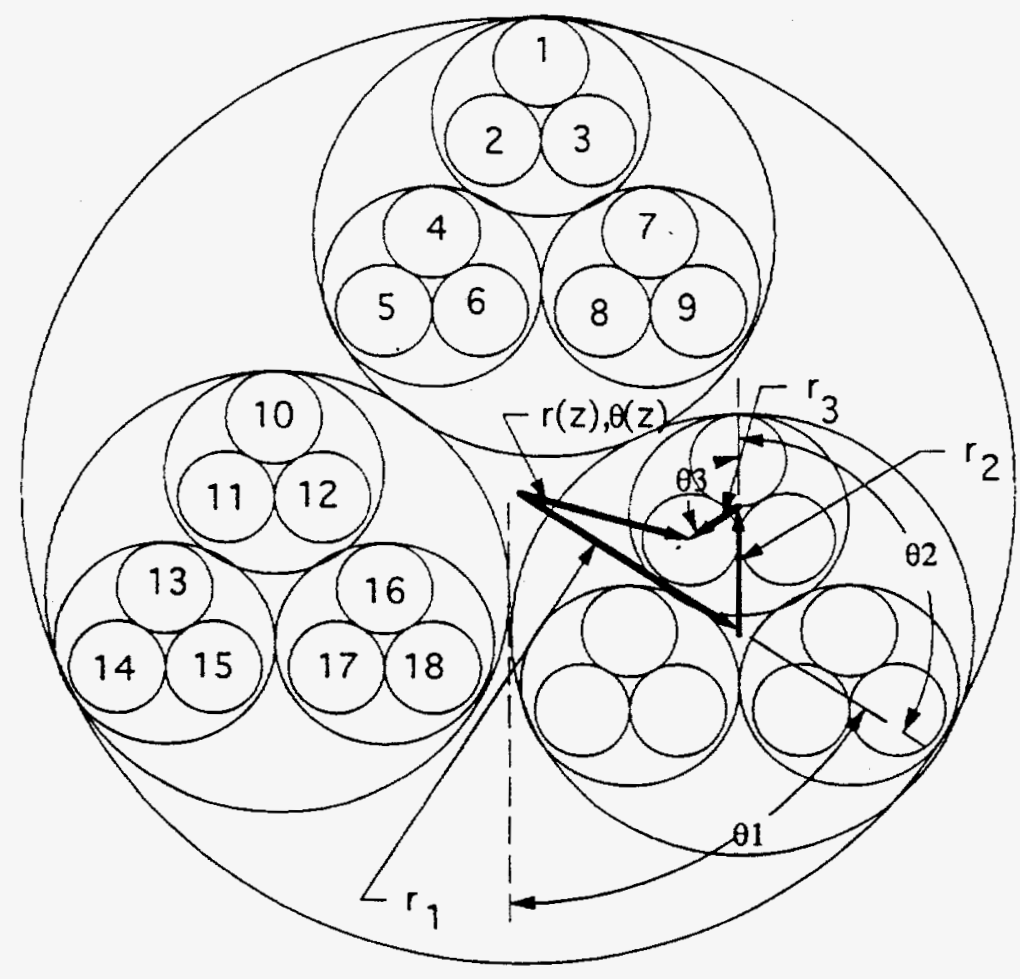

Figure 2.4 The geometry of a simplified representation of the 27 strand cable is shown. The position of each strand in polar coordinates at each axial position $z$ is $(\mathrm{r}(\mathrm{z}), \theta(\mathrm{z}))$ and is a summation of the three rotating vectors which define the individual twist pitches of each stage of the cable, as shown. 

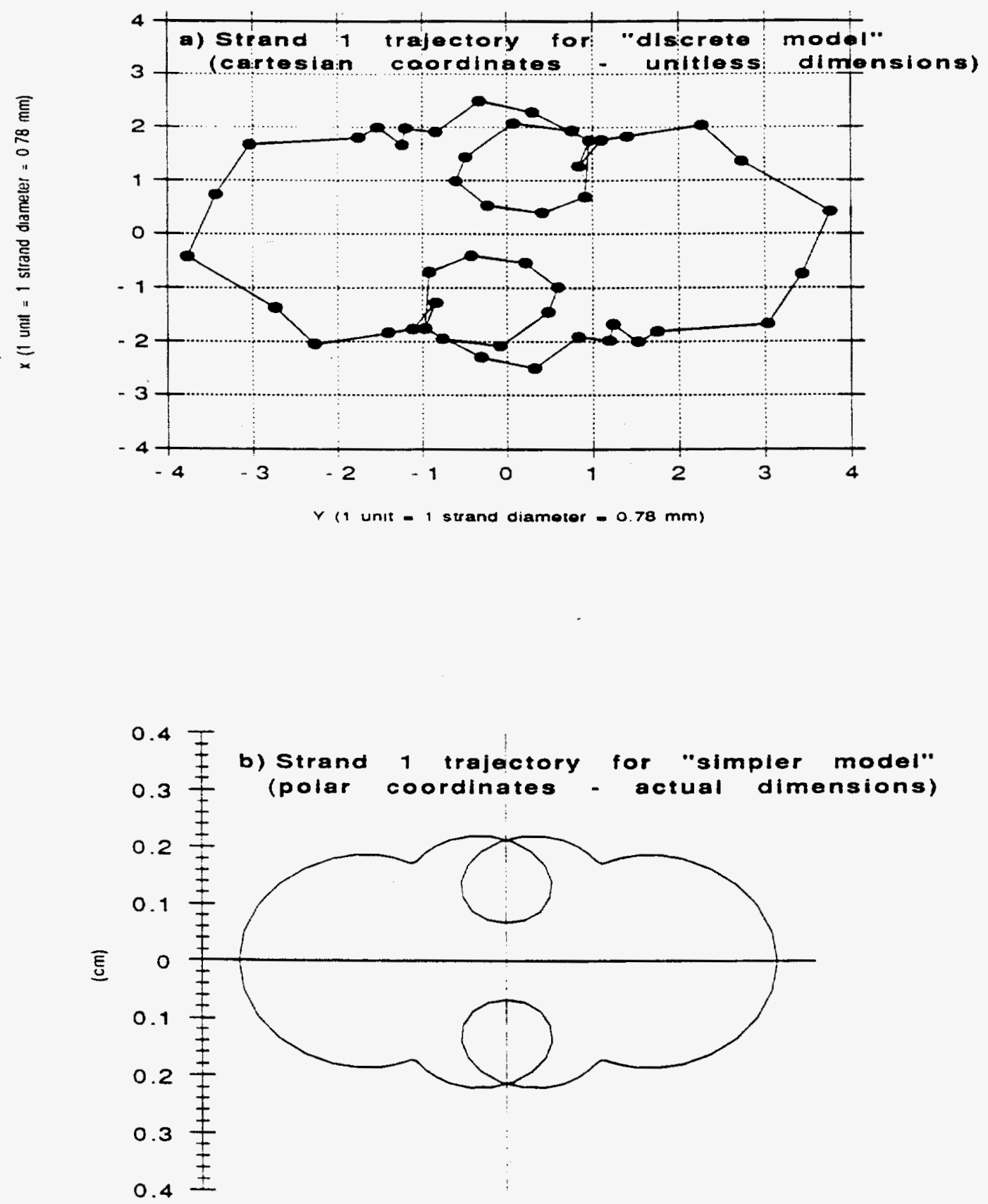

Figure 2.5 A comparison of the trajectory of strand 1 for two different geometric models of a 27 strand CICC: a) the discrete model used in calculations, b) a simpler, continuous model. 
and the similarities are apparent. Although neither of these paths is the true path of a strand in a real-world cable, this comparison shows that the geometric model developed above preserves the intricate geometry of a multiply-twisted cable while allowing for more realistic modeling of inter-strand contacts.

\subsubsection{Circuit Description from Maps}

With the geometry now discretized, the CICC can be described as a "Mesh Current Network." The midpoint of each strand (labeled " 1 " through " 27 " in Maps A-H) is considered a "node" and for a cable 48 segments long (one period), there are $27^{*} 48=1,296$ nodes. Two nodes within the same Map are connected through a contact resistance if they are shown to touch in that Map. Also, two nodes with the same label (" 1 " - " 27 ") in adjacent Maps are connected through an inductive element. (Between Map H and Map A we have to consider the "label translation" required, as shown in Table 1) Each loop which would be formed between contiguous contacts between the same two strands is called a "mesh." Thus, a complicated resistive/inductive network of meshes is formed. A simplified schematic for a partial three strand mesh network is shown in Figure 2.6 to assist in the following description of the different circuit elements.

Parallel and Transverse Currents:

To keep track of all of the currents in the mesh network, it is necessary to number them as well as numbering the nodes. Each node is distinguished by subscripts defining its location: node $i, n$ resides at the $n$-th segment of strand $i$. The currents which travel along the strands are similarly defined by their location: $I_{i, n}$ is the current from node $i, n$ to node $i, n+1$. The contact currents flowing transversely between strands are defined using three subscripts and, to easily

distinguish them from the parallel currents, a lower-case character is used: $i_{\mathrm{i}, \mathrm{j}, \mathrm{n}}$ is 


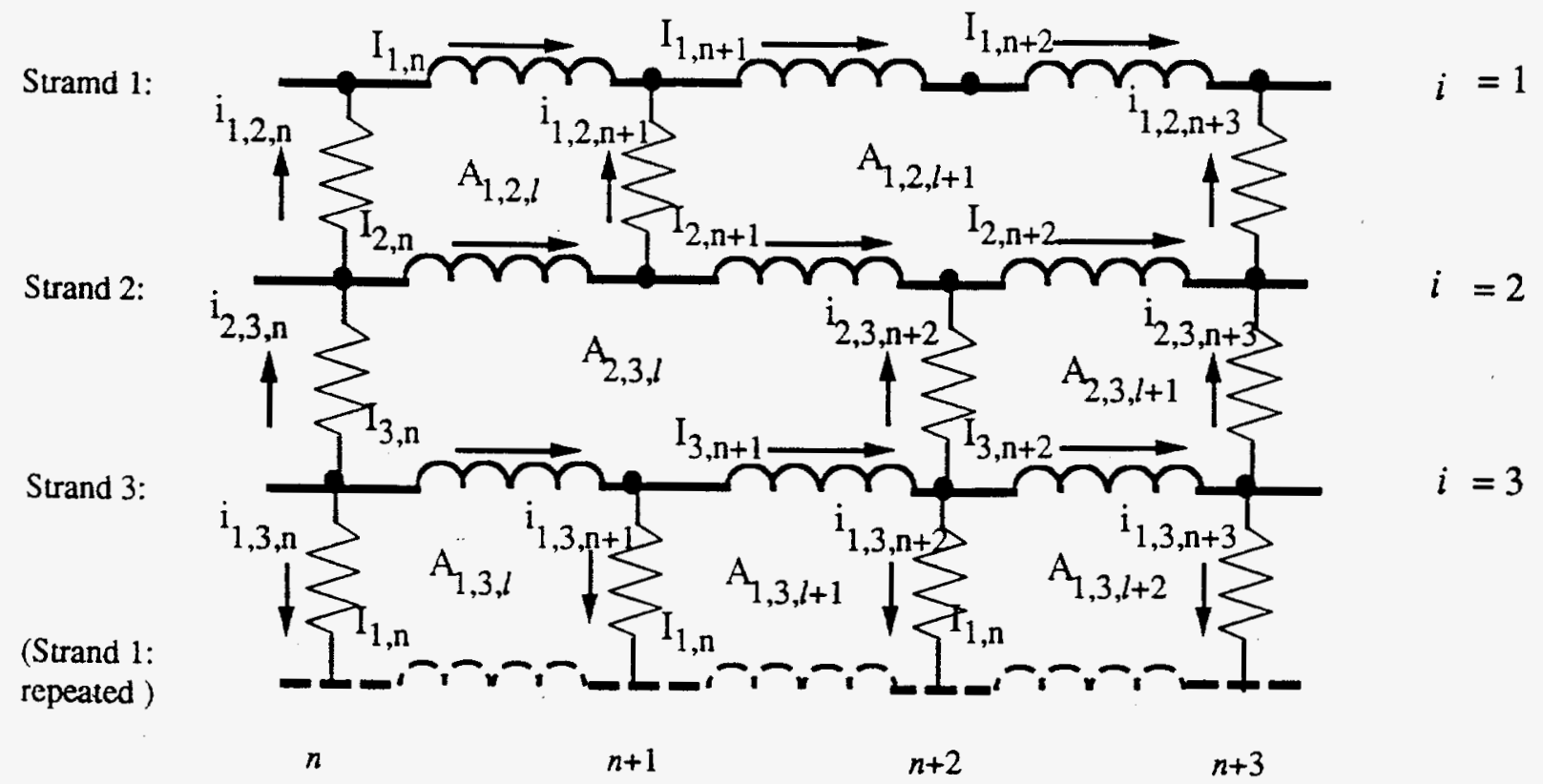

Figure 2.6 A section of the "Mesh Network Circuit" formed by three twisted strands with intermittent contacts. (The first strand is repeated at the bottom of the figure to facilitate the drawing.) The parallel and transverse currents (I 's and $i$ 's, respectively) are shown as they are defined in the text. The flux linkage areas (A's) are shown as if the strands were co-planar--the effects of twisting are difficult to show, but must be included in the solution. The resistors all have a value of $R 1$ and the inductors all have self-inductance L1. The mutual inductances (not shown) must be calculated from the geometry.

the contact current flowing from strand $j$ to strand $i$ at node $n$. By convention, the ordinal value of $i$ will always be less than $j$ to avoid directional ambiguity.

\section{Inductive Elements:}

The current branch connecting nodes of the same strand represents an ideally superconducting path. In reality, the resistive voltage drop across the length of superconductor is very small but not identically zero in non-zero electric and magnetic fields (Niessen [1993]). A superconducting current-voltage relation could be used to accurately model the resistive voltage drop but this level of detail has not been included in this model. Of course the model breaks down entirely for regimes in which the strands would be in the normal state.

With the zero resistance approximation, each segment of strand is modeled as an idealized inductor. The strands themselves are considered to 
have a round cross-section and within a strand the current density is assumed to be uniform. For the purposes of calculating inductances, the strand segments are considered to be parallel to the longitudinal axis of the cable. The self- and mutual-inductances of each strand segment can thus be calculated using inductance formulas from Grover (1946) which are given in Appendix A. The selfinductance of a single strand segment is found to be: $\mathrm{L} 1=2.0$ nano-Henry.

Computationally, it suffices to calculate mutuals only between strands with an axial separation of about five segment lengths or less. Although the mutual-inductances themselves do not vanish beyond the 5 segment limit, the differences in inductances do vanish, which is the important factor. The results presented later used a limit of seven segments to be conservative. In this thesis, the mutual-inductance between a segment of strand $i$ at axial position $n$ and a segment of strand $j$ at axial position $m$ is referred to as $M_{i, n, j, m}$. If $i=j$ and $m=n$, then $M_{i, n, j, m}$ is the self-inductance of the strand segment, L1.

Resistive Elements:

Where two strands come into contact, a finite contact resistance occurs. Although there are many "types" of contact (contact between strands within a triplet, between triplets, between bundles) and they are not necessarily uniform, each point of contact in the strand will be modeled by a simple resistor, $\mathrm{R} 1$, in the nominal case. Results will also be presented, however, for cases where the resistance will depend on the "type" of contact between strands.

Although the actual value of $\mathrm{R} 1$ will not be needed to solve the model if the results are non-dimensionalized, RI will be needed to obtain quantitative results. The trans-conductance measurements discussed above (Section 2.2.1) only measured the effective resistance between one strand and the rest of the cable over a given length, but an approximation of the local value R1 can be deduced from this result. The experimental measurement (conducted in DC 
conditions) calculates the transverse resistance from current and voltage readings. The current from the power supply travels into the single isolated strand, across the numerous contact points to the 26 other strands within the cable, and then returns to the power supply. Since the 26 strands are shorted together in the joint to the power supply bus bar, the circuit can be diagrammed as in Figure 2.7. The effective conductance, G, is thus simply the power supply current divided by the measured voltage and is equal to $N_{c} / R_{c}$ where $N_{c}$ is the number of contact points and $R_{c}$ is the resistance across each contact.

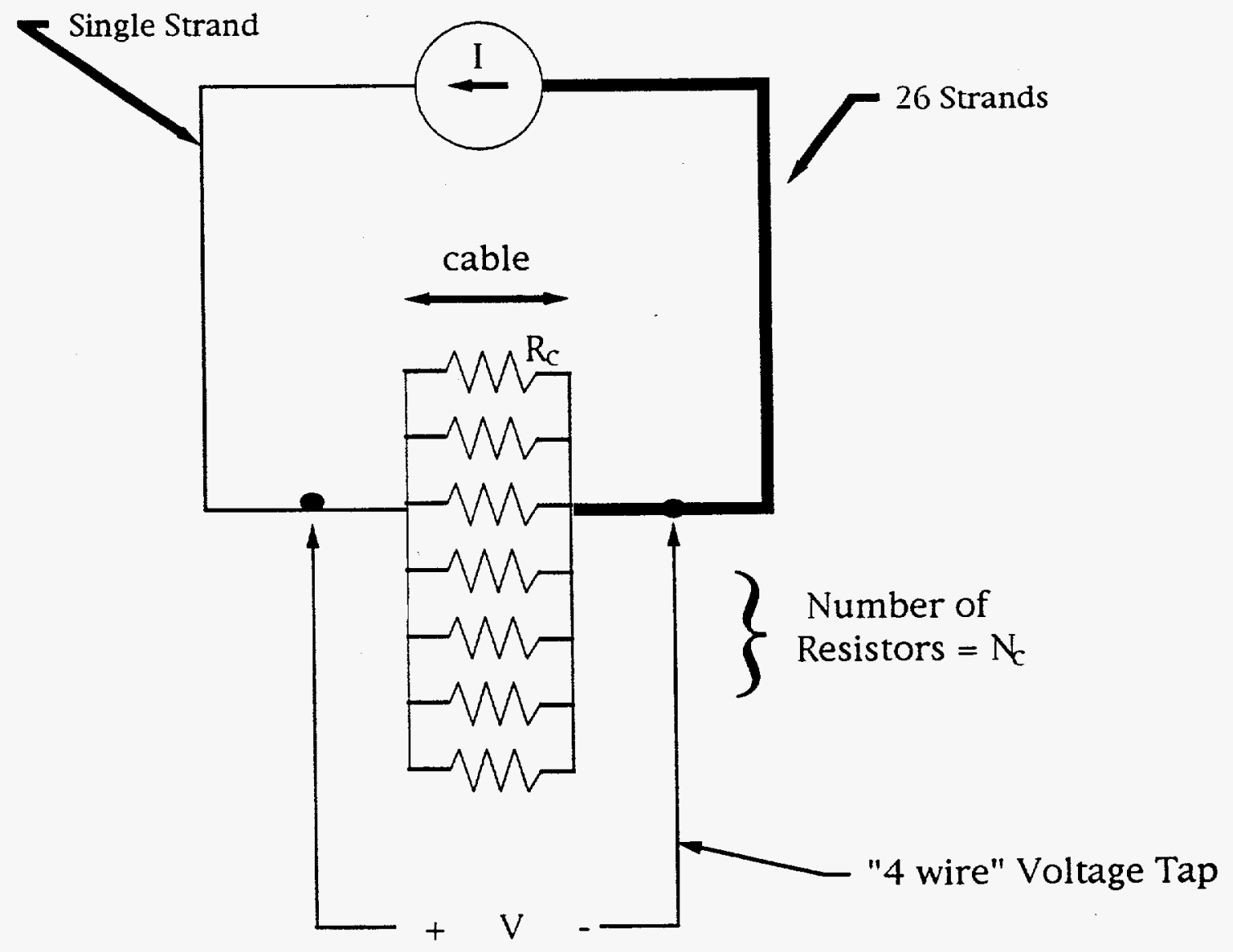

Figure 2.7 A schematic of the transverse resistance measurement used to establish the value of $R 1$ used in numerical analyses. See text for details. 
Although the actual value of $\mathrm{N}_{\mathrm{c}}$ is not known, the number of contacts which would occur in the model is known. For $1 \mathrm{~m}$ of model length (236 segment lengths), the average strand contacts the other 26 strands approximately 850 times. A relation for $\mathrm{R} 1$, the contact resistance in the model, which is consistent with the measured result is therefore: $(850 / R 1)=G$. Referring back to Eq. (2.2), this gives a value of:

$$
R 1=\frac{850}{G} \cong \frac{1}{2.5+0.25 B \cdot I} \quad \text { (ohms). }
$$

where $B$ is the background magnetic field in $\mathrm{T}$, and $I$ is the overall cable current in $\mathrm{kA}$. This is the value of R1 which will be used throughout the analysis. In most cases, an order of magnitude estimate will be sufficient to describe the general behavior of the cable and R1 will therefore frequently be given as a constant, $\mathrm{R} 1=0.1 \Omega$ (valid in the usual range of interest, $\mathrm{B} \sim 7 \mathrm{~T}, \mathrm{I} \sim 4 \mathrm{kA}$ ).

\section{Flux Linkages:}

The current loop formed by successive contacts points between a pair of strands is defined as a mesh. As shown in Figure 2.6, the current travels "up" one contact point, along one strand, "down" the next contact point, and back along the other strand to close the loop. These meshes can be determined from the geometry of the cable as approximated by the Mapped configurations. Any change in external flux which penetrates these meshes will cause an induced voltage around the mesh equal to rate of change of the field times the area of the mesh normal to the field direction. This voltage will be identified as $V_{i, j, l}$ in the circuit equations below where $i$ and $j$ represent the two strands involved and $l$ is an index which refers to the leftmost of the two contact points involved. $V_{i, j, l}=$ $\frac{d \bar{B}}{d t} \cdot A_{i, j, l} \hat{n}_{i, j, l}$ where the vector nature of the changing magnetic field is explicit and the orientation of the mesh area, $A_{i, j, l}$, is defined by its unit normal vector, $\hat{n}_{i, j, l}$. In Figure 2.6, all of the areas are shown with a unit normal vector out of the 
page for ease of presentation, but in the model the wires are twisted and normal vectors rotate accordingly. Over one period of length ( 48 segments) the sum of the individual flux linkages due to the uniform external magnetic field equals zero due tot the rotational symmetry of the geometry:

$$
\sum_{\text {alli,j,l }} \bar{B} \cdot A_{i, j, l} \hat{n}_{i, j, l}=0
$$

\section{Governing Equations:}

With the circuit elements and currents in the model well defined, it is possible to solve for the currents using Kirchoff's Current Law (KCL) and Kirchoff's Voltage Law (KVL). The resulting system of equations will depend on the boundary conditions and the time scales for which we wish to solve the model. In general, the number of variables will be quite large. In some limiting conditions, though, the equations can be greatly simplified. The different scenarios and their solution methods are described in the next section.

\subsection{Current Distribution in $\mathrm{CICC}$ for Several Scenarios}

The task of solving for the current distribution in a cable is best handled by dividing the problem into manageable pieces. The "divisions" considered in this thesis, outlined below, are distinguished by differences in forcing function (self- or external magnetic fields), length scale, and time scale. Because we have only employed linear circuit elements, the law of superposition is valid, and the current distributions due to separate effects can be added. For instance, it is possible to add current imbalances due to self-field effects to the current distribution due to external-field effects to obtain an overall current distribution. 


\section{Self-Field Cases:}

The effects of a linearly-ramping transport current on the overall distribution of current can be studied for two distinct length scales. First, for a periodic, infinitely long cable, the current distribution can be calculated for the entire time domain of the ramping event ${ }^{*}$. The distribution tends to uniformity as time advances. Second, for a cable of finite length with its ends terminated in specially designed joints, a separate analysis is needed. Although transients similar to those found in the periodic case also exist for finite non-periodic cables, the dominant current imbalances are not time-dependent. Thus, the approximate current distribution need be calculated only as a function of length. The effects of irregularities in the twist pitch can also be studied qualitatively.

\section{External-Field Cases:}

The effects of a linearly-ramping external-field are also different for infinitely long cables and those of finite length. In infinitely long, periodic cables, the current distribution can be calculated as a function of time. The solution for times much larger than a characteristic time constant can be found directly using much simpler means. In joint terminated cables of finite length, the current distribution can be found as a function of time in the limit that the joint resistance is much lower than the transverse resistance, as is the case for the cables being studied. The solution for this last case is also dependent on the length of the cable.

\subsubsection{Current Distribution in Infinitely Long, Periodic CICC}

Because the greatest mathematical differences arise between cases which are periodic and those which are finite, solutions for all of the periodic cases will

\footnotetext{
* Using the same calculations, the effects of other than linearly ramped driving functions could be studied for both the self field and external field analyses, but are not done so here.
} 
be presented before moving on to the finite scenarios. The infinite periodic case can be solved in more detail since we can take advantage of axial and rotational symmetries to reduce the number of unknowns. As in any periodic problem, the solutions found in the fundamental period are exactly repeated in the next fundamental period. This condition translates the infinite domain into a finite domain equal to one period in length with "periodic boundary conditions." The number of parallel currents in one 48 segment period is equal to the number of nodes, giving $48 * 27=1,296$ unknowns. A careful count of all the contact currents in one period of length adds another 2,304 unknowns $(6 * 42$ from each of Map A, C, E, and G, 6*51 from Maps B and F, and 6*57 from Maps D and H) to give a grand total of 3,600 unknowns. Fortunately, certain symmetries can be invoked which reduce the number of unknowns and thus the number of equations which must be solved.

\subsubsection{Self-Field, Time-Dependent Solution:}

The self-field effect is a time-dependent process which exists in a fully transposed cable due to the existence of a finite trans-conductance between the cabled strands. In these examples, the cabled strands begin at zero current when, at time $\mathrm{t}=0$, a linear ramping of the overall transport current, $I_{O}$, begins. The overall current ramp rate is $\dot{I}_{o}$. At time $\mathrm{t}=0^{+}$, the transport current distribution is governed entirely by the inductive couplings between the individual strand segments. Strand segments which are more loosely coupled (typically segments near the outside of the configuration) will carry more current than tightly coupled segments. The current in one segment of a strand can be dramatically different than the current in an adjacent segment of the same strand since the contact points between strands can be considered "shorted" for purposes of calculating this initial response to the step change in $\dot{I}_{o}$. As time t progresses, the resistive effects become important and currents will diffuse at a rate inversely 
proportional to the transverse resistance. Currents reach a uniform distribution on a time scale determined by a characteristic LR time-constant of the system, $\tau$.

Mathematical Model:

In order to solve for all of the parallel and transverse currents as a function of time, the complete resistive/inductive mesh network must be used. Since all of the currents are coupled, the full set of KCL and KVL equations must be solved simultaneously. Kirchoff's Current Law states that the sum of the currents entering a node is equal to the sum of the currents leaving the node. Kirchoff's Voltage Law states that the sum of inductive and resistive voltage drops around a closed mesh must equal the loop voltage generated by the time rate of change of the external magnetic flux through the mesh." These two laws can be stated mathematically as follows:

$$
\begin{aligned}
& \sum_{h} \mathbf{I}_{h}=0, \\
& \sum_{i} \mathbf{V}_{i}=-\dot{\Phi}
\end{aligned}
$$

where index $h$ indicates all of the currents connected to a certain node and index $i$ indicates every resistive or inductive element along a certain mesh.

For the periodic case, Kirchoff's Current Law is used at each of the nodes, node $_{i, n}$, and relates all of the transverse and parallel currents entering or leaving that node. Kirchoff's Voltage Law is used for each of the "meshes" and relates the inductive voltage drops along the parallel current segments to the resistive voltage drops across the contact points where transverse current flows. The sum of these voltage drops will equal zero since there is no external-field penetrating the mesh. The full set of these equations for the 27 strand cable become:

$\mathrm{KCL}: \quad I_{i, n}-I_{i, n-1}+\sum_{j=i+1}^{27} i_{j, i, n}-\sum_{j=1}^{i-1} i_{i, j, n}=0 ; i=1 . .27 ; n=1 . .48$

\footnotetext{
* Capacitance effects are not considered.
} 
KVL: $\quad \sum_{n=n_{i, j}^{\prime},}^{n_{i, j+k}^{\prime}} \sum_{m=n-7}^{n+7} \sum_{k=1}^{27}\left[M_{i, n, k, m} \cdot \dot{I}_{i, n}-M_{j, n, k, m} \cdot \dot{I}_{j, n}\right]+R 1\left(i_{i, j, n_{i, j, l}^{\prime}}-i_{i, j, n_{i, j, 1+1}^{\prime}}\right)=0$

$$
; i=1 . .27 ; j=i+1 . .27 ; l=1 . . . n c_{i, j}
$$

where the transverse currents $i_{i, j, n}$, the parallel currents $I_{i, n}$, and the mutualinductances $M_{i, n, j, m}$ appear as they are defined above. The indices $i$ and $j$ refer to the strand number; indices $n, k$, and $m$ refer to segment numbers; and $n_{i, j, l}^{\prime}$ refers to $l$-th contact point between strands $i$ and $j$. The number of contact points between each strand pair, $i$ and $j$, is $n c_{i, j}$, which ranges between 0 and 48 . The location of these contact points is determined from the mapped geometric strand configurations. Some further points to notice are that the subscripts on the transverse current in the first summation of Equations (2.8) reflect the convention that the first subscript is always less than the second to avoid directional ambiguity, and the factor of 7 in the second level summation of Equations (2.9) represents the spatial limit at which mutual coupling becomes unimportant, as explained above.

To account for the ramping transport current, the following boundary condition is imposed:

$$
\sum_{i=1}^{27} \dot{I}_{i, 1}=\dot{I}_{o}
$$

This single equation replaces one of the KCL Equations (2.8) in the governing system of equations.

Periodic Boundary conditions are invoked by setting $I_{i, N+n}=I_{i, n}$ and $i_{i, j, N+n}=i_{i, j, n}$ where $N=48=$ the period length. Thus the number of Equations (2.8) equals the total number of unknown parallel currents $(1,294)$ and the number of Equations (2.9) equals the number of unknown transverse currents $(2,304)$. While this system of equations is thus well-defined, the problem can be reduced to a smaller system without losing any information. 
The fundamental period of the self-field problem is 24 segments instead of the true spatial period of 48 segments. This is so because the self-field fluxes are determined by the relative positions of the strands and not the overall cable orientation. As was noted when introducing the geometric model of the cable (section 2.2.3), after 24 segments the strands return to the same relative orientation, even though the entire cable cross-section has been rotated $180^{\circ}$. Thus, the number of unknowns is first reduced by a factor of two.

By the same argument, the three-fold rotational symmetry apparent in the strand configurations mapped in Figure 2.2 reduces the number of unknowns by another factor of three. This symmetry can be seen by following, for example, strands 1, 14, and 27 as they progress through the strand configurations. In each Map, A-H, they lie at the vertices of an equilateral triangle centered at the midpoint of a "triangularly symmetric" cross-section. The three strands (each in different bundles) are therefore "equivalent" and it is only necessary to keep one of them as an unknown. The currents in strands 10-27 can thus be equated to their rotationally symmetric counter-part amongst strands 1-9.

Another symmetry present in the cable, is also most easily shown by example. Strand 7 in Map A at first seems unrelated to strand 1. According to Table 1, however, the strand in the position labeled "7" in Map H next goes to the position labeled " 1 " in Map A. In this way the path of strand 7 is actually the same as the path of strand 1, but shifted by eight segments. Similarly, the path of strand 4 is also the same as the path of strand 1, but is shifted by 16 segments. Strands 5 and 8 are likewise related to strand 2 as are strands 6 and 9 to strand 3 . Thus, the number of unknowns is reduced by another factor of three and the total number of unknowns has been reduced by a factor of 18 , leaving 200 currents for which to solve. The correspondence between strands eliminated due to symmetry and the remaining "primary" strands $(1,2$, and 3$)$ is shown in Table 2. 


\begin{tabular}{|c|c|c|}
\hline$A$ & $B$ & $C$ \\
\hline 1 & 1 & 0 \\
\hline 2 & 2 & 0 \\
\hline 3 & 3 & 0 \\
\hline 4 & 1 & 8 \\
\hline 5 & 2 & 8 \\
\hline 6 & 3 & 8 \\
\hline 7 & 1 & 16 \\
\hline 8 & 2 & 16 \\
\hline 9 & 3 & 16 \\
\hline
\end{tabular}

\begin{tabular}{|c|c|c|}
\hline$A$ & $B$ & $C$ \\
\hline 10 & 3 & 16 \\
\hline 11 & 1 & 16 \\
\hline 12 & 2 & 16 \\
\hline 13 & 3 & 0 \\
\hline 14 & 1 & 0 \\
\hline 15 & 2 & 0 \\
\hline 16 & 3 & 8 \\
\hline 17 & 1 & 8 \\
\hline 18 & 2 & 8 \\
\hline
\end{tabular}

\begin{tabular}{||c|c|c|}
\hline$A$ & $B$ & $C$ \\
\hline 19 & 2 & 8 \\
\hline 20 & 3 & 8 \\
\hline 21 & 1 & 8 \\
\hline 22 & 2 & 16 \\
\hline 23 & 3 & 16 \\
\hline 24 & 1 & 16 \\
\hline 25 & 2 & 0 \\
\hline 26 & 3 & 0 \\
\hline 27 & 1 & 0 \\
\hline
\end{tabular}

Table 2. For the self-field case, the redundant strand in column $A$ is equivalent to the "Primary" strand in column B but shifted by the number of segments shown in column C. See text for a complete explanation.

The reduction of the number of unknowns from 3600 to 200 will make the use of traditional matrix equation solution techniques much easier. For a nonperiodic strand in which symmetric reductions were not possible, more advanced techniques would be necessary. Even if the problem could be solved directly for the original number of unknowns, the 18 fold reduction lowers the CPU memory and time requirements by a factor of approximately $324\left(=18^{2}\right)$ for the full problem (Press, et al. [1989]).

Equations (2.8), (2.9), and (2.10) represent a coupled system of first order differential equations which will have to be solved numerically. In this thesis, a simple finite difference technique is used employing a forward Euler scheme. Although this scheme is rarely recommended for most applications since it is often unstable and inaccurate (Strang [1986]), it is appropriate for this problem because of the inherent stability of the system (as was discovered rather than calculated). The forward Euler scheme replaces the time derivative of a function by a simple finite difference. For a time-step size, $\Delta t$, the relation is:

$$
\dot{y}_{t}=\left(y_{t+1}-y_{t}\right) / \Delta t
$$

where subscript $t$ represents the current time step. The stability and accuracy of the technique will be demonstrated later. 
Forward Euler finite differencing linearizes the first order differential Equations (2.9) and, together with Equations (2.8), a simple matrix equation is formed: $\mathbf{A} \cdot \mathbf{x}=\mathbf{b}$. The equations can thus be solved using traditional linear algebra techniques to give the transverse and parallel currents at each time step. A FORTRAN program written to perform this task relies on the packaged routines LFCRG and LFIRG provided by IMSL (1987). The LFCRG routine needs to be called only once to calculate the LU decomposition of the coefficient matrix (Press, et al., [1989]) which is then used by LFIRG at each time step to calculate the unknown current vector. The "iterative refinement" techniques employed by LFIRG are necessary because the equations are "algorithmically singular"--a condition where very small changes in the coefficient matrix, A, cause large changes in the solution vector, $x$ (IMSL [1987]). Even on a CRAY computer ${ }^{*}$ the iterative process consumes a lot of CPU time, on the order of 1 minute per time step. At this point, the factor of $18^{2}$ reduction in computation time achieved through symmetry arguments can be greatly appreciated. It is also fortunate that the time step size, $\Delta t$, is practically unconstrained so that an accurate solution at any time could be calculated in a minimum of time steps. This will be demonstrated later.

\section{Results:}

To maintain generality, dimensionless variables will be used, where possible. The results are shown in Figure 2.8 for several dimensionless times as separate graphs of normalized current vs. axial position for the three independent "primary" strands. The domain of axial positions covers the 24 segments of the fundamental period of the self-field problem; the segment length is $4.2 \mathrm{~mm}$. The currents are normalized by $\dot{I}_{0} \mathrm{t} / 27$, the amount of current which would be carried in each strand if the distribution were uniform, where $t$ is the

\footnotetext{
* The Cray C-90 at the National Energy Research Super Computer (NERSC) facility was used.
} 

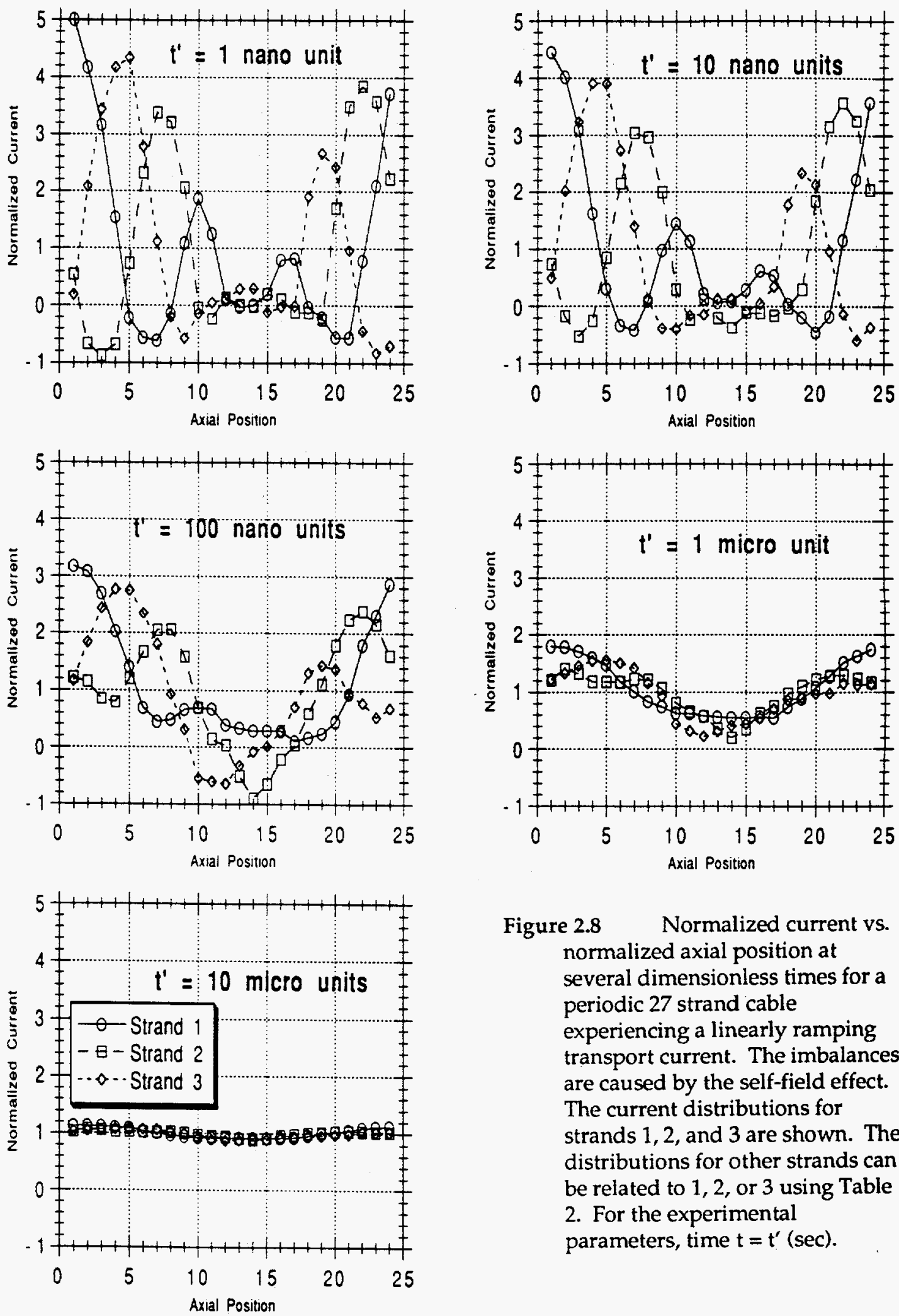

Figure 2.8 Normalized current vs. normalized axial position at several dimensionless times for a periodic 27 strand cable experiencing a linearly ramping transport current. The imbalances are caused by the self-field effect. The current distributions for strands 1,2, and 3 are shown. The distributions for other strands can be related to 1,2 , or 3 using Table 2. For the experimental parameters, time $t=t^{\prime}(\mathrm{sec})$. 
time. For each graph the true time is equal to the dimensionless time, $t^{\prime}$, divided by a multiple of the contact resistance: $t=t^{\prime} /(10 * R 1)$, where $t$ is in seconds and $R 1$ in $\Omega$. For a nominal measured value of contact resistance, $R 1=0.1 \Omega$, time $t=$ $t^{\prime}(\mathrm{sec})$.

It should be remembered that the currents in each of the 24 strands not shown in the figure can be directly related to the currents in one of the strands shown using Table 2. The solutions for any strand is either "coincident" with one of the traces given, or "phase shifted" in position by either 8 or 16 segments. If it were possible to clearly show traces of each strand in one figure, it would be more obvious that the average of all the strand currents at each position equals 1 .

The transverse currents are also found in the solution of the problem. While these currents would be important in calculating the AC loss of the cable, they do not affect the stability of the cable as it has been defined above. Since these models are being developed primarily to look at stability (for now, at least), the transverse currents will not be presented at this time. However, it should be noted that this model does provide a means for studying inter-strand AC losses* .

For times less than $\mathrm{t}^{\prime} \sim 10^{-6}$, significant current imbalances can be seen to exist in the cable. During this period, the currents are diffusing between the strands through the transconductive contact points as they relax from the initial distribution generated by the step change in current derivative. Although the imbalances are severe--each strand even exhibits "counter-currents" at some positions--the actual magnitudes of the currents are small since the total current in the cable has only just begun to increase from zero, $I_{O}=\mathrm{t} * \dot{I}_{o}$. For instance, for $R 1=0.1 \Omega$, and a typical current ramp rate of $1 \mathrm{kA} / \mathrm{s}$, typical of values in the experiment, the total current in the cable is approximately $10 \mathrm{~mA}$ when the currents stabilize to a uniform value at time $t \sim 10 \mu$ s. Since the current carrying

\footnotetext{
* Losses for different transport current wave forms and frequencies could also be studied.
} 
capacity of each of the strands is on the order $100 \mathrm{~A}$, the self-field effect cannot be considered a source of instability in a fully periodic cable for ramp times much greater than the characteristic LR time constant, estimated from the results to be $\sim 100$ nano-seconds for the 27 strand cable.

For the periodic case, the equilibrium current distribution is uniform. The ability to achieve this solution numerically even for large time steps is a confirmation of the accuracy and stability of the finite differencing scheme employed. A more convincing demonstration of the accuracy and stability is given for the external-field case.

\subsubsection{External-Field, Time-Dependent Solution:}

In the external-field case, a uniform background field is ramped from zero at a constant rate, $\dot{B}$, beginning at time $\mathrm{t}=0$. Initially, there is no net transport current and no circulating currents in the cable. At the start of the ramp, a step change in loop voltage is induced around the meshes defined by the model cable geometry above. At time $t=0^{+}$, the inductive elements in the mesh network determine the initial current distributions in each strand. As time progresses, the mesh currents evolve into their steady-state values as determined by the resistive elements of the mesh. Unlike the self-field case, the currents will not be uniform in the steady-state. It bears repeating that the calculated distributions for this zero transport current case could be superimposed on the self-field distribution caused by a ramping transport current.

The full transient solution will be presented first and then a simplified solution method which is accurate for times sufficiently greater than the characteristic time constant of the problem will be presented. Not only is the simplified version a more useful tool for analyzing larger and more complex cables, it also validates the stability and accuracy of the transient solution. 


\section{Mathematical Model:}

As in the self-field case Kirchoff's Current Law will be employed at each of the nodes and Kirchoff's Voltage Law will be applied to each of the meshes. However, the ramping external-field contributes an additional parameter to the problem, the induced loop voltage, $V_{i, j, l}$, as it was defined above. With this exception the governing equations are the same as above but are repeated here:

$\mathrm{KCL}: I_{i, n}-I_{i, n-1}+\sum_{j=i+1}^{27} i_{j, i, n}-\sum_{j=1}^{i-1} i_{i, j, n}=0 ; i=1 . .27 ; n=1 \ldots 48$

$\mathrm{KVL}: \sum_{n=n_{i, j,}^{\prime}}^{n_{i, j, k+1}^{\prime}} \sum_{m=n-7}^{n+7} \sum_{k=1}^{27}\left[M_{i, n, k, m} \dot{I}_{i, n}-M_{j, n, k, m} \dot{I}_{j, n}\right]+R 1\left(i_{i, j, n_{i, j,}^{\prime}}-i_{i, j, n_{i, j, 1}^{\prime}}\right)=V_{i, j, l}$

$$
; i=1 . .27 ; j=i+1 . .27 ; l=1 \ldots n c_{i, j}
$$

The periodic boundary conditions are also the same as mentioned above and there are again 3600 unknowns and an equal number of equations.

To account for the lack of transport current, the following boundary condition is imposed:

$$
\sum_{i=1}^{n} I_{i, 1}=0
$$

This single equation replaces one of the KCL Equations (2.12) in the governing system of equations.

The symmetry of the cable is again exploited to reduce the number of variables to a reasonable quantity. The first reduction takes advantage of the fact that the strand configuration at one point of the cable is repeated a half period length away with the overall orientation rotated by $180^{\circ}$. Because the externalfield points in a constant direction, the flux linkages at the two locations will be equal and opposite. This being the only difference, it follows that the parallel and transverse currents at one location will also be equal and opposite to those found a half period length away. Stated formally: 


$$
I_{i, N / 2+n}=-I_{i, n} \quad \text { and } \quad i_{i, j / 2+n}=-i_{i, j, n}
$$

where $N=48=$ the period length. These relations reduce the number of unknowns by a factor of two.

A further reduction is possible by noting the equivalency of certain strands. There are not as many equivalencies as were found in the self-field case because the presence of the external-field destroys the rotational symmetry of the problem. Nonetheless, the repetitive nature of the strand configurations over the length of the period does provide a three-fold reduction. This can be seen by following the strand that begins at the first segment in position 21 of Map A. Using the steps defined above to follow the path of this strand, at the 17th segment the strand is found to be in position 27 of Map A. At this point it is critical to remember that Map A must be rotated counter-clockwise by $120^{\circ}$ to obtain the correct orientation of the cable with respect to the field at segment 17. In doing so, it is seen that strand position 27 at segment 17 is equivalent to position 1 at segment 1 . Thus, strand 21 is equivalent to strand 1 , only shifted by 16 segments. Similarly the currents in strands $10-27$ can be equated to corresponding currents in strands 1-9, achieving the desired three-fold reduction. Table 3 shows the strand equivalencies for the external-field case.

There are 600 remaining unknowns for which to solve. The same numerical techniques employed above are again applicable. "Iterative refinement" was required and the solution consumed approximately 1 minute of CPU time per time step on the Cray.

\section{Results:}

To keep the results general, dimensionless variables are used where possible. The results are shown for several dimensionless times as separate graphs of normalized current vs. axial position for three of the nine independent strands, including the 


\begin{tabular}{|c|c|c|}
\hline$A$ & $B$ & $C$ \\
\hline \hline 1 & 1 & 0 \\
\hline 2 & 2 & 0 \\
\hline 3 & 3 & 0 \\
\hline 4 & 4 & 0 \\
\hline 5 & 5 & 0 \\
\hline 6 & 6 & 0 \\
\hline 7 & 7 & 0 \\
\hline 8 & 8 & 0 \\
\hline 9 & 9 & 0 \\
\hline
\end{tabular}

\begin{tabular}{||c|c|c|}
\hline$A$ & $B$ & $C$ \\
\hline 10 & 3 & 16 \\
\hline 11 & 1 & 16 \\
\hline 12 & 2 & 16 \\
\hline 13 & 6 & 16 \\
\hline 14 & 4 & 16 \\
\hline 15 & 5 & 16 \\
\hline 16 & 9 & 16 \\
\hline 17 & 7 & 16 \\
\hline 18 & 8 & 16 \\
\hline
\end{tabular}

\begin{tabular}{||c|c|c|}
\hline$A$ & $B$ & $C$ \\
\hline \hline 19 & 2 & 32 \\
\hline 20 & 3 & 32 \\
\hline 21 & 1 & 32 \\
\hline 22 & 5 & 32 \\
\hline 23 & 6 & 32 \\
\hline 24 & 4 & 32 \\
\hline 25 & 8 & 32 \\
\hline 26 & 9 & 32 \\
\hline 27 & 7 & 32 \\
\hline
\end{tabular}

Table 3. For the external-field case, the redundant strand in column $A$ is equivalent to the "Primary" strand in column B but shifted by the number of segments shown in column C. See text for a complete explanation.

strand with the maximum induced current, in Figure 2.9.. The asymptotic results for all nine independent strands are shown in Figure 2.10 for the sake of completeness. Currents in strands not shown can be related to the strands shown using Table 23 The currents are normalized by the quantity $\dot{B} /(10,000 \mathrm{R} 1)$, the rate of change of the field divided by a multiple of the nominal contact resistance. The true time in seconds is proportional to the dimensionless time divided by the contact resistance in ohms: $t=t^{\prime} /\left(10^{*} R 1\right)$ (sec). For $R 1=0.1 \Omega$, the nominal contact resistance, $t=t^{\prime}$.

The maximum imbalances occur in the steady-state distribution and thus any stability implications would be greatest at the end of a ramping event when the transport current would be highest and the magnetically induced imbalances could bring certain strands closest to their limiting currents in certain locations. However, the maximum induced current encountered is an overcurrent on the order of $4 * \dot{B} /(10,000 * \mathrm{R} 1)$ amps. For a typical ramp of $1 \mathrm{~T} / \mathrm{s}$ and the nominally measured value of $R 1=0.1 \Omega$, this maximum current is approximately $4 \mathrm{~mA}$. Compared to the normal operating current of a strand which is on the order of $100 \mathrm{~A}$, the induced currents do not seem significant in terms of stability analysis. 

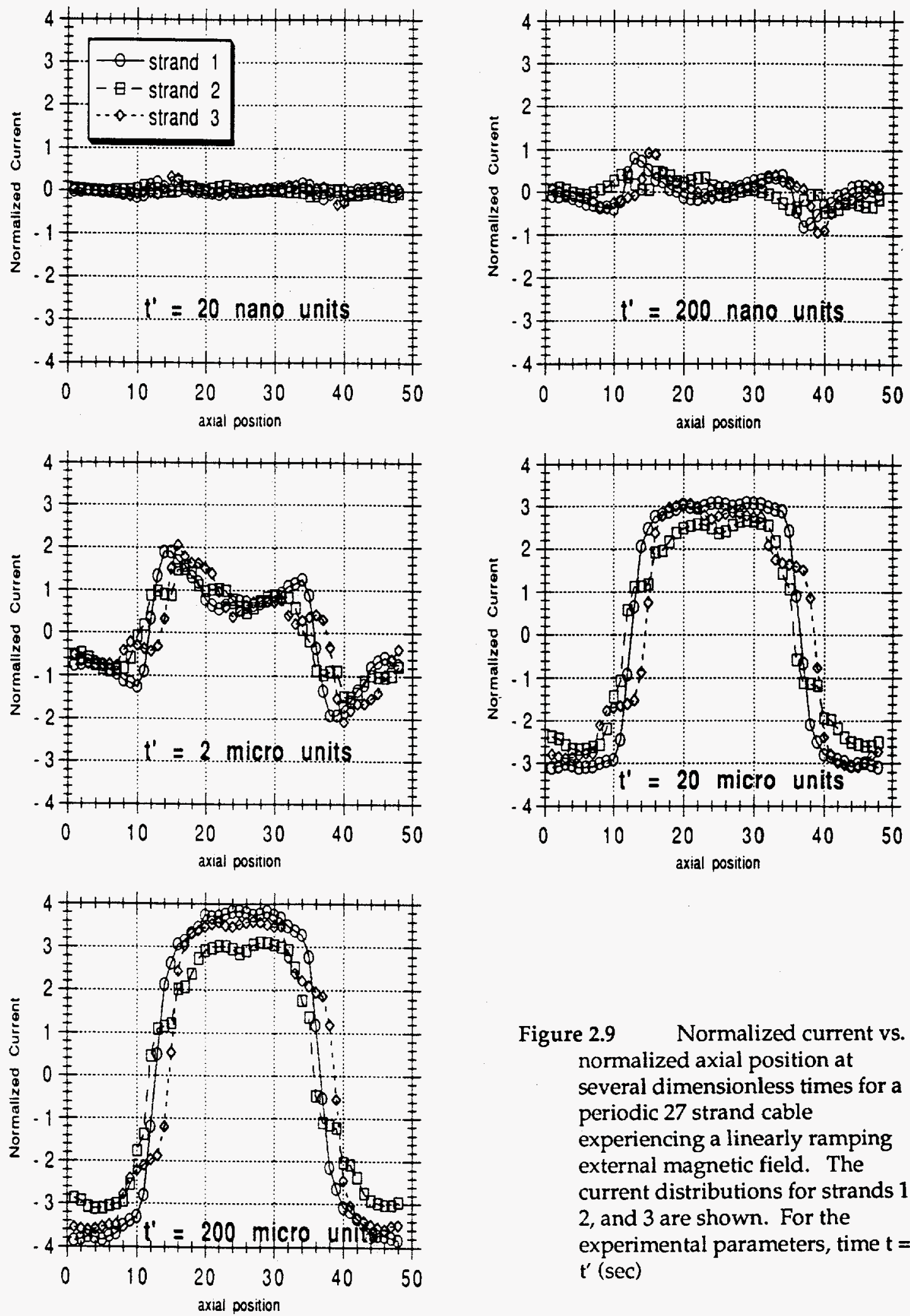

Figure 2.9 Normalized current vs. normalized axial position at several dimensionless times for a periodic 27 strand cable experiencing a linearly ramping external magnetic field. The current distributions for strands 1 , 2 , and 3 are shown. For the experimental parameters, time $\mathrm{t}=$ $t^{\prime}(\mathrm{sec})$ 


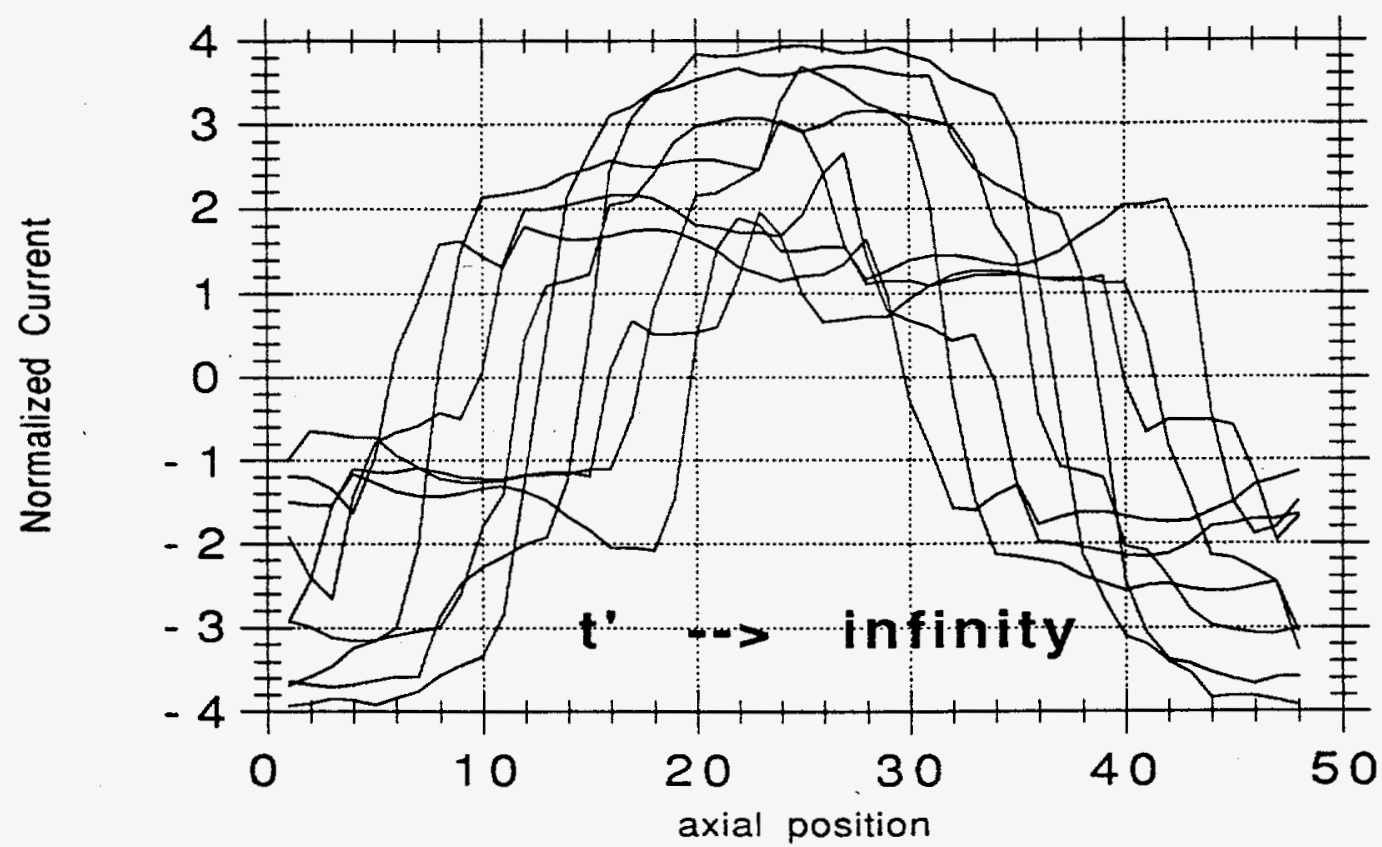

Figure 2.10 Normalized current vs. normalized axial position as time approaches infinity for a periodic 27 strand cable experiencing a linearly ramping external magnetic field. The current distributions for strands 1 through 9 are shown. The distributions for other strands can be related to 1 through 9 using Table 3 . 
Again, the transverse currents are not presented but would form the basis for a future study of inter-strand $\mathrm{AC}$ loss due to transverse external-field changes. This is a potentially more useful study for the case of periodic cables, but is not covered in this thesis.

The results show that the induced currents reach their steady-state distribution on the dimensionless time scale of $\mathbf{t}^{\prime} \sim 10,000$. A dimensionless characteristic time constant of the problem is on the order of $\tau^{\prime} \sim 1,000$. For the nominal value of contact resistance measured in the cable, this translates into a time constant $\tau \sim 10 \mu \mathrm{s}$. The steady-state distribution is achieved almost immediately. For this reason, a simplified solution technique which directly calculates the steady-state distribution has been developed.

\subsubsection{External-Field, Steady-State Solution:}

A method of solution which directly finds the steady-state current distribution in a CICC experiencing a linearly-ramping external magnetic field offers one main advantage: a savings in computational expense. As was mentioned above, using the transient analysis presented above to estimate the steady-state response requires appreciable CPU time for the 27 strand sample analyzed here. Using the same procedure for cables with more strands and/or less symmetry could be prohibitively expensive in terms of CPU time and memory requirements. Since the steady-state is reached very quickly for cables with high inter-strand resistances, a method which directly finds the steady-state solution can be a more useful tool than the full transient analysis. The direct steady-state solution can also be used to verify the stability and accuracy of the numerical techniques employed in the transient analysis.

\section{Mathematical Model:}

In the steady-state limit, the current distribution has stabilized and thus there are no inductive effects caused by changing currents. The model of the 


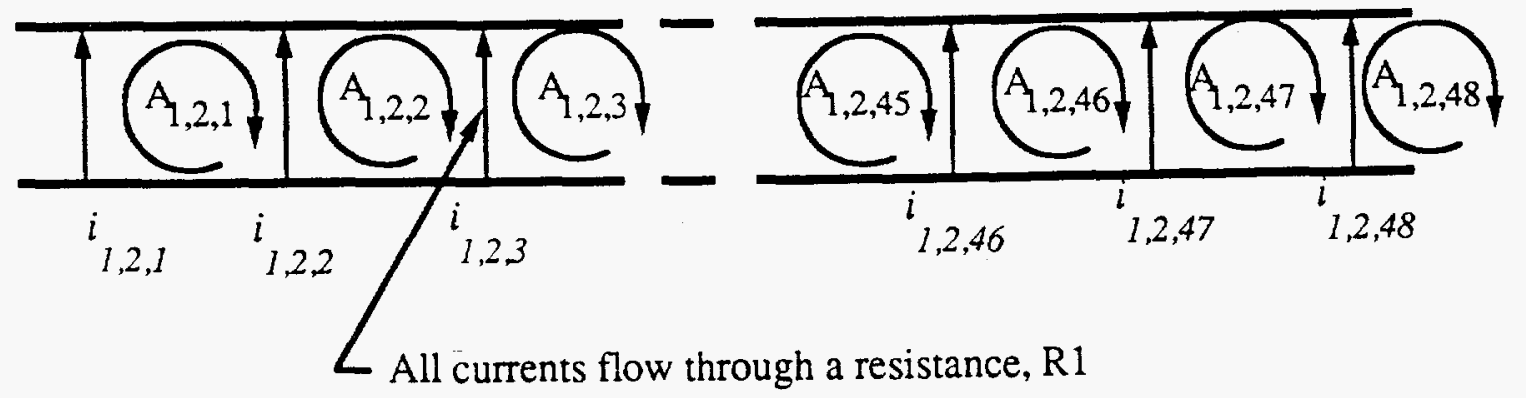

Figure 2.11 The transverse currents between strands 1 and 2 can be solved indepently from other currents in the cable. The necessary KVL equations are indicated by "loops" in the figure. Periodic boundary conditions are used to give an equal number of equations as unknowns.

problem in this limit would be the same as that shown in Figure 2.6, with the inductors replaced by non-inductive, superconducting paths. Although Kirchoff's Current and Voltage Laws are again used to solve the resulting mesh network, the KCL equations are no longer coupled to the KVL equations. This is true because only the transverse currents experience any voltage drop and thus the parallel currents drop out of the KVL equations.

Furthermore, the KVL equations themselves can be broken into subsets which can be solved indepently. The transverse currents flowing between two strands along the length of the cable are independent of the transverse currents flowing between any other strands. This concept is illustrated in Figure 2.11, which schematically shows the transverse currents which flow between strands one and two at the contact points found from the model of the 27 strand cable presented above. Each current loop which exists between the points of contact is again called a mesh and Kirchoff's Voltage Law can be applied to each: 


$$
\text { KVL: } \quad R 1\left(i_{1,2, l}-i_{1,2, l+1}\right)=V_{1,2, l} \quad ; l=1 \ldots 48
$$

where the transverse currents and the induced loop voltage, $V_{1,2, l}$, are defined in the same manner as above. Using periodic boundary conditions, $i_{1,2,49}=i_{1,2,1}$, and thus there are an equal number of equations as unknown transverse currents. The transverse currents between any two strands can thus be found from Equations (2.16) and are independent of the other currents in the problem.

Equations (2.16), however, form a singular or degenerate set of equations-the determinant of the associated coefficient matrix equals 0 . In order to solve the set of equations, it is necessary to replace one of the equations with an appropriate constraint on the variables. In this case, the constraint arises from the geometry of the problem. Since the orientation of the cable with respect to the field rotates $180^{\circ}$ every half period, as explained above, the symmetry conditions stated in Eq. (2.15) again apply and can be re-written as:

$$
\begin{aligned}
& \sum_{\text {all }} i_{i, j, l}=0 \\
& \sum_{\text {all } n} I_{i, n}=0
\end{aligned}
$$

Equation (2.17) provides the necessary constraint for Equations (2.16) and allows a direct solution for the transverse currents between strands one and two using standard linear algebra techniques. The same procedure is then used to find the transverse currents between the other strand pairs.

After solving for all of the transverse currents, Kirchoff's Current Law is used to solve for the parallel currents. The KCL equations also do not need to be solved all at once. The parallel currents in strand one, for example, can be determined from the subset:

$\mathrm{KCL}: \quad I_{i, n+1}-I_{i, n}+\sum_{k=1}^{i-1} i_{k, i, n}-\sum_{k=i+1}^{27} i_{i, k, n}=0 ; n=1 . . .48$

where subscript $i$ equals 1 . Remembering the convention that transverse current, $i_{i, j, n}$, is defined to flow from strand $j$ to strand $i$ where $i<j$, the first 
summation represents transverse currents leaving node $i, n$ and the second summation represents transverse currents entering node $i, n$. The periodic boundary conditions give $I_{1,49}=I_{1,1}$ and since all of the transverse currents in the summations are known, the number of unknown parallel currents is equal to the number of equations. The parallel currents along strand one can thus be found from Equations (2.19) and are independent of the parallel currents in the other strands.

Equations (2.19) are again singular, however, and a constraint on the variables must be employed. When the symmetry condition expressed in Eq. (2.18) is used to replace one of the equations from the set of Eq.'s (2.19), the problem is well defined and the parallel currents along each segment of strand one can be solved. The same technique is then used to solve for the currents along the other strands.

For the periodic problem, it is thus possible to directly solve for the steady-state current distribution due to a linearly-ramping external-field with a minimum of computational difficulty. The matrix equations, $\mathbf{A} \cdot \mathbf{x}=\mathbf{b}$, implied by the properly constrained sets of Equations (2.16) and (2.19) are similar to each other since both have a coefficient matrix of the type:

$$
\mathbf{A}=\left[\begin{array}{ccccc}
a & -b & & & \\
& b & -c & & \\
& & c & -d & \\
& & & d & -e \\
1 & 1 & 1 & 1 & 1
\end{array}\right]
$$

For such a coefficient matrix, the solution vector $x$ can be found in $2 \mathrm{~N}$ steps rather than the usual $\mathrm{N}^{2}$ steps needed for general matrix equations of order $\mathrm{N}$ (Press, et al. [1989]). The entire solution for the steady-state current distribution is thus quite speedy since the problem is broken into a series of smaller matrix 
equations, each of a form which can be solved efficiently. For the 27 strand sample, the solution is achieved in fractions of a CPU-second on the Cray.

Results:

The calculated results for the steady-state current distribution in the cable are shown in Figure 2.12 for the nine strands presented in Figure 2.10, to which the results should be compared. The currents are again normalized by the same scaling factor $\dot{B} /(10,000 \mathrm{R} 1)$ and they generally corroborate the previous results which represent the asymptotic limit of the transient analysis. A more direct comparison is given in Figure 2.13 where the current profiles for strands one and nine are given both as they were calculated using the direct steady-state solution and as they were calculated using the transient solution above with a final time of $100 \mu \mathrm{s}$ reached in two time steps. The ability of the transient solver to asymptotically match the expected results with only small discrepancies, as seen in the figure, empirically verifies the stability and accuracy of the transient solution technique.

The study of self- and external-field effects on infinite periodic conductors have so far considered each of the contact points in the cable to exhibit the same resistance, R1. At this point, the contact resistance will be differentiated by the type of contact between strands, allowing for three distinct possibilities: the contact resistance between two strands within the same triplet will be called $R_{t}$; the contact resistance between two strands in different triplets but the same bundle will be called $R_{b}$; and finally, the contact resistance between two strands in different bundles will be called $R_{c}$. $\quad$ The relative values of $R_{t}, R_{b}$, and $R_{c}$ are unknown but for the purposes of a simplified analysis the following relationship is used:

* The effects of a stochastically varying transverse resistance have been studied for a two strand model in Niessen, van Damme (1993). 


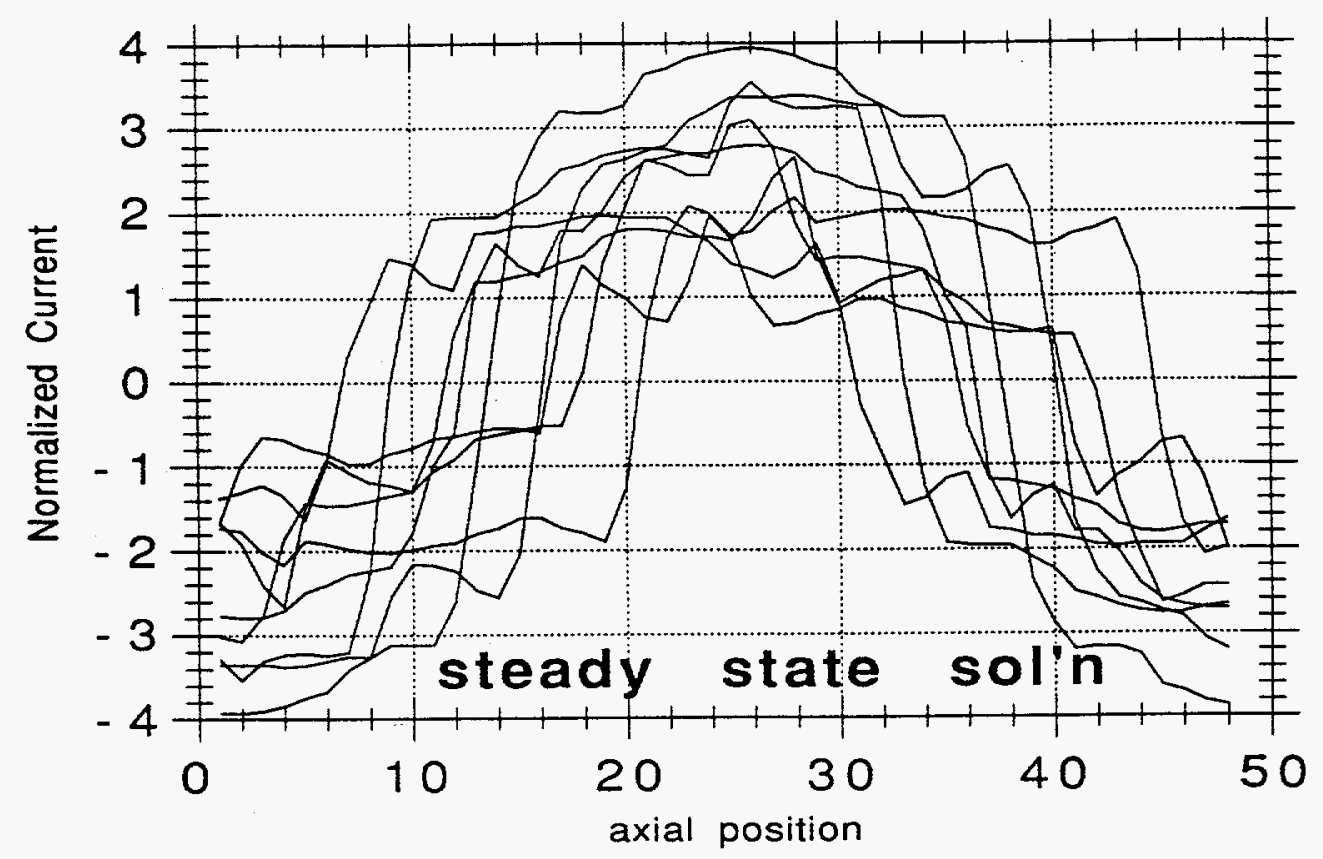

Figure 2.12 Normalized current vs. normalized axial position in the steady state limit for a periodic 27 strand cable experiencing a linearly ramping external magnetic field. The current distributions for strands 1 through 9 are shown. The distributions for other strands can be related to 1 through 9 using Table 3 .

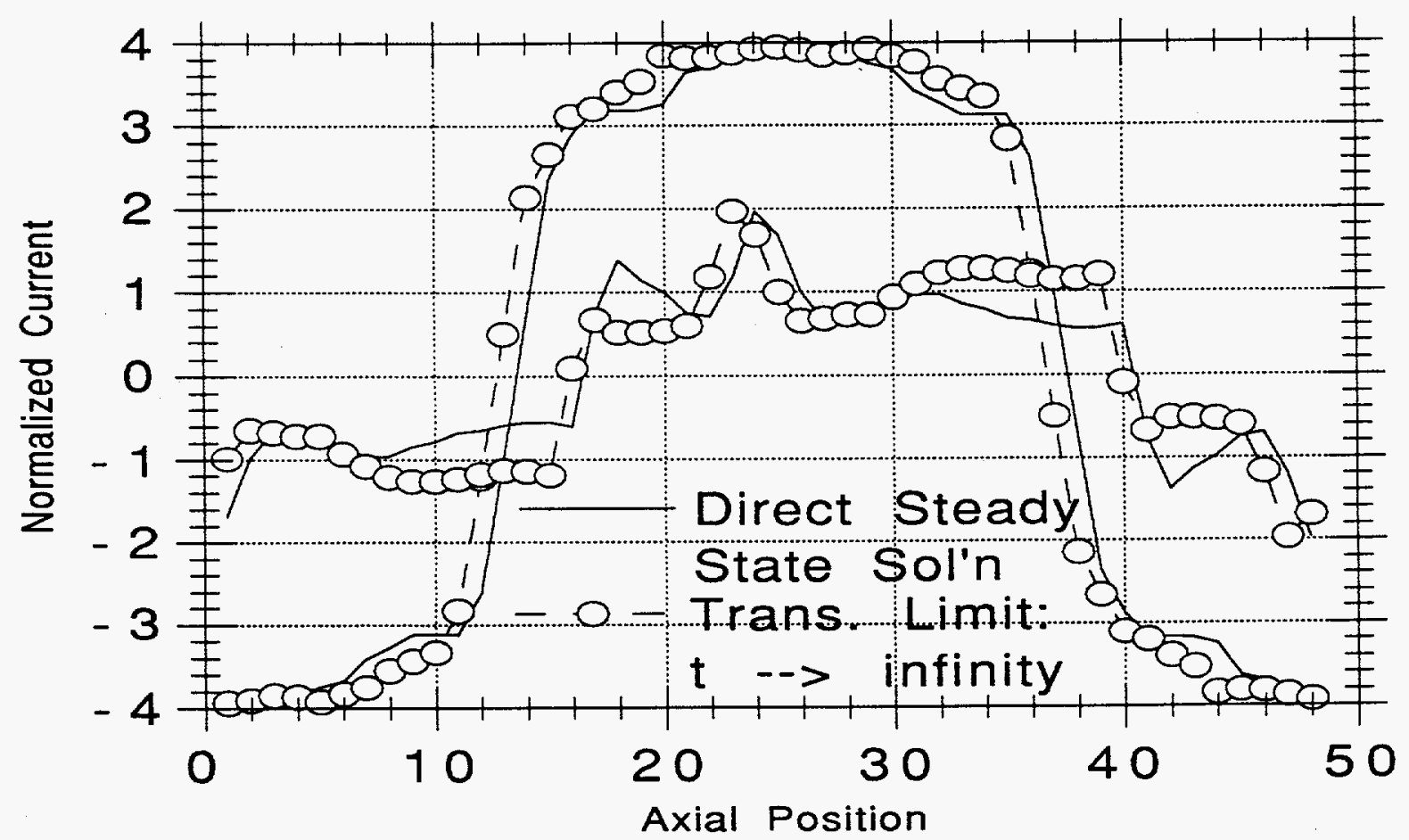

Figure 2.13 Comparison of the direct steady state solution and the asymptotically approached limits of the transient analysis for a ramping external field. 


$$
\mathrm{R}_{\mathrm{c}}=2^{f} \mathrm{R}_{\mathrm{b}}=2^{f} \mathrm{R}_{\mathrm{t}}
$$

where the power index, $f$, is an arbitrarily defined "doubling factor." For a doubling factor of zero, $R_{t}=R_{b}=R_{c}$; for a doubling factor less than zero, $R_{t}>R_{b}>$ $R_{c}$; and for a doubling factor greater than zero, $R_{t}<R_{b}<R_{c}$. In any case, the effective conductance between one strand and the 26 other strands in the cable is set equal to the experimentally measured value (Equation (2.2)). Using an equation similar to Eq. (2.5) and the number of each type of contact as found from the geometric model, consistent values for $R_{t}, R_{b}$, and $R_{c}$ can be found.

The effects of approximating the strand resistances in this way are shown in Figure 2.14. The results are given as the maximum induced steady-state parallel current anywhere in the cable normalized by $\dot{B}$, vs. the doubling factor, $f$. The single point at which the doubling factor equals zero corresponds to the

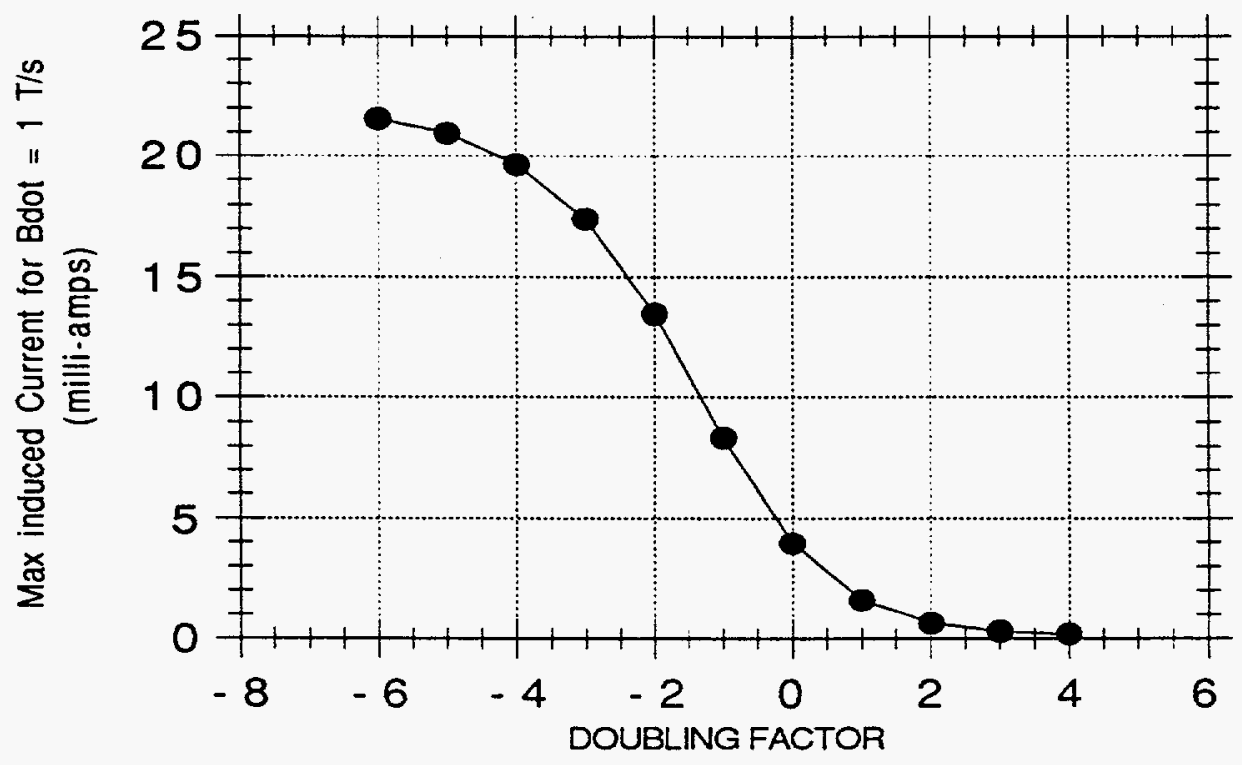

Figure 2.14 Maximum induced current in the cable vs. "doubling factor" for ramping external fields. See text for explanation. 
uniform contact resistance case considered previously. The figure suggests higher induced currents would result for negative doubling factors, when the ordering of the different contact resistances is $R_{t}>R_{b}>R_{c}$. This result is consistent with the fact that the largest flux linkages occur between strands in different bundles of the cable and their contributions to the induced currents are inversely proportional to $R_{C}$.

The value of $R_{c}$, the contact resistance between strands in different bundles, could be smaller than the other contact resistances in the cable due to factors arising from cable compaction. Strands in different bundles are not as nearly parallel as other strand pairs when they come into contact and the concentration of stresses at the resultingly reduced contact area may reduce contact resistivity. However, the range of variation allowed for in Figure 2.14 is actually quite large (the scale is logarithmic, base 2) and yet the maximum induced currents only increase by a factor of approximately 6 at the very extremes of the domain. Since the induced currents in the baseline case are so small $(\sim 4 \mathrm{~mA})$, it can be concluded that variations in contact resistance would not significantly affect the stability of the cable.

\subsubsection{Current Distribution in Finite Length, Joint Terminated CICC}

Having solved for the self- and external-field effects in an infinitely long periodic cable, the effects of terminating a cable at a finite length in a low resistance "joint" will now be studied. As' will be seen, the results will differ dramatically from those previously calculated for two reasons which can loosely be termed "length effects" and "joint effects." 


\section{Length effects:}

If the cable length is not an integer multiple of the final twist pitch length (or half this length in the case of self-field studies) we will see effects of two "imbalances." An inductive imbalance will be caused because the strands are no longer fully transposed and thus the mutual-inductances are not equivalent for each strand in the cable (i.e., some strands are more tightly coupled to the other 26 strands than others). Also, the flux linkages through each mesh will no longer balance or cancel when summed over the length of the cable. A net flux linkage will exist which can be much larger than the individual mesh flux linkages.

At longer lengths, the effect of inductive imbalances is reduced since the relative differences between the mutual couplings of each strand are reduced. This will be demonstrated below. Thus, for long cables, non-periodicity is not too problematic from an inductive viewpoint. The net flux linkage, on the other hand, is not dependent on the total cable length but only on the amount by which the length differs from an integral multiple of the transposition length. Thus long cables see the same magnitude imbalances as short ones in terms of net flux linkage.

\section{Loint Effects:}

Once we talk about finite-length cables, we need to know something about how they are terminated. For magnet applications, the cable is always terminated in a low resistance joint to either the power supply lead or a second magnet hooked up in series. The subject of low resistance joints is a lengthy one but will not be discussed in any detail in this thesis. For background material, see Herbelot (1990) and references therein. For the purposes of this study, a joint will be idealized to be a section of cable in which of all of the strands are interconnected by resistors with the value $\mathrm{Rj}$. It is difficult to obtain an estimate of this idealized "transverse joint resistance" since all known joint measurements 
only give a value of the resistance across the joint, between the cable and the power supply lead, for instance.

Nonetheless, for the cables discussed here, it is safe to assume that the transverse resistance between the strands in the joint must be much less than the transverse resistance in the cable proper. This assumption is valid because the joints in question are specially designed to minimize contact resistance--the strands are stripped of the chrome-oxide layer and heat treated under pressure in the hopes they sinter together (Takayasu [1994]). The chrome-oxide on the strands within in the cable, on the other hand, gives them a relatively high contact resistance, as has been discussed above.

For the time being, the value of $\mathrm{Rj}$ will not be given and further results will retain $\mathrm{Rj}$ as a scaling factor. The only assumption made will be that $R \mathrm{j}<<R 1$. In the next chapter, a quantitative value for $\mathrm{Rj}$ will be inferred from experimental results based on a hypothesis which attempts to explain Ramp Rate Limitation.

\section{Time scales:}

Cables terminated in low resistance joints possess two distinct characteristic time constants. The first can be termed a "local time constant" and is similar to the time constants encountered in the periodic problem. The local time constant is governed by inductance differences on the scale of a single segment of cable and the contact resistance, $R 1$. This time constant is very small for the cables being considered here. The "overall time constant" is related to the inductance differences for the whole cable and the joint resistance, $\mathrm{Rj}$. Since the overall inductance differences are much greater than the local inductance differences and $\mathrm{Rj}$ is much smaller than $\mathrm{R} 1$, the overall time constant is much greater than the local time constant. 


\subsubsection{Self-Field, Steady-State Solution:}

In the initial stages of a transient, $t=0^{+}$, the current imbalances due to a ramping transport current are governed by local effects and are very similar to those of the periodic case. Almost instantaneously, however, at times greater than the local time constant, the generally increasing currents are governed by the overall mutual-inductances of the cabled strands, which may no longer be equal. Since the non-periodic case does not possess most of the symmetries used above to reduce the potential number of unknowns, it is not feasible to solve this problem for the entire time domain using the above techniques. However, since the time derivative of the currents quickly become dominated solely by whole length inductances and the strands can effectively be considered insulated on that same time scale, the current imbalance reaches a steady-state solution almost immediately. It is possible to solve for a steady-state distribution directly to find the correct solution for all but the very initial transients. The problem reduces to the simple circuit shown in Figure 2.15. Because the self- and mutual-inductances no longer balance (except in special cases), the cable will not relax to a uniform distribution.

Mathematical Model:

The key to the problem is an accurate calculation of the self- and mutualinductances between each strand. The model cable geometry described above is again used for this purpose with the cable length equal to an integer number of segments. For the finite length of cable, the coupling between each of the strand segments can again be calculated to give the complete set of mutual-inductances, $M_{i, n, j, m}$, as described above. The overall mutual-inductance between the full lengths of strands $i$ and $j$ is thus:

$$
M_{i, j}=\sum_{n} \sum_{m} M_{i, n, j, m}
$$




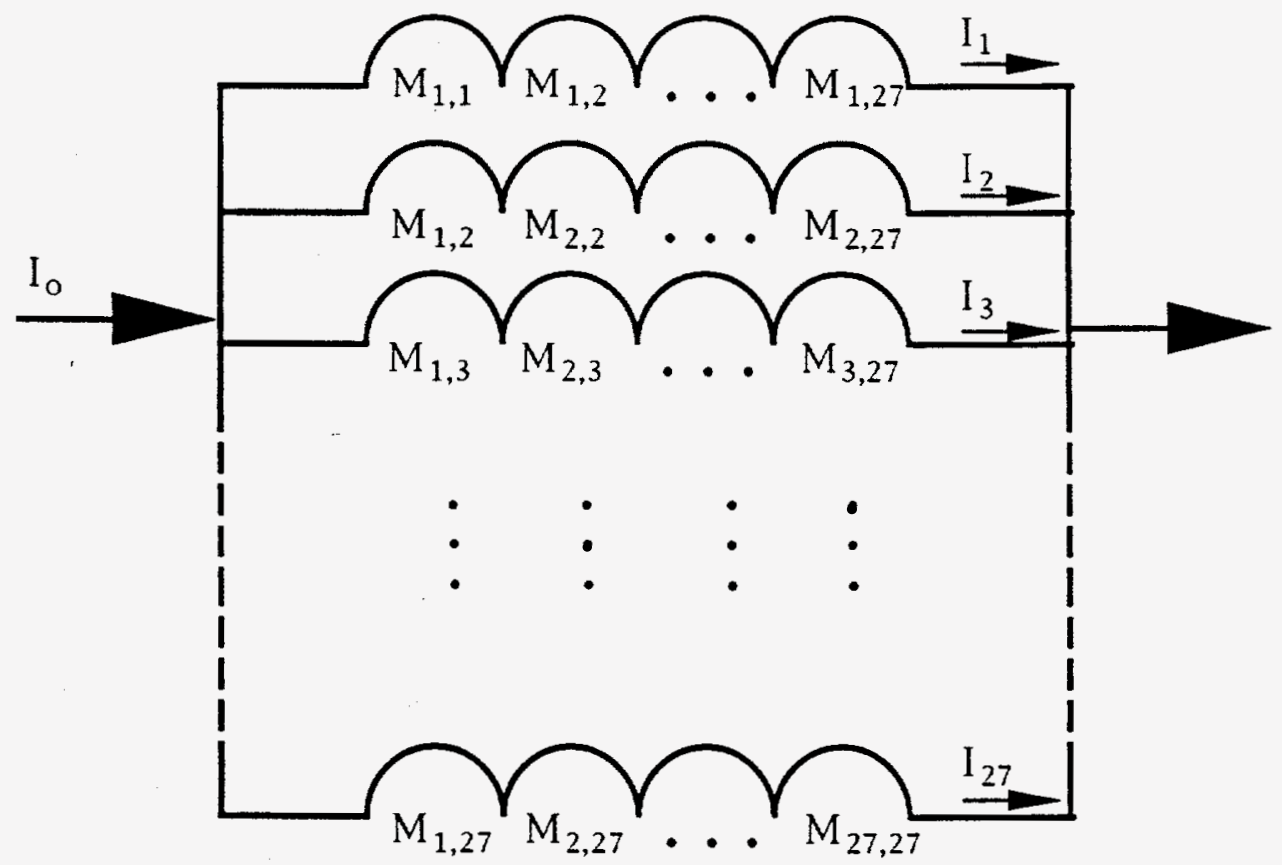

Figure 2.15 The self-field effect for linear ramping transport current in a finite length CICC can be approximated using a model composed of mutually coupled inductors joined in parallel.

where indices $m$ and $n$ cover the segment positions for the entire cable length. To give an idea of the scale of the calculated inductances for the length of cable used in the 27 strand experiments, the self-inductance of a strand was found to be approximately $1 \mu \mathrm{H}$.

The problem is thus reduced to the matrix equation:

$$
\mathbf{M} \cdot \dot{\mathbf{I}}=\dot{I}_{0} \cdot\{\mathbf{1}\}
$$

Where matrix $\mathbf{M}$ is comprised of elements $M_{i, j}$ and the vector $\dot{I}$ contains the time derivatives of the current in each strand, $\dot{I}_{i}$. $\dot{I}_{0}$ is again the ramp rate of the overall current and $\{1\}$ represents the unit vector. Since we are neglecting the effects of initial transients, the current in strand $i$ is merely: $I_{i}=t \dot{t}_{i}$, assuming the 
strands start with zero current and $t$ is time. Thus, for a ramping transport current, imbalances in the steady-state current derivatives are equivalent to the imbalances in the currents. After calculating the coefficients, solving the resulting equations is straightforward since the matrix is only $27 \times 27$ in size. The small size of the matrix allows for speedy solutions for cables of various lengths.

Results:

The steady-state current imbalances due to the self-field effect for a finite length of the 27 strand cable are shown in Figure 2.16 as a function of length. The currents are again normalized to the average current per strand and the length is given in units of a single segment length $(4.2 \mathrm{~mm})$. As is evident from the figure, for cables of more than a several twist pitches in length, (one twist pitch $=48$ segments) the current distribution due to self-field effect becomes nearly negligible. The rapid decay occurs because the inductances balance at every half multiple of the twist pitch length and thus it is only any "extra bit" of length

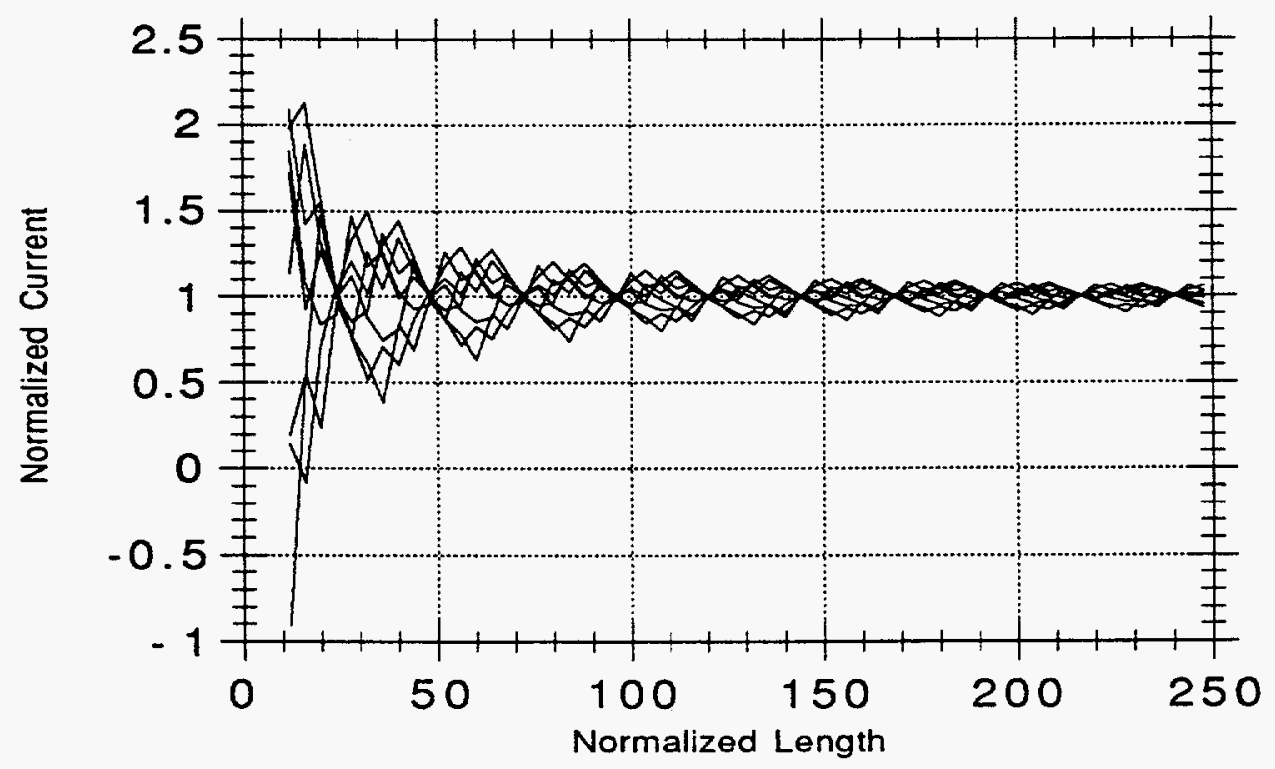

Figure 2.16 Current Imbalances vs. Normalized length of cable for linearly ramping transport currents. See text for details. 
beyond these points which contributes to the imbalance. At longer lengths, this imbalance is small compared to the overall inductance of the cable. For short cables, however, the effects can be pronounced and can adversely affect the current distribution in the cable.

If the pitch lengths are not exactly equal to their nominal values, there may not be any lengths of cable which would exhibit a true inductive balance. However, the overall magnitudes of the imbalances are not significantly greater than those calculated for the nominal case if the twist pitches are at least close to their nominal values. Although the geometric model used here breaks down for other than nominal twist pitches, the simpler model mentioned at the end of Section 2.2.3 can be used to approximate the inductances of the strands. (This model, based solely on twist pitches, was not used previously primarily because it does not model the inter-strand contacts as well as the model above.) Using Inductance formulas from Grover (1946) (see Appendix A), the self-inductance of each strand is found from Eq. (A.1) as if it were a straight wire and the mutualinductances are calculated from Eq. (A.2) as if the strands were straight and parallel. The separation distance is calculated from the simply twisting geometric model as the average distance between the strands over the given length. To establish a baseline, the current imbalance as a function of length for the nominal cable twist pitches using these approximations is shown in Figure 2.17. The current imbalance for a case where the twist pitches of the triplet, bundle, and cable are offset by 20,10, and 5 percent, respectively, is shown for the same lengths in Figure 2.18. In both figures, the currents are normalized to the average current per strand and the length is given as multiples of the nominal final twist pitch length, equal to $20.3 \mathrm{~cm}$. The figures show that the magnitude of imbalance due to self-field effect is relatively insensitive to small deviations from the nominal twist pitch lengths in the cable. 


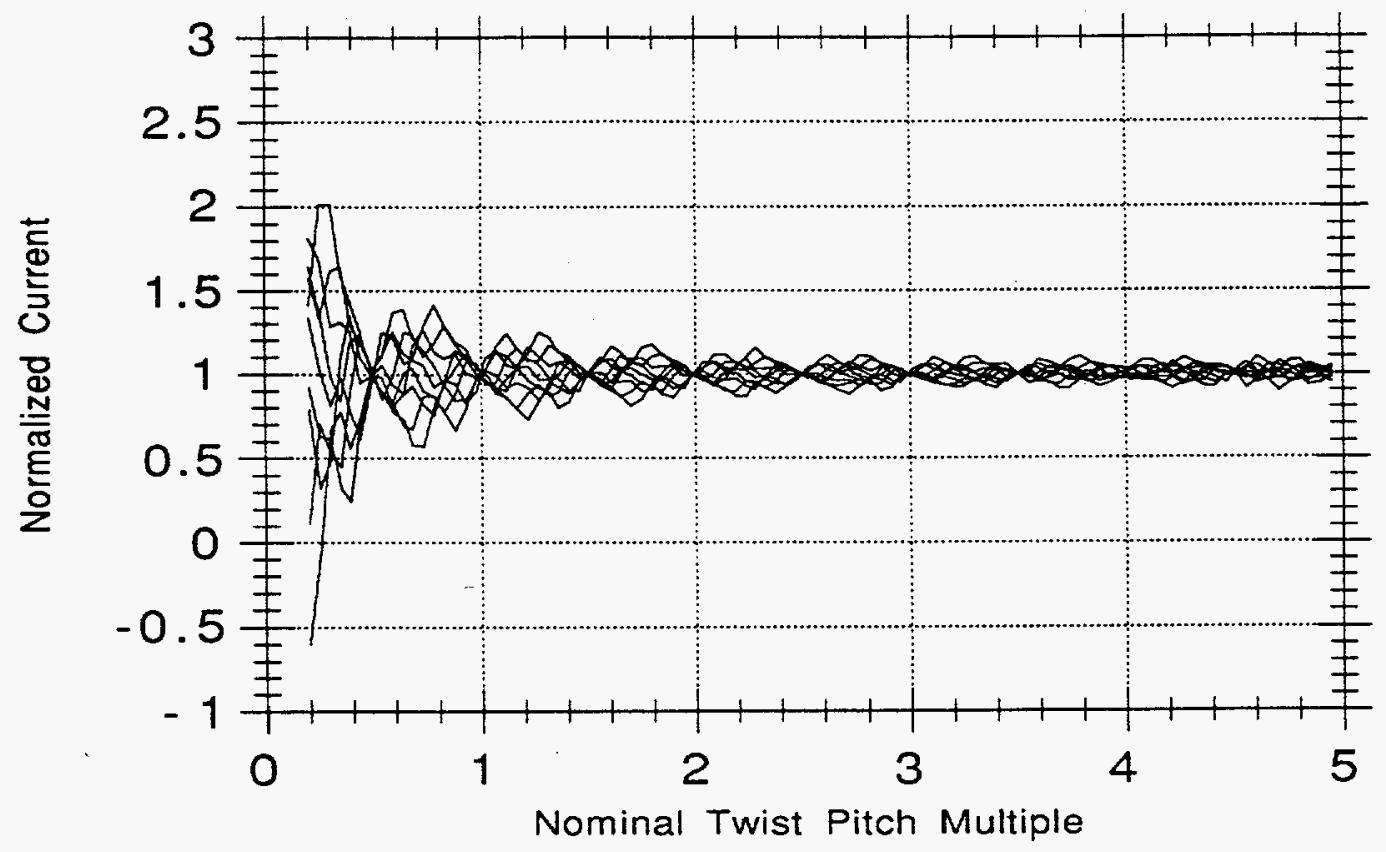

Figure 2.17 Current Imbalances vs. multiple of final twist pitch length for linearly ramping transport currents using a simplified geometric model. The twist pitches of each stage of cable are equal to their nominal values. The results should be compared to Figure 2.18 .

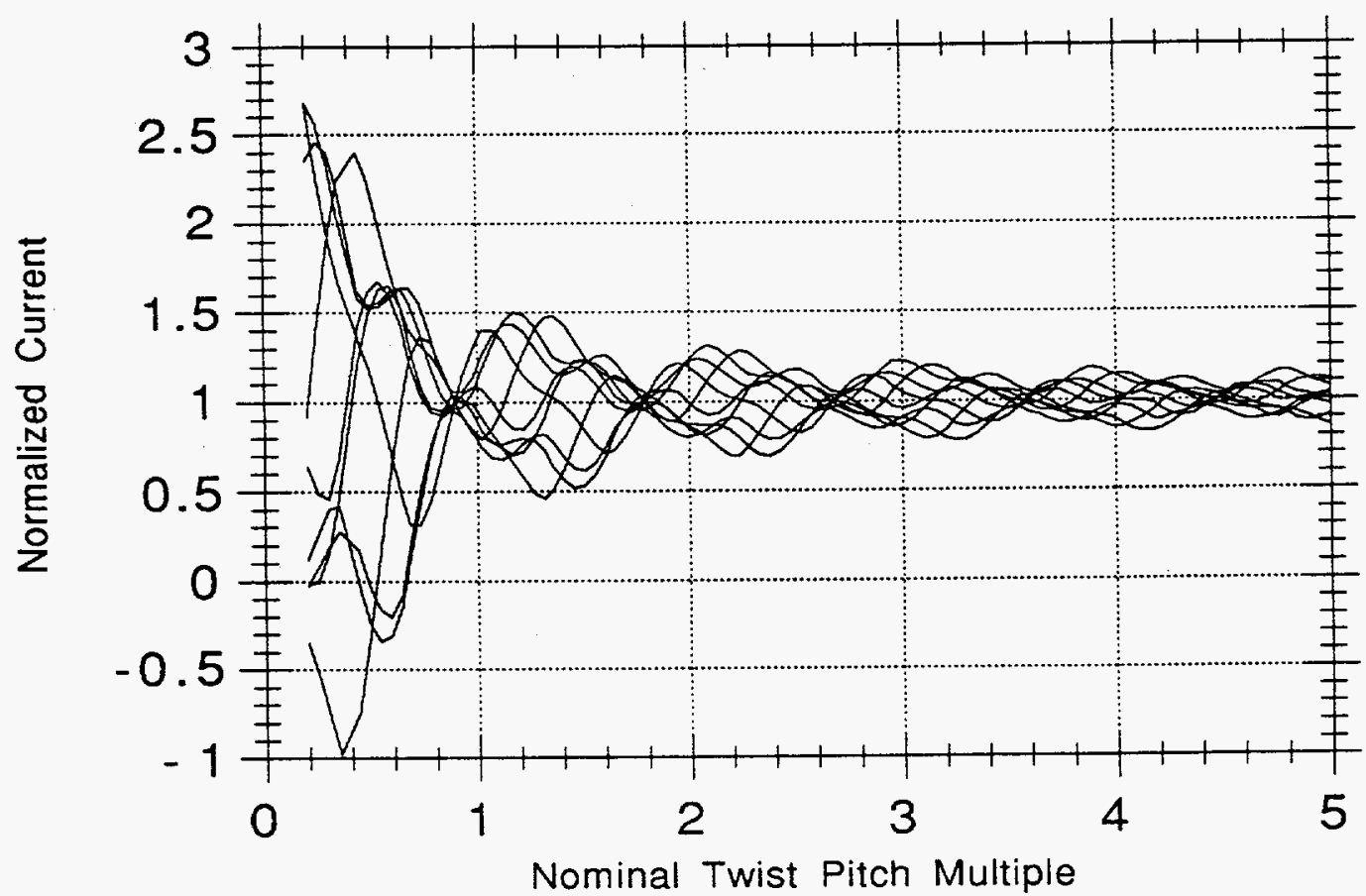

Figure 2.18 Current Imbalances vs. multiple of final twist pitch length for linearly ramping transport currents using a simplified geometric model. The twist pitches are $2-\%$ longer than the nominal value for the triplets, $10 \%$ longer for the bundles, and 5\% longer for the final stage The results should be compared to Figure 2.17 . 


\subsubsection{External-Field, Time-Dependent Solution:}

In an external-field, if a cable is not an integer multiple of twist pitch lengths, there is a net flux enclosed between the cabled strands even though they are twisted. While the length of the twist pitch puts a limit on the amount of net flux possible, the resultant current imbalances due to the external-field effect can still be significant. As the field changes, the change in net flux drives currents along entire lengths of strand in loops which connect through the joints. Since the joint resistance is very low and the net flux can be appreciable, these currents will be much larger than the currents found in the infinitely long periodic cable.

In the initial stages of ramping field, at times much less than the local time constant, the problem is similar to the periodic case described above. Local inductances dominate and the response develops on the time scale of the local time constant. At times on the order of the local time constant, though, the "local eddy currents" are dominated by the contact resistance and begin to saturate to values proportional to $\dot{B} / \mathrm{R} 1$. It would be difficult to solve for the time development of these initial transient responses numerically since we do not have any of the many "reductions" which allowed for the solution in the periodic case. However, the local eddy currents are small relative to the net eddy currents and thus can be neglected for the purposes of studying stability in cables of nonperiodic lengths.

At times slightly greater than the local time constant, the local eddy currents have stabilized, but the net eddy currents through the joints are still in the initial phases of their transient development. Since the eddy currents through the joint are governed by the whole length inductances, $M_{i, j}$, and the "transverse joint resistance", $R_{\mathfrak{j}}$, their time development is much slower than that of the local eddy currents, as has been explained above. For purposes of calculating the net eddy currents, the small amplitude local eddy currents can be 
neglected and the current in each strand can be considered to be zero at time $t=0$.

\section{Mathematical Model:}

The problem is greatly simplified by eliminating the local eddy currents. Each strand is considered to be a single branch with flux linkages and inductive couplings to each of the other strands. In the idealized joints at either end, each strand is also connected to each of the other strands by a resistance, $R_{\mathbf{j}}$. The problem is shown schematically in Figure 2.19 for a simplified case using only four strands. Since the two joints are considered to be identical, only the transverse currents in one need to be kept as independent variables. The number of connections between the 27 strands is $27^{*}(27-1) / 2=351$ giving, together with the 27 unknown strand currents, a total of 378 unknowns.

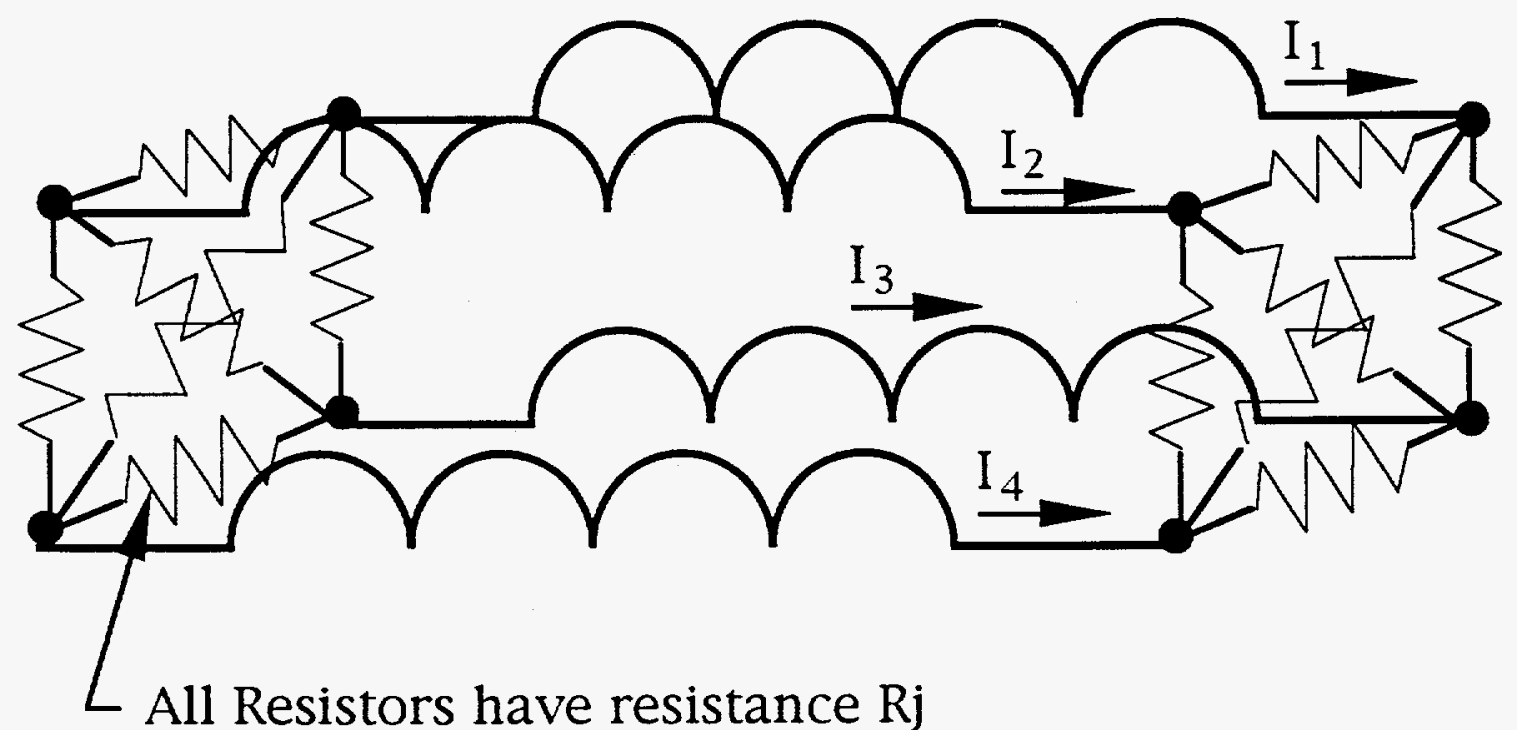

Figure 2.19 Idealized model of a four strand cable terminated in low resistance joints. Each strand is connected to the others at each joint through a resistance, $\mathrm{Rj}$. The strands are inductively coupled over their lengths but can otherwise be considered insulated. See text for further details. 
Kirchoff's Current Law is used at the 27 nodes in the idealized joint and Kirchoff's Voltage Law is applied around each of the 351 possible loops between strands running the length of the cable. The equations are thus:

$\mathrm{KCL}: \quad I_{i}+\sum_{j=i+1}^{27} i_{i, j}-\sum_{j=1}^{i-1} i_{i, j}=0 ; i=1 \ldots 27$

$\mathrm{KVL}: \quad 2 R_{j} i_{i, j}+\sum_{k=1}^{27}\left[M_{i, k} \dot{I}_{i}-M_{j, k} \dot{I}_{j}\right]=V_{i, j} ; i=1 \ldots 26 ; j=i+1 \ldots .27$

where the indices $i$ and $j$ refer to strand numbers; $i_{i, j}$ refers to the transverse current through the joint resistance between strands $i$ and $j ; I_{i}$ is the current through strand $i ; M_{i, j}$ is the whole length mutual-inductance between strand $i$ and $j$; and $V_{i, j}$ is the induced loop voltage due to the net flux trapped between strands $i$ and $j . M_{i, k}$ and $V_{i, j}$ are calculated using the strand configurations from Figure 2.2 for any length of cable equal to an integer multiple of the segment length. There is a factor of 2 in the resistance term of Eq. (2.25) to account for the joint resistance at both ends of the cable.

As before, the forward Euler finite difference scheme is used to transform the set of first order differential equations to a set of linear equations. Matrix techniques using iterative refinement are used to solve for the time development of the strand currents due to the net external flux changes for given lengths of cable. The procedure is similar to the transient analyses performed earlier for the periodic cases.

Results:

The problem is length dependent so it is difficult to show general results, however several trends can be noted. First, for cables which are finite in length but equal to an integer multiple of the transposition length, there are no net flux linkages and thus no net eddy currents. For cables of other lengths, the steady- 
state values of the net eddy currents are proportional to the difference between the actual length and the closest integer multiple of the period length. Thus, a cable of length equal to $p+1 / 2$ period lengths, where $p$ is an integer, will experience the largest possible steady-state eddy currents in a ramping field. The characteristic time constant for the transient development is monotonically dependent on length. Longer cables have higher inductances (but the same joint resistances) and thus longer overall time constants than shorter cables.

Quantitative results of the numerical model are shown in Figure 2.20 for the maximum strand currents found in 4 cables of different lengths: $24,72,120$, and 1224 segment lengths (approximately 10, 30, 50 and $515 \mathrm{~cm}$, respectively). These specific lengths were chosen because they represent "local maxima," equaling an integer $+1 / 2$ multiple of the final twist pitch. As expected, the steady-state values of the maximum eddy current is equal for the four cases, but the time constants increase with length. Because the value of the idealized joint resistance has not been assigned, the currents in the figure are normalized. The actual currents in amps would be: $I_{a c t}=I^{\prime} * \dot{B} /\left(1,000 * \mathrm{R}_{\mathrm{j}}\right)$, where $I$ is the dimensionless current in the figure. Similarly, the time is normalized such that the actual time in seconds is $t_{a c t}=t^{\prime} /\left(100,000^{*} R_{j}\right)$, where $t^{\prime}$ is the dimensionless time in the figure.

The fall-off in the amplitude of the steady-state net eddy currents as the cable length approaches an integer period multiple is shown in Figure 2.21. Again, only the current in one strand is shown, but the magnitude of all the other currents falls within this envelope. The same normalizations apply. Any integer multiple of 48 (the normalized period length) can be added to the abscissae without changing the results. Ideally, a cable would be designed to have a total length equal to a period length to totally eliminate the net flux and thus net eddy currents, but this would only work in the uniform field limit. In general, it 


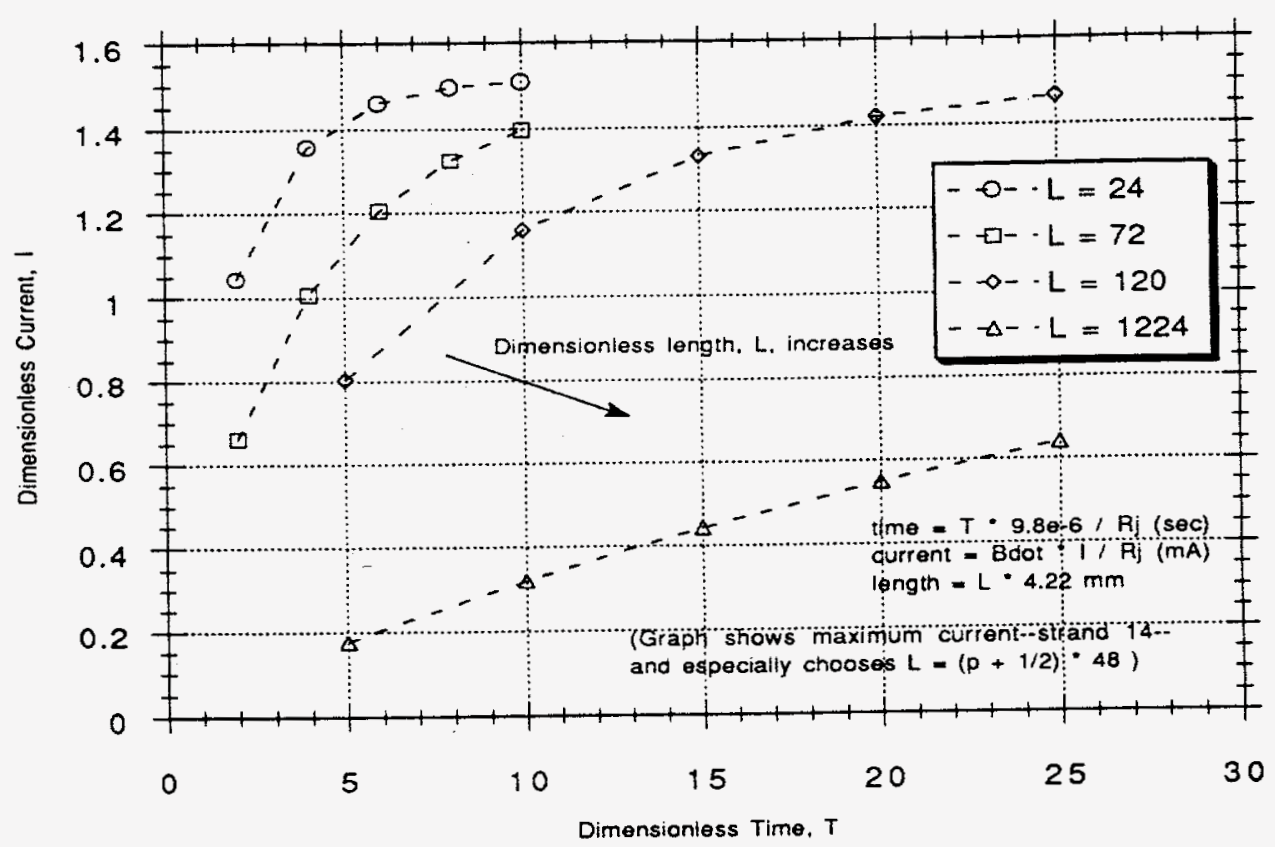

Figure 2.20 Dimensionless current vs. dimensionless time for the strand experiencing the maximum induced current due to a linearly ramping external magnetic field for cables of four dimensionless lengths: 24, 72, 120, and 1224 segment lengths. Each current approaches the same asymptotic value, but the time constant of each curve is length dependent.

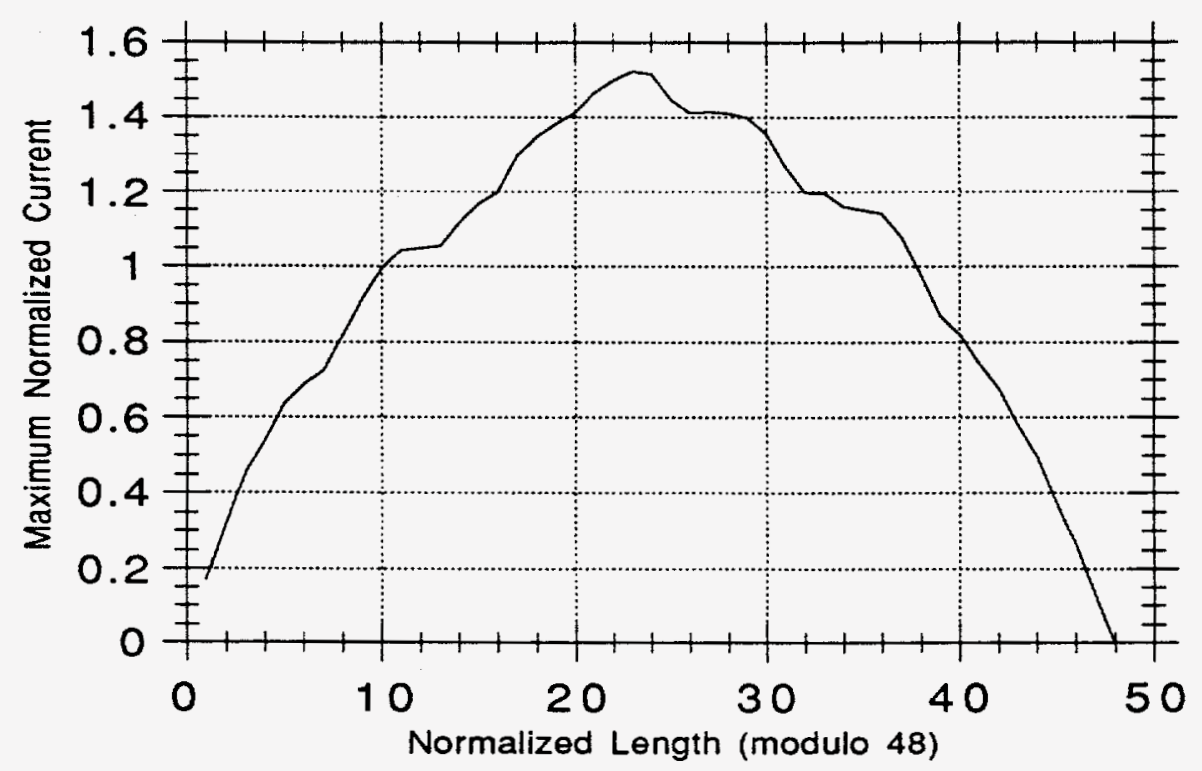

Figure 2.21 The maximum induced current in the cable is length dependent. At lengths approaching an integer multiple of the period length (48 segment lengths), the induced currents are minimal. At integer-plus-half multiples of the period length, the induced currents are maximized. 
would be nearly impossible to design a cable with no net flux linkage, but it is possible to limit the maximum possible net flux by keeping the twist pitches as tight as possible.

The steady-state currents in all of the strands for a cable equal to $p+1 / 2$ twist pitch lengths are shown as a histogram in Figure 2.22. Again the currents are normalized by $\dot{B} /\left(1,000^{*} R_{\mathbf{j}}\right)$. Without assigning a value to $R_{\mathbf{j}}$, however, it is impossible to compare the magnitude of the apparent imbalances with any benchmark as was possible in the periodic case. An experimental measurement which allows a direct approximation of $R_{j}$ would in this sense be very beneficial, but will have to wait for future work. In the next chapter, however, a value of $R_{j}$

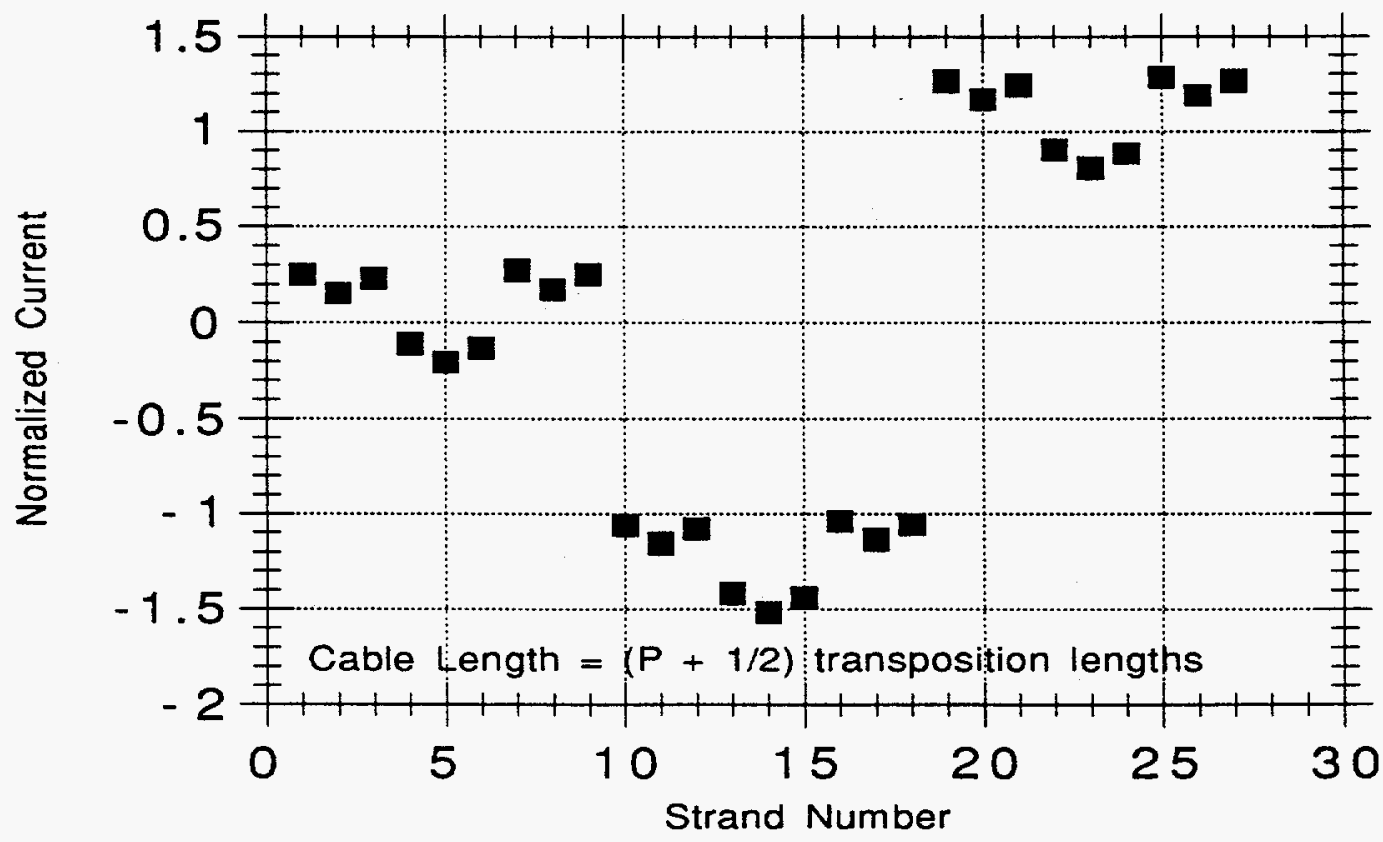

Figure 2.22 Histogram of the steady state currents induced by a linearly ramping magnetic field for each strand in the 27 strand sample for a cable length equal to an integer-plus-half multiple of the period length. 
will be inferred from existing experimental results but only after making several arguable assumptions.

\subsection{Summary}

In this chapter, the current distributions due to self- and external-field effects have been calculated for a 27 strand sample in both the infinite, periodic regime and the finite, joint terminated regime. The methods developed are equally applicable to other cable designs when the geometry can be idealized in a similar fashion as was used here. The results for the infinite periodic case could be given in absolute units since direct measurements of the transverse resistance between strands exist. For the joint terminated cases, the final results were normalized by the transverse resistance in the joint, a value which is as yet to be measured. Although each case presumed a starting point of zero field and zero current, solutions for differing initial conditions could be found in similar fashion.

\section{Infinite, Periodic Cases:}

In the infinite periodic case, the current distribution was found to be minor when compared with the operating currents typical of the ramp rate experiments examined. For the self-field scenario, the currents were found to equilibrate to a uniform distribution on time scales on the order of microseconds. Since typical ramp times are on the order of seconds, the self-field effect for fully transposed infinitely long periodic cables is seen to be negligible. Current imbalances for the external-field scenario also equilibrated very quickly, but not to a uniform distribution. The largest induced current for a $1 \mathrm{~T} / \mathrm{s} \mathrm{ramp}$, however, was only approximately $0.4 \mathrm{~mA}$. In any event, the imbalances encountered in the infinite, periodic case would not be large enough to affect the stability of the cable. The detail of the model does allow for estimates of AC 
losses due to self-and external-field effects, though, which would be an interesting subject for a future investigation with the difficult part of the analysis already complete.

Finite, Joint Terminated Cases:

For cables of finite length, important current imbalances due to ramping currents and fields can exist. These imbalances do not affect the $\mathrm{AC}$ losses of the cable, per se, because the transverse currents involved cause joule heating only in the joints." On the other hand, the induced currents may be large enough to affect the overall stability of the coil by increasing the total current in some strands above their critical current. Without knowing a value for the idealized transverse resistance in the joint, though, it is difficult to make quantitative predictions. Qualitatively, however, cables of short length will in general exhibit more current imbalances than longer cables since the self-field effects are potentially greater and the characteristic time constant for external-field effects will be shorter. This latter condition implies that any net eddy currents will be closer to their maximum saturated values at the end of a ramping event.

Despite the level of uncertainty in calculating current imbalances for finite-length cables, the next chapter will further examine their possible stability implications in hopes of explaining the Ramp Rate Limitation.

* Joint losses are generally considered to be a separate topic. 


\section{Chapter 3}

\section{Ramp Rate Stability in CICC Magnets}

\subsection{Overview}

The Ramp Rate Limitation exhibited by the US-DPC and the smaller scale simulation tests cannot be explained using the traditional stability analyses discussed in Chapter One. This chapter investigates the possibility that the nonuniform current distributions caused by the ramping field and transport current may be related to the observed limitation. Rather than decisively proving a correlation however, the conclusion is that the proposed instability mechanism is a viable one. In this light, the investigation presented in this chapter is not meant to comprise a full stability analysis but is instead intended to provide the background and motivation for more detailed study.

\subsection{Simplified Stability Model Based on Current Distribution for CICC}

Traditional stability models assume uniform magnetic field and current density distributions and study the effects of temperature perturbations. The stability model introduced here will assume uniform temperature and field distributions and will study the effect of current density "perturbations." A "stability event" will be defined in the model as a point where the total current in a strand, applied plus induced, reaches the strands limiting current value, as determined experimentally. At this point, the strand would begin joule heating and would most likely transfer current to neighboring strands. The cable's response to a stability event is thus a complicated one and answering whether or 
not a stability event would lead to quench is beyond the scope of this thesis.* Nonetheless, for the purposes of discussion, the modeled stability events will be compared with the observed Ramp Rate Limitation in the 27 strand experiment in the hopes of explaining general trends found in the data. The results of this experiment were presented in Figure 1.6. Since the cable is of finite length and is terminated in joints with resistances much lower than the contact resistance between the strands, the approximate solutions developed above for that scenario are applicable.

The model for determining a stability event can be given in terms of the total current in a single strand:

$$
\mathrm{I}_{\text {total }}=\mathrm{I}_{\mathrm{app}} / \mathrm{N}+\mathrm{I}_{\text {self }}+\mathrm{I}_{\text {ext }}=\mathrm{I}_{\text {critical }} / \mathrm{N}
$$

where $\mathrm{N}$ is the number of strands in the cable $\left(27\right.$, here); $\mathrm{I}_{\mathrm{app}}$ is the total transport current, $I_{\text {self }}$ is the amount of current imbalance in one strand due to self-field effects and $I_{\text {ext }}$ is the current imbalance due to external-field effects. $I_{\text {critical }}$ is defined as the maximum amount of current which could be carried by the cable assuming uniform current distribution. Since the temperature is considered to be constant, $\mathrm{I}_{\text {critical }}$ is only a function of the field in this model. In the experiment, the background magnetic field was fixed to be proportional to the applied current in order to simulate the operation of th full US-DPC magnet. The resulting "load-line" was:

$$
\mathrm{B}=1.58 \mathrm{I}_{\mathrm{app}}
$$

where $B$ is in $T$ and $I_{a p p}$ is in $k A$.

In the experiment, the maximum achievable current approaches an asymptote at longer ramp times which gives an experimental value for $\mathrm{I}_{\text {critical }}$ at full magnetic field $(\sim 8 \mathrm{~T}) . \mathrm{I}_{\text {critical }}$ at full field can be approximated from Figure

\footnotetext{
* Recent unpublished results from experiments on the 27 strand sample may indicate several "stability events" can take place in a single ramp without leading to quench but there is also evidence that a stability event does precipitate any eventual quench (Vysotsky, et. al. [1994]).
} 
1.6 to be $\sim 5 \mathrm{kA}$. The critical current at other magnetic fields of interest needs to be related to this value of Icritical at maximum field. For the range of experimentally determined quench currents at the various ramp rates, the associated magnetic fields (as determined by the load-line) are in the range of 6 $8 \mathrm{~T}$. Over this range of interest, the critical current density of the superconductor used in the strand, $\mathrm{Nb3Sn}$, decreases in an approximately linear fashion with the value at $8 \mathrm{~T}$ being approximately $70 \%$ of the value at $6 \mathrm{~T}$ (Bottura [1989]). Using this same ratio for the composite conductor, $\mathrm{I}_{\text {critical }}$ at $6 \mathrm{~T}$ can be approximated to be $7 \mathrm{kA}$ and the linear relation valid over the range of $6-8 \mathrm{~T}$ is roughly: $\mathrm{I}_{\text {critical }}=$ $13-B$, where $B$ is in $T$ and $I_{\text {critical }}$ is in $\mathrm{kA}$. Using the prescribed loadline, Eq. (3.2), this translates into a critical current for a single strand:

$$
\mathrm{I}_{\text {critical }} / \mathrm{N}=\left(13-1.58 \mathrm{I}_{\mathrm{app}}\right) / 27 \quad(\mathrm{kA})
$$

Where $I_{\text {app }}$ is given in $k A$.

The self-field imbalance for the 27 strand cable can be estimated from the analysis of Chapter Two. The overall length of the sample is 0.84 meters, equivalent to 200 segment lengths using the geometric model above. The current imbalance due to self-field effects for a cable of this length is shown as a histogram in Figure 3.1. The strand carrying the maximum current due to selffield imbalance has approximately 1.08 times the average current, thus $I_{\text {self, }}$, the amount of imbalance, has a maximum of $0.08 \mathrm{I}_{\mathrm{app}} / \mathrm{N}$. For the purposes of this rough analysis however, this unbalance will be considered a secondary effect since $\mathrm{I}_{\text {self }} \ll \mathrm{I}_{\mathrm{app}} / \mathrm{N}$ and we will use:

$$
\mathrm{I}_{\text {self }} \approx 0
$$

The background field imbalance is dependent on the ramp rate. Of the total length of 200 segments, only the 72 segments $(30 \mathrm{~cm}$ ) which comprise the "single loop coil" experience a transverse external-field. Thus the length for calculating whole strand inductances is different from the length used for 


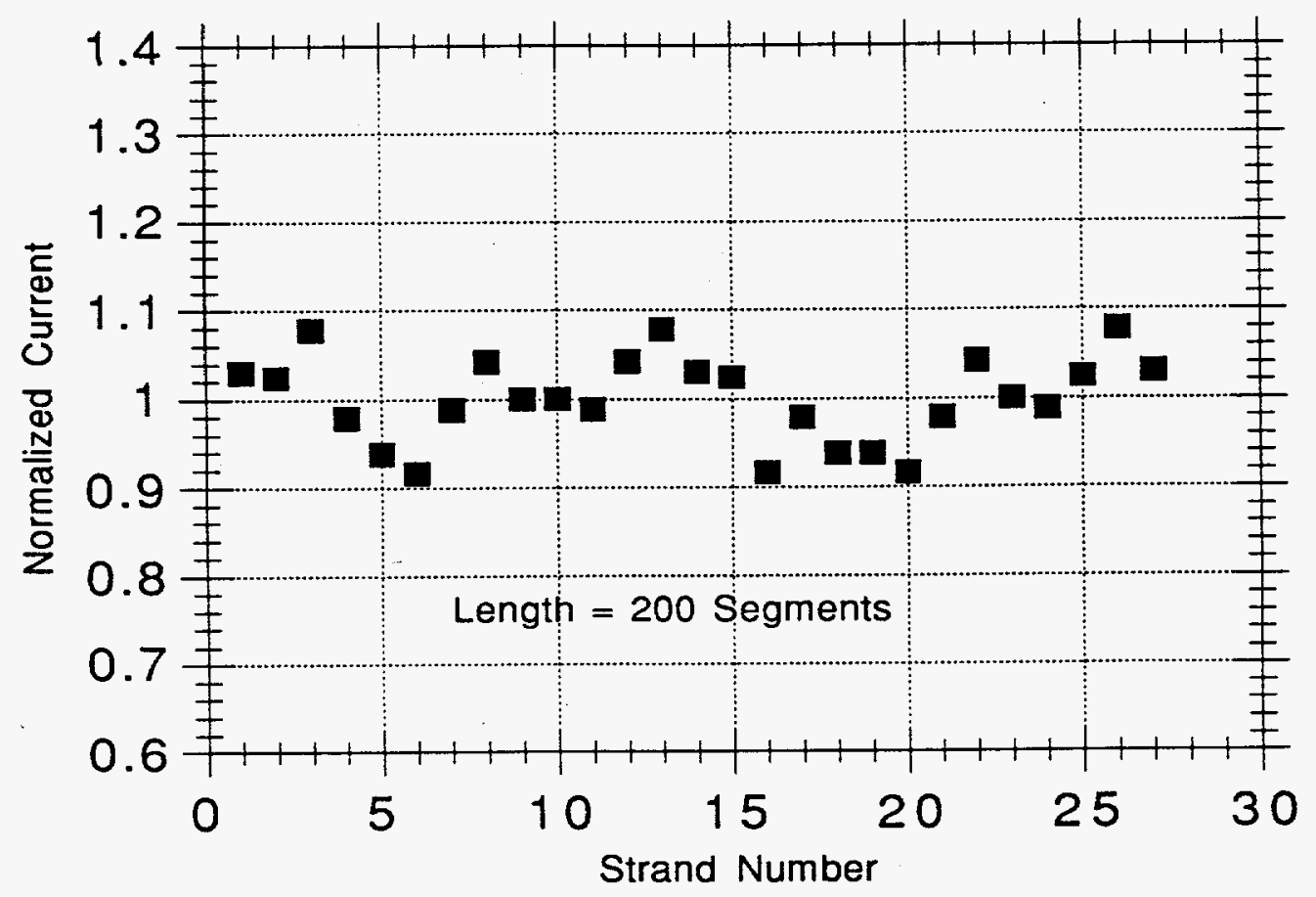

Figure 3.1 Histogram of the current imbalance due to a linearly ramping transport current for each strand in the 27 strand sample for a cable length equal to the length of the actual experimental cable, $0.84 \mathrm{~m}$ (200 segment lengths). Strands with a normalized current equal to unity are carrying $1 / 27$ th of the transport current.

calculating net external flux linkages. The result for the one strand which experiences the maximum induced current for such a cable is presented in Figure 3.2 as current vs. time. As in chapter two, the units are dimensionless. The curve is seen to have the form :

$$
I^{\prime}{ }_{\text {ext }}=C^{\prime}\left(1-\exp \left(-t^{\prime} / \tau^{\prime}\right)\right)
$$

where $I^{\prime}$ ext is the dimensionless current, $C^{\prime}$ is the dimensionless saturation value $\left(C^{\prime} \approx 1.5\right.$ for the "worst" strand case shown here), $t$ ' is the dimensionless time, and $\tau^{\prime}$ is the dimensionless time constant. Using a curve-fit of the numerical results, $\tau^{\prime} \approx 2.0$ and the resulting line is shown in the figure. Accounting for the scaling factors used, the actual current in $\mathrm{kA}$ would be $\mathrm{I}_{\text {ext }}=\mathrm{I}^{\prime}$ ext ${ }^{*} \dot{B} /\left(10^{6} \mathrm{R}_{\mathrm{j}}\right)$. The actual time, $t$, and time constant, $\tau$, in seconds would be the dimensionless values multiplied by $10^{-5} / R_{\mathfrak{j}}$. 


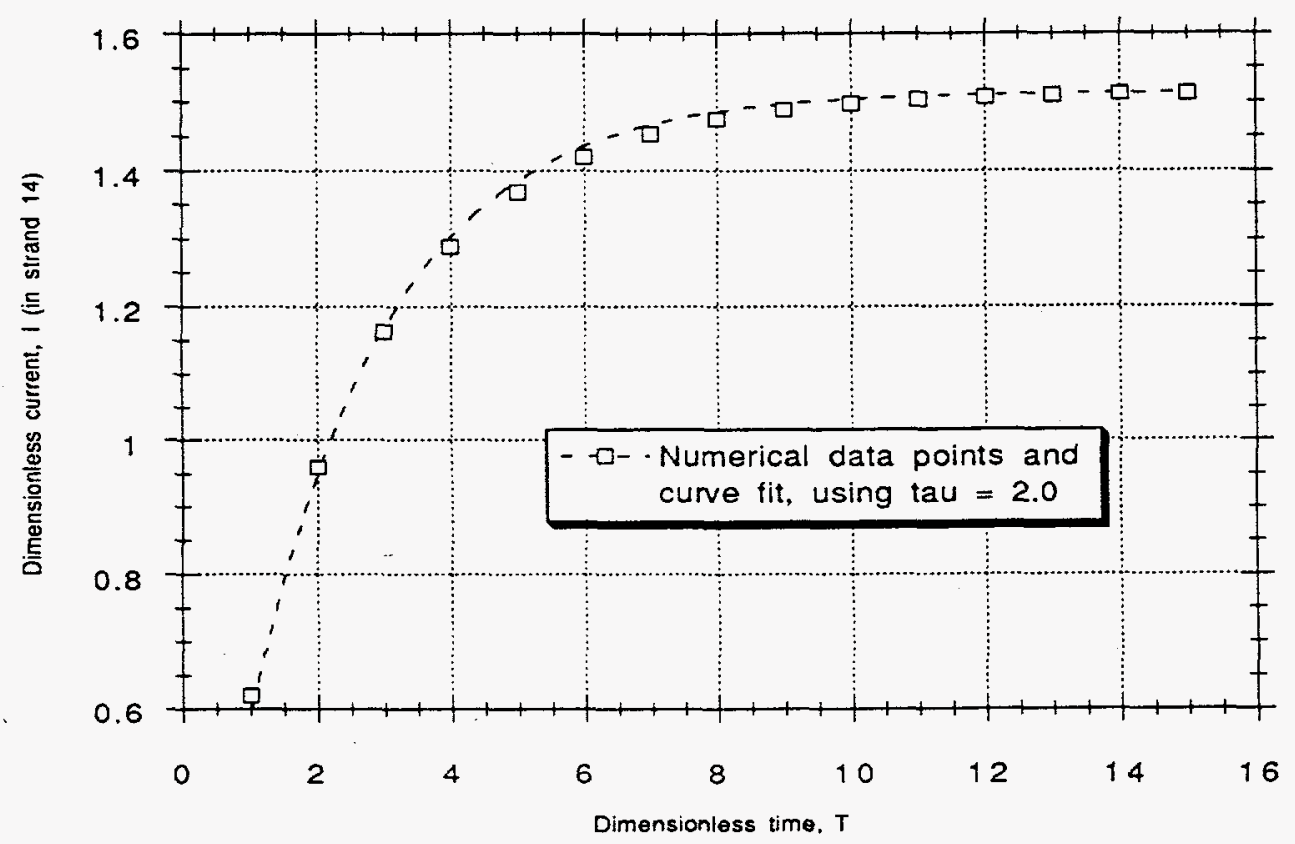

Figure 3.2 The maximum induced current in the 27 strand sample due to a linearly ramping external magnetic field vs. time. Both current and time are dimensionless. The total sample length is $0.84 \mathrm{~m}$ ( 200 segment lengths) with $0.30 \mathrm{~m}$ ( 72 segment lengths) experiencing a transverse magnetic field. The dimensionless time constant of the exponential time-development is found to be tau $=2.0$, giving the dashedline curve-fit shown.

Using Eq. (3.2) to relate the field change to the overall current ramp rate, $\dot{I}_{o}=I_{\text {app }} / t_{\text {ramp, }}$ we get the relation for $I_{\text {ext }}$ at any time, $t:$

$$
\mathrm{I}_{\text {ext }}=1.58 \cdot 10^{-6} C^{\prime} \frac{I_{\text {app }}}{t_{\text {ramp }}} \frac{\left(1-\exp \left(-10^{5} R_{j} \mathrm{t} / 2.0\right)\right)}{R_{j}} \quad(\mathrm{kA})
$$

where $t$ and $t_{\text {ramp }}$ are in seconds, $I_{a p p}$ is in $k A$ and $R_{j}$ is in $\Omega$.. An estimate of the total current in the "worst" strand, with $C^{\prime} \approx 1.5$, using equations (3.1), (3.4), and (3.6) is thus:

$$
\text { Itotal } \approx \mathrm{I}_{\text {app }} / 27+2.4 \cdot 10^{-6} \frac{\mathrm{I}_{\text {app }}}{\mathrm{t}_{\mathrm{ramp}}} \frac{\left(1-\exp \left(-10^{5} R_{\mathrm{j}} \mathrm{t} / 2.0\right)\right)}{R_{j}}(\mathrm{kA})
$$

A "stability event" will occur when this value is equal to the strands critical current. If a single stability event were to lead to a quench of the whole cable, the 
Ramp Rate Limitation would be found when the strand current just equals its

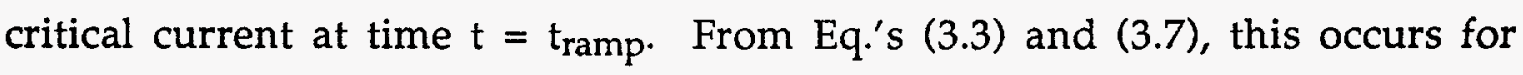
currents:

$$
\mathrm{I}_{\text {quench }}=13\left(2.58+6.5 \cdot 10^{-5} \frac{1-\exp \left(-10^{5} R_{j} \mathrm{t}_{\mathrm{ramp}} / 2.0\right)}{\mathrm{t}_{\mathrm{ramp}} R_{j}}\right)^{-1} \quad(\mathrm{kA})
$$

The only unknown parameter is $R_{j}$, in $\Omega$. The ramp time is given in seconds. The factor of $2.0 \times 10^{-5} / R_{j}$ in the exponent is the natural time constant of this particular cable, as it was found above. $I_{\text {quench }}$ vs. tramp is plotted against the experimental data in Figure 3.3 using a value of $\mathrm{Rj}=2 \times 10^{-5} \Omega$, found by curvefitting Equation (3.8) to the quenched points (solid circles) in the experimental data. The trend predicted by Equation (3.8) is seen to match the experimental results nicely and the fitting parameter needed to match the data seems reasonable; it is at least consistent with the original assumption that $\mathrm{Rj}<<\mathrm{R} 1$.

Until $\mathrm{Rj}$ can be measured independently, though, the most specific conclusion which can be reached is that the occurrence of "stability events" due to current imbalances in the cable is a plausible but not proven explanation for Ramp Rate Limitation. Further work is needed to confirm or refute this theory.

One factor in favor of the theory, however, can be now be mentioned. In separate experiments (Takayasu, et al. [1992]) on the 27 strand cable where the transport current was held constant while the background field was independently ramped, a ramp rate limitation very similar to the one found for simultaneous ramping of current and field was found. When the current was ramped and field held constant, however, only a much weaker ramp rate limitation was discovered. These different results are consistent with the predictions made here: the current unbalances and resulting stability events were 


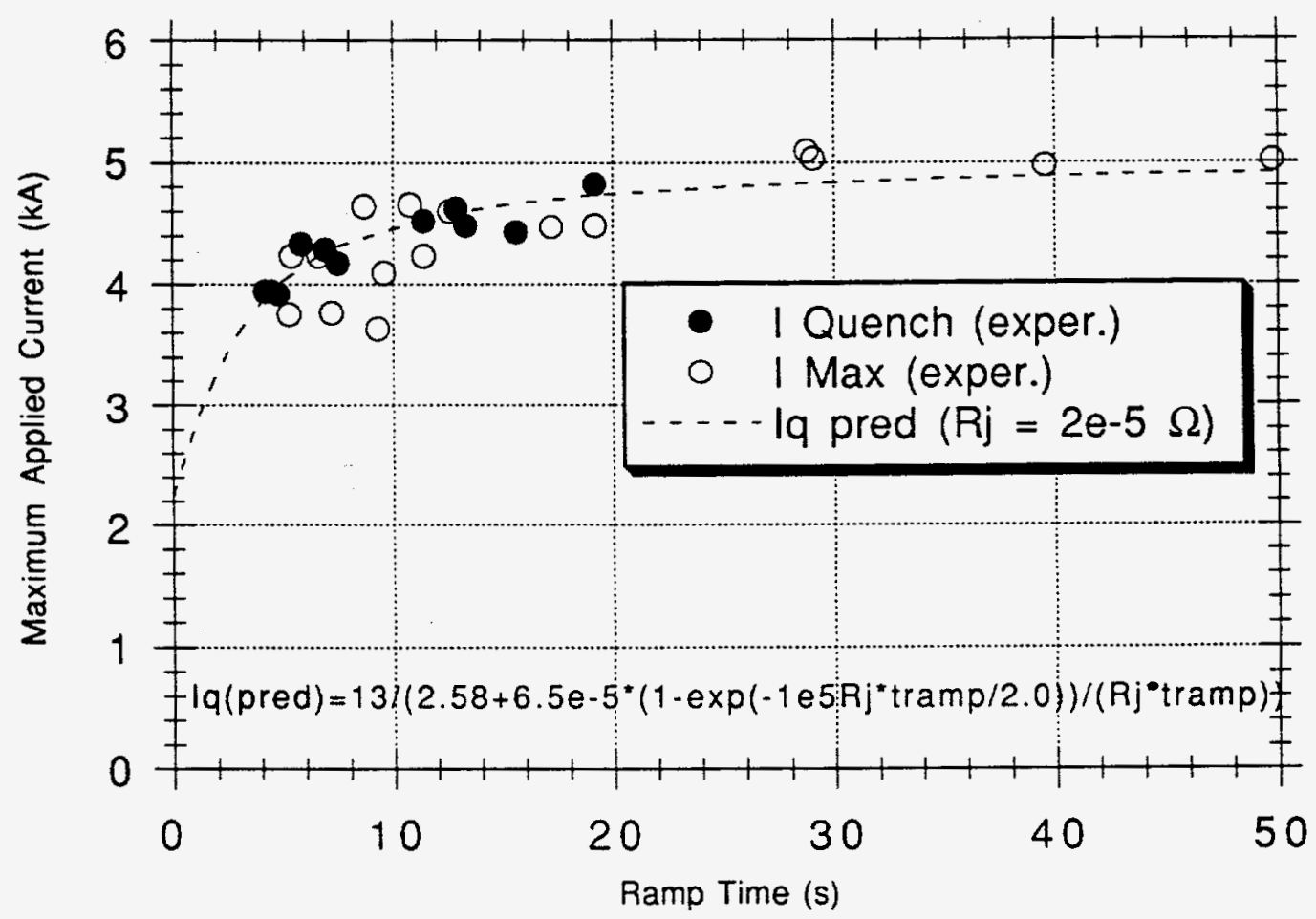

Figure 3.3 Measured data from Ramp-Rate Limitation tests on a 27 strand CICC. Solid circles $(\bullet)$ represent points at which the cable quenched during a linear ramp to the quench current in time, tramp. Open circles $\left(^{\circ}\right)$ represent currents which were reached without quench for ramp time, tramp. (Takayasu, et al., [1992])

strongly dependent on external-field effects while the self-field effects caused by ramping transport current were considered to be of only secondary importance.

It is also interesting to note that the model predicts a finite quench current for vanishingly small ramp times. This result is consistent with the results of the full-size US-DPC test shown in Figure 1.5 and the general finding that the Ramp Rate Limitation does not occur for currents below a certain "transition current" (Takayasu, et al. [1992]). This transition current for the Ramp Rate Limitation has previously been associated, however, with the limiting current separating the "ill-" and "well-cooled" regimes defined from traditional CICC stability analyses 
discussed above (Bottura, et al. [1991]). The transition current identified from this new analysis, based on Eq.'s (3.1), (3.6), and (3.8) in the limit $t=t_{\text {ramp }} \rightarrow 0$, is :

$$
I_{\text {trans }}=\frac{\tau R_{j}}{2.58+27 \cdot 1.58 \cdot 10^{-6} C^{\prime}} \quad(\mathrm{kA})
$$

which takes into account the linearization of critical current, the loadline, and the number of strands. The parameters which are model dependent, $\tau, R_{j}$, and $C^{\prime}$, are left as variables but in fact the factor of $R_{j}$ cancels out since $\tau$ is inversely proportional to $R_{\mathfrak{j}}$. Equation (3.9) thus says the transition current is dependent on the whole length inductive imbalances in the cable (proportional to $\tau$ ) and the net external flux linkage in the cable (proportional to $C^{\prime}$ ). The result is independent of cooling regime. A comparison of measured transition currents with Eq. (3.9) and with the traditionally defined transition current might be a way to investigate the true nature of Ramp Rate Limitation, at least for very fast ramp rates.

\section{3 Summary}

A correlation between Ramp Rate Limitation and current distribution in a cable has been demonstrated for the 27 strand US-DPC simulation ramp rate experiment. The validity of the correlation is limited by the uncertainty of the transverse joint resistance, $R_{j}$, as modeled in Chapter Two. A better joint model accompanied with accurate joint resistance estimates would be needed to confirm the usefulness of the model for predicting Ramp Rate Limitation. Also, the concepts of "stability event" for a single strand and the resulting heat generation would have to be developed further before it can be directly related to a full quench phenomenon in the cable. An improved model which merges the current-based stability analysis introduced here with traditional energy-based 
stability analysis might be needed to give a complete description of Ramp Rate Limitation.

Nonetheless the agreement of the simplified stability limit, Eq. (3.8), with experimental results is apparent and matches several measured trends: foremost, the calculated curve matches the data for the proper choice of scaling parameter; second, the model correctly predicts the strong dependence on $\dot{B}$ and only a weaker dependence on $\dot{I}$; and third, the model predicts a finite transition current below which no ramp rate limit occurs. Thus, although the model is basic, the results are encouraging and invite a more detailed analysis. 



\section{Chapter 4}

\section{Conclusions}

The effects of linearly-ramping transport currents and external magnetic fields on the current distribution in Cable-in-Conduit Conductors (CICC) has been studied for certain scenarios. A numerical model of the 27 strand cable used to simulate the US-DPC coil was used for all calculations, although the methods used can be equally applied to other multiply-twisted cables. The results of the calculations were compared to experimental measurements of the Ramp Rate Limitation phenomenon.

For cables which can be considered infinitely long and periodic in nature, the current unbalances due to self- and external-field effects were found to be negligible. Because such a cable is fully transposed, the transient imbalances due to the self-field effect vanish on the time scale of micro-seconds for the approximate values of transverse resistance found from measurements. Thus, even though the initial imbalances are significant, the stability of the cable is not affected since the total current in the cable is no more than a few milli-Amps during this transient response. Imbalances due to external-field effects do not vanish in time, but instead reach a steady-state in a time scale again on the order of micro-seconds. The maximum steady-state current imbalance is estimated to be on the order of milli-Amps for the ramping external-fields encountered experimentally. Compared to operating currents on the order of 100 's of Amps, this imbalance is again seen to be inconsequential with respect to the stability of the cable.

The detailed modeling presented for the infinitely long, periodic cable failed to discover any conditions which might affect the stability of the cable. 
However, the model does provide a tool for studying the AC losses associated with the inter-strand coupling for time-varying transport currents and magnetic fields. This second application of the model is potentially more useful than the current distribution study for which it was intended, but is not investigated in this thesis.

For finite cable lengths which were terminated in low resistive joints, certain simplifying assumptions were needed to solve for the current distribution in linearly-ramping magnet fields and transport currents. Resultingly, only the dominant currents due to "whole-length" effects were found while the relatively small currents due to "local effects" similar to those in the periodic case were ignored. It was necessary to use a simplified model of the inter-strand contacts within the joint and the calculated results could only be found to within a factor of $R_{j}$ the characteristic resistance between strands in the joint.

Self-field effects for finite cables can be significant since the cable is no longer fully transposed, in general. However, the current imbalances due to the self-field effect were seen to diminish with increasing length, and thus are of primary concern only for shorter cables. The effects of joint resistance were not included in the analysis of this case and thus the presented results are only valid for the initial transient response, but the general trend of decreasing imbalances for increasing lengths is still valid.

The external-field effect is not related to overall length but instead to differences between the cable length and the transposition length. The net flux linkage between entire lengths of strands--connected through the joints-- is maximized for cable lengths equal to integer-plus-half multiples of the transposition length. Since the joint resistances can be very small, the eddy currents driven by changes in the net linked flux can be large. Quantitative results cannot be directly presented since the joint resistance, $R_{j}$, is an unknown 
parameter. In general though, the maximum induced eddy current is related to the length of the cable (as explained above) and is proportional to the rate of change of the magnetic field and inversely proportional to the joint resistance. The maximum current is approached asymptotically on a time scale which is proportional to the total length of the cable and inversely proportional to the joint resistance.

The experimental results found for the 27 strand US-DPC simulation tests were studied using the newly developed models for current distribution due to self- and external-field effects in finite-length cables. By assuming that the encountered Ramp Rate Limitation was due to current imbalances in the cable, a new stability model was introduced which defined the quench current in terms of the maximum current carried by the most "overloaded" strand. The resulting relation (Eq. 3.8) matches the measured data when the characteristic joint resistance is chosen to be: $\mathrm{Rj}=2 \times 10^{-5} \Omega$, The comparison was shown in Figure 3.3.. (N.B. the value $R_{j}$ should not be compared to any "joint resistance" published in the literature since it is defined in a much different manner.)

While the oversimplified mathematical model of the joints and the lack of a measured value for the parameter $R_{j}$ eliminate the possibility of making authoritative conclusions at this time, the simplified model seems to exhibit the same trends as do the measured data: the calculated values reasonably agree with experimental values; the predicted asymptotic behavior at longer ramp times as well as the finite limiting current at very fast ramp times are both consistent with measurements and current conjecture; and finally, both the model and experiments show a strong dependence on the rate of change of the external magnetic field with only a weak dependence on the rate of transport current ramping. 
The general agreement of the simplified stability analysis presented here, based solely on current distribution in the cable, with the experimentally measured Ramp Rate Limitation is encouraging. The effects of current distribution due to ramping magnetic fields should be considered in future stability analyses. A more detailed model which includes thermal effects as well as electrodynamical effects may finally offer a complete explanation of Ramp Rate Limitation. 


\section{Appendix A}

\section{Inductance Formulas}

The following formulas were taken from Grover (1946):

Self-Inductance of a Round Straight Conductor:

$$
L=0.002 l\left[\ln \frac{2 l}{\rho}-\frac{3}{4}\right]
$$

where $l$ is the length of the conductor in $\mathrm{cm}$. and $\rho$ is the radius of the conductor in $\mathrm{cm}$. The self-inductance, $L$, is in micro-Henries.

Mutual-Inductance of Two Round, Parallel, Overlapping Conductors of Equal Length:

$$
M=0.002 l\left[\ln \left(\frac{l}{d}+\sqrt{1+\frac{l^{2}}{d^{2}}}\right)-\sqrt{1+\frac{d^{2}}{l^{2}}}+\frac{d}{l}\right]
$$

where both conductors are of length $l$, in $\mathrm{cm}$. and $d$ is the distance of separation between the longitudinal axes, in $\mathrm{cm}$. The mutual-inductance, $\mathrm{M}$, is in microHenries.

Mutual-Inductance of Two Round, Parallel, Non-Overlapping Conductors of Equal Length:

$$
M=\frac{1}{2}\left[\left(M_{2 l+\delta}+M_{\delta}\right)-2 M_{l+\delta}\right]
$$

where both conductors are of length $l$, in $\mathrm{cm}$. and $\delta$ is the axial component of the distance between the nearest endpoints. $M_{x}$ represents the mutual-inductance of two parallel overlapping inductors of equal length, $x$, (Equation A.3) with the distance of separation $d$ of the given problem.

Mutual-Inductance of Two Round, Parallel, Non-Overlapping Conductors with a Common Endpoint:

$$
M=\frac{1}{2}\left[M_{2 l}-2 M_{l}\right]
$$

where both conductors are of length $l$, in $\mathrm{cm}$. and $M_{x}$ represents the mutualinductance of two parallel overlapping inductors of equal length, $x$, (Equation A.3) with the distance of separation $d$ of the given problem. 


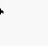

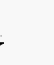




\section{Bibliography}

- Amemiya, N., et al., (1992) "Current Distribution in Strand and Instabilities in AC Superconducting Cables," ICFA Workshop on AC Superconductivity (ASCS92), Tsukuba, Japan.

- Bottura, L., (1989) "General use Properties Package (FORTRAN)," The Net Team, Garching, Germany.

- Bottura, L., et al. (1991) "Design Criteria for Stability in Cable-in-Conduit Conductors," Cryogenics 31, 510.

- Bottura, L., Minervini, J. V., (1987) FORTRAN code: HESTAB, The NET Team, Garching, Germany.

- Bottura, L., Minervini, J. V., (1991) 'Modeling of Dual Stability in a Cable-inConduit Conductor," IEEE Trans. Mag. 27, 1901.

- Bromberg, L., et al., (1993) "Anomalous Behavior in ICCS," 15th IEEE SOFE Conference, Hyannis, MA.

- Bruzzone, P., (1992) "Coupling Current Losses in Transversal Field," NET Team report No. N/B/3500/33/A, Garching, Germany.

- Carr, W. J., (1983) AC Loss and Macroscopic Theory of Superconductors, Gordon and Breach, New York.

- Egorov, S. A. et al. (1992) "Interstrand Coupling AC-Losses in Multistage Cable-in-Conduit Superconductors," Cryogenics 32 Supp, 439.

- Grover, F.W. (1946) Inductance Calculations, Dover Press, N.Y.

- Haus, H. A., Melcher, J. R., Electromagnetic Fields and Energy, Prentice Hall, Princeton, N.J.

- Herbelot, O., (1990) "Superconducting Cable Joint Resistance," S. M. Thesis, Nuclear Engineering Department, MIT, Cambridge, MA.

- Hoenig, M. O., (1980) "Internally Cooled Cable Superconductors, Parts I and II," Cryogenics, July and August 1980.

- Hoenig, M. O., Iwasa, Y., Montgomery, D. B.,(1975) Proc. 5th Int. Conf. Magnet Technology, ed. N. Sacchetti, M. Spadoni, and S. Stipcich, pub. Laoratori Nazionalis del CNEN, Frascati Italy, p. 20. 
- IMSL inc., (1987) FORTRAN Subroutines for Mathematical Applications, Houston, TX.

- Kawabata, S. et al. (1994) "Interstrand Coupling Effect on Losses and Current Distributions in Superconducting Cable Conductors," Cryogenics 39, No. 4.

- Knoopers, H.G., et al., (1985) "Distribution of Currents in a 6-strand Superconducting Cable," Magnet Technology Conference, Zurich.

- Mints, R. G., Rakhmanov, A. L., (1988) "The Current-Carrying Capacity of Twisted Multifilamentary Superconducting Composites," Journal of Physics D, 20, 826

- MTT Plama Fusion Center, (1991) "Proceedings of US-DPC Test Results Workshop," Cambridge, MA.

- Niessen, E., (1993) Continuum Electromagnetics of Composite Superconductors, CIP-Gegevens Kononklijke Bibliotheek, The Hague, Netherlands.

- Niessen, E.M.J, van Damme, R.M.J. (1993) "Influence of Stochastic Contact Resistances on Coupling Losses, Cryogenic Engineering Conference," Albuquerque, N.M.

- Orlando, T. P. (1991), Foundations of Applied Superconductivity AddisonWesley, Reading, Mass.

- Painter, T. A., et al., (1992) "Test Data from the US-Demonstration Poloidal Coil Experiment," MIT Plasma Fusion Center, Cambridge, MA.

- Press, W. H., et al. (1989), Numerical Recipes, Cambridge University Press, Cambridge.

- Steeves, M. M., Minervini, J. V., (1991) "Status of US-DPC Ramp Rate Limitation Analysis," Proceedings of the ITER Magnet R\&D Workshop, Sept. 23-27, Naka-machi, Ibaraki, Japan, pp. 137-152.

- Strang, G., (1986) Introduction to Applied Mathematics, Wellesley-Cambridge Press, Cambridge, MA.

- Takayasu, M., (1992) MEMO: "Strand-Strand Contact Resistance," MIT Plasma Fusion Center, Cambridge MA.

- Takayasu, M., (1994) private conversation, April 26, 1994. 
- Takayasu, M., et al. , (1992) "Measurements of Ramp-Rate Limitation of Cable-in Conduit Conductors," Applied Superconductivity Conference, Chicago.

- Torii, S., et al., (1993) "Analysis of Degradation in AC Superconducting Cables," IEEE Transactions on Applied Superconductivity, 3, No. 1.

- Turck, B., (1992) "Transverse Resistance in Conduit Conductors: AC Losses versus Current Transfer," Applied Superconductivity Conference, Chicago.

- Vysotski, V. (1993) presentation at MIT Plasma Fusion Center, October 21.

- Vysotsky, V., et al., (1994) MIT Plasma Fusion Center, work in progress.

- Wilson, M. N. (1983) Superconducting Magnets, Clarendon Press, Oxford. 